\title{
Mid-IR Variability and Dust Reverberation Mapping of Low- $z$ Quasars. I. Data, Methods, and Basic Results
}

\author{
Jianwei Lyu (吕建伟) (10), George H. Rieke (1), and Paul S. Smith \\ Steward Observatory, University of Arizona, 933 North Cherry Avenue, Tucson, AZ 85721, USA; jianwei@email.arizona.edu \\ Received 2019 July 31; revised 2019 September 22; accepted 2019 September 24; published 2019 November 15
}

\begin{abstract}
The continued operation of the Wide-field Infrared Survey Explorer (WISE), combined with several ground-based optical transient surveys (e.g., CRTS, ASAS-SN, and PTF), offers an unprecedented opportunity to explore the dust structures in luminous active galactic nuclei (AGNs). We use these data for a mid-IR dust reverberation mapping (RM) study of 87 archetypal Palomar-Green quasars at $z \lesssim 0.5$. To cope with various contaminations of the photometry data and the sparse time sampling of the light curves, procedures to combine these data sets and retrieve the dust RM signals have been developed. We find that $\sim 70 \%$ of the sample (with a completeness correction up to 95\%) has convincing mid-IR time lags in the WISE WI ( $3.4 \mu \mathrm{m})$ and $W 2(\sim 4.5 \mu \mathrm{m})$ bands, and they are proportional to the square root of the AGN luminosity. Combined with previous $K$-band $(\sim 2.2 \mu \mathrm{m}) \mathrm{RM}$ results in the literature, the inferred dust emission size ratios are $R_{K}: R_{W 1}: R_{W 2}=0.6: 1: 1.2$. Under simple assumptions, we put preliminary constraints on the projected dust surface density at these bands and reveal the possibly different torus structures among hot-dust-deficient, warm-dust-deficient, and normal quasars from the reverberation signals. With multi-epoch Spitzer data and later WISE photometry, we also explore AGN IR variability at 10-24 $\mathrm{m}$ over a $5 \mathrm{yr}$ timescale. Except for blazars and flat-spectrum radio sources, the majority of AGNs have typical variation amplitudes at $24 \mu \mathrm{m}$ of no more than $10 \%$ of that in the $W 1$ band, indicating that the dust reverberation signals damp out quickly at longer wavelengths. In particular, steep-spectrum radio quasars also lack strong $24 \mu \mathrm{m}$ variability, consistent with the unification picture of radio-loud AGNs.

Unified Astronomy Thesaurus concepts: Active galactic nuclei (16); Quasars (1319); Radio loud quasars (1349); Radio quiet quasars (1354); Infrared astronomy (786); Reverberation mapping (2019); Time series analysis (1916); Dust continuum emission (412); Extragalactic astronomy (506); Supermassive black holes (1663); Infrared galaxies (790)
\end{abstract}

Supporting material: figure set, machine-readable tables

\section{Introduction}

The circumnuclear dusty structures in active galactic nuclei (AGNs) bridge the gap between the black hole (BH) accretion disk and the host galaxy interstellar medium (ISM) and lay the foundation for AGN unification proposals (Antonucci 1993; Urry \& Padovani 1995; Netzer 2015). However, due to their complex geometry and small sizes, it is very challenging to characterize torus structures as well as the properties of the constituent dust grains.

Infrared (IR) reverberation mapping (RM) opens a window to peek inside the so-called AGN dusty torus. When the UV/ optical emission of the $\mathrm{BH}$ accretion disk changes, the varying signal travels at the speed of light to the torus, causing it to react as reprocessed emission in the IR but with a time lag. We can analyze the optical and IR light curves of AGNs to retrieve spatial information reflected in the response of the torus to changes in irradiation from the central engine. The procedure is similar to the RM originally proposed by Blandford \& McKee (1982) and widely applied to study the broad-line regions (BLRs) and accretion disks (e.g., Peterson et al. 2004). The time lag of the IR emission was originally reported for the Seyfert 1 galaxy Fairall 9 by Clavel et al. (1989) and soon after analyzed in terms of RM to constrain the AGN dusty circumnuclear structure by Barvainis (1992). The behavior was subsequently observed for a number of AGNs; the most noteworthy work is the systematic study of time lags between the near-IR ( $K$-band) and the optical of $\sim 30$ Seyfert 1 nuclei through ground-based monitoring. The results follow an
$R \propto L^{1 / 2}$ size-luminosity relation, as expected if there is a similar dust sublimation temperature in all sources (e.g., Oknyanskij \& Horne 2001; Suganuma et al. 2006; Lira et al. 2011; Koshida et al. 2014; Pozo Nuñez et al. 2014; Mandal et al. 2018; Ramolla et al. 2018).

Glass (2004) conducted a $J H K L(1.25-3.45 \mu \mathrm{m})$ long-term monitoring program of 41 Seyfert nuclei and reported tentative time-lag measurements between the $U(\sim 0.36 \mu \mathrm{m})$ and $L$ $(\sim 3.45 \mu \mathrm{m})$ bands for five objects. Given the stable and cool space environment, high-precision photometry was made possible by the IRAC instrument on Spitzer, and a mid-IR dust reverberation study was carried out at 3.6 and $4.5 \mu \mathrm{m}$ and reported for the reddened type-1 AGN in NGC 6418 (Vazquez et al. 2015). However, due to the limitations in observing at $3-5 \mu \mathrm{m}$ from the ground and the difficulty and cost of carrying out long-term coordinated ground- and space-based targeted observations, no other detailed reverberation studies in this wavelength range are available.

Now there is a new possibility to conduct systematic dust $\mathrm{RM}$ at 3-5 $\mu \mathrm{m}$ for bright AGNs with the continued operation of the Wide-field Infrared Survey Explorer (WISE). Launched in 2009 December, this satellite performed a mid-IR all-sky survey, was put into hibernation, and then reactivated as the Near-Earth Object WISE (NEOWISE) mission for asteroid hunting (Mainzer et al. 2014). The telescope completes an allsky map roughly every 6 months and had provided photometric data with 12-13 epochs that cover a time period of $8 \mathrm{yr}$ by the end of 2018. The AGNs in Seyfert galaxies studied by 
Koshida et al. (2014) had typical luminosities up to about $3 \times 10^{11} L_{\odot}$, and reverberation time delays at this luminosity are expected to be $\sim 100$ days, i.e., too short to be sampled well at the NEOWISE cadence. However, the cadence is satisfactory for more luminous AGNs. When combined with ground-based wide-field optical transient surveys, these mid-IR multi-epoch data can be used to explore the dust reverberation signals close to the spectral energy distribution (SED) peak of the AGN hot dust emission at $\sim 3 \mu \mathrm{m}$ for the first time. Given the depth, range, and sky coverage of these surveys, this approach has the potential to draw general conclusions about the structure of a typical AGN and the surrounding material in a statistically meaningful way. In this paper, we will demonstrate how to make the best use of these public data sets and present the results from an RM analysis at $3-5 \mu \mathrm{m}$ of $87 z<0.5$ PalomarGreen (PG) quasars.

Despite the presence of terrestrial atmospheric windows at 10 and $20 \mu \mathrm{m}$, few studies of AGN IR variability are available there because of the sensitivity limitations of ground-based telescopes in the thermal IR. Nonetheless, studies of individual blazars have found large-amplitude coordinated variations from the visible through $10 \mu \mathrm{m}$ (e.g., Rieke \& Kinman 1974). Neugebauer \& Matthews (1999) reported a broader-based study based on coordinated data at $J(1.27 \mu \mathrm{m}), H(1.65 \mu \mathrm{m})$, $K(2.23 \mu \mathrm{m}), L^{\prime}(3.69 \mu \mathrm{m})$, and $N(10.6 \mu \mathrm{m})$ of $25 \mathrm{PG}$ quasars over several decades. They concluded that the blazar 3C 273 (PG 1226+023) was the only source that clearly varied at the $N$ band, and another radio-quiet quasar, PG 1535+547, might also be variable in this band, since its light curve mimicked the pattern seen at the shorter wavelengths. With the data collected for a large sample of AGNs repeatedly observed by Spitzer and WISE, we will provide an updated study of AGN variability behavior at 12 and $24 \mu \mathrm{m}$ over a timescale of $4-5 \mathrm{yr}$ and establish its relation with IR variability at shorter wavelengths.

This paper is organized as follows. We describe the data and the compilation of multiband light curves in Section 2. To retrieve the dust reverberation signals rigorously, Section 3 introduces a new method for the cross-correlation analysis between the low-cadence optical and mid-IR light curves. We present the results from the $3-5 \mu \mathrm{m}$ dust RM analysis, as well as the 10-24 $\mu \mathrm{m}$ variability study, in Section 4. Discussion about the origin of AGN mid-IR variability, the circumnuclear dust structure, and its relation to the BLR can be found in Section 5. Section 6 is the final summary.

Some technical details are left to appendices. Appendix A evaluates the quality of the photometric survey data used. We provide the derivations that relate time lag to torus size in Appendix B. In Appendix C, we reexamine the Seyfert 1 sample whose variability in the $K$ band was studied by Koshida et al. (2014).

Throughout this paper, we adopt the cosmology $\Omega_{m}=0.27$, $\Omega_{\Lambda}=0.73$, and $H_{0}=71 \mathrm{~km} \mathrm{~s}^{-1} \mathrm{Mpc}^{-1}$.

\section{Photometric Data and Light Curves}

\subsection{Data Ensemble}

The primary sample used for our mid-IR dust reverberation study is all 87 PG quasars at $z<0.5$ (Schmidt \& Green 1983; Boroson \& Green 1992). For the $24 \mu \mathrm{m}$ variability study, we also included 33 members of this PG sample plus another 106 quasars within the same redshift range to increase the statistical significance. Table 1 and Figure 1 provide an overview of the time-series data sets that have been used in this work. We present the details below.

\subsubsection{Optical Data from Ground-based Transient Surveys}

We collected optical photometry of the PG quasars from the Catalina Real-Time Transient Survey (CRTS; Drake et al. 2009). This program utilizes two Steward Observatory telescopes, a $1.5 \mathrm{~m}$ telescope on Mt. Lemmon, Arizona, and a $0.68 \mathrm{~m}$ telescope on Mt. Bigelow, Arizona. Both survey the northern sky, covering timescales from minutes to years. All images are unfiltered and processed using the SExtractor package with the standard aperture photometry. With a sequence of $30 \mathrm{~s}$ exposures, the $1.5 \mathrm{~m}$ can reach as faint as $m_{V} \sim 21.5$, while the $0.68 \mathrm{~m}$ limit is $m_{V} \sim 19$. Photometry for sources brighter than $V=13$ can be problematic due to source saturation and nonlinearity of the response. We gathered the 2005-2013 photometry values from the public archive of the second data release. According to Graham et al. (2017), the published error model of the CRTS archival photometry is problematic, and we follow their methodology to make the necessary corrections to these data.

We also utilized the $V$-band optical data from the All-Sky Automated Survey for Supernovae (ASAS-SN; Shappee et al. 2014), which are publicly available at the ASAS-SN lightcurve servers (Kochanek et al. 2017). The data were obtained through a global network of $2014 \mathrm{~cm}$ diameter telescopes (camera lenses) with commercial-level CCD cameras that have observed nearly the entire sky continuously since 2012. The image FWHM is $\sim 16^{\prime \prime}$ with an $\sim 8^{\prime \prime}$ pixel size. The photometry is measured with a 2 pixel radius aperture, and the background is estimated with a 7 pixel radius annulus. Typical photometric uncertainties are less than $0.075 \mathrm{mag}$. The calibration is done by observing nearby APASS stars. Typically, ASAS-SN photometry saturates at 10-11 mag, and the depth is roughly $m_{V} \sim 17$ under good weather conditions.

Finally, we gathered the optical time-sequence photometry for the PG quasars from the Palomar Transient Factory (PTF; Law et al. 2009) Data Release 3 (DR3), which includes all data collected during the survey from 2009 March to 2012 December and some selected data obtained from 2013 to 2015 January. The PTF data nicely fill the time gap between the CRTS and ASAS-SN light curves. This survey was done with the Palomar 48 inch $(1.2 \mathrm{~m})$ Samuel Oschin Telescope with a typical 2!"0 FWHM imaging resolution. The photometry was taken mostly through two broadband filters, SDSS$g$ ' and Mould- $R$, reaching $4 \sigma$ limiting $\mathrm{AB}$ magnitudes of $m_{g^{\prime}} \sim 21.3$ and $m_{R} \sim 20.6$ in $60 \mathrm{~s}$ exposures. Both bands saturate at $\sim 14 \mathrm{mag}$.

Since PG $1226+023$ (also known as 3C 273) is saturated in CRTS and PTF, we have looked for alternative data sets to build its optical light curve. In fact, this object had been systematically monitored from late 2008 to the middle of 2018 as part of the Steward Observatory (SO) spectropolarmetric monitoring project (Smith 2016). ${ }^{1}$ These observations were carried out with the SPOL CCD spectropolarimeter (Schmidt et al. 1992) at either the SO $2.3 \mathrm{~m}$ Bok Telescope on Kitt Peak or the SO $1.54 \mathrm{~m}$ Kuiper Telescope on Mt. Bigelow. By convolving the spectra with a standard Johnson $V$-band filter, $\sim 300$ photometric measurements with high signal-to-noise ratio $(\mathrm{S} / \mathrm{N})$ covering $10 \mathrm{yr}$ are available. We also collected the

1 http://james.as.arizona.edu/ psmith/Fermi/ 
Table 1

Time-series Data Sets Used in This Work

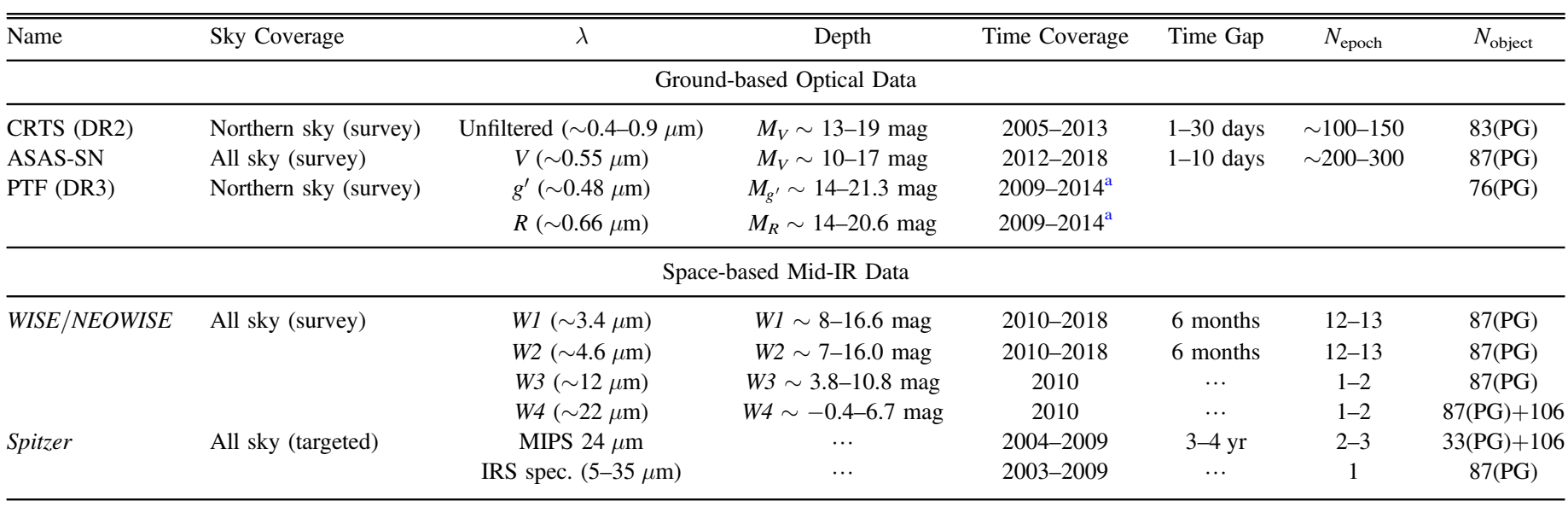

Note.

${ }^{a}$ Only selected $g$ - and $R$-band data are available after 2013 January 1 (through 2015 January 28 ) in this data release.

$V$-band photometry of 3C 273 with the Light Curve Generator (LCG) on the AAVSO website, ${ }^{2}$ which archives photometry from various facilities of bright variable objects. We omitted measurements flagged as being discrepant or having measurement uncertainties $>0.1 \mathrm{mag}$. The AAVSO data provide an optical light curve for 3C 273 from 2005 to 2019.

\subsubsection{Multi-epoch Mid-IR Photometry from WISE and NEOWISE}

The WISE mission performed an all-sky survey in four bands at 3.4, 4.6, 12, and $22 \mu \mathrm{m}$ from 2010 January through 2010 October. Then the telescope carried out a 4 month NEOWISE program using the first two bands without cryogen before going into hibernation in 2011 February. In 2013 December, WISE was reactivated and began the post-hibernation survey (the NEOWISE reactivation mission, or NEOWISE-R). WISE/NEOWISE observes the entire sky roughly every 6 months. All of the single-epoch images have been processed, and profile-fit photometry of each detection has been carried out by a dedicated pipeline. ${ }^{3}$

We gathered all of the single-epoch profile-fit photometry measurements in the $W 1(\sim 3.4 \mu \mathrm{m})$ and $W 2(\sim 4.6 \mu \mathrm{m})$ bands from the WISE and NEOWISE missions, up to the NEOWISE 2019 Data Release, from the NASA/IPAC Infrared Science Archive. Typically, these data cover a time period from 2010 January to 2018 December with a 3 yr gap between 2011 and 2014. Each object was observed for 12-13 epochs, with 10-20 exposures acquired within each epoch. To construct the mid-IR light curves, we only used detections from good-quality frame sets, following the suggestions given in the online documentation. ${ }^{4}$

\subsubsection{0-25 $\mu \mathrm{m}$ Measurements from Spitzer and WISE}

To test for variability at $\lambda>20 \mu \mathrm{m}$, we obtained MIPS $24 \mu \mathrm{m}$ measurements (PID 40053 and PID 50099; PI: George Rieke) of a heterogeneous sample of quasars that had been

\footnotetext{
2 https://www.aavso.org/lcg

3 See details in http://wise2.ipac.caltech.edu/docs/release/allwise/expsup/ and http://wise2.ipac.caltech.edu/docs/release/neowise/expsup/.

4 That is, frame quality score qual_frame $>0$, frame image quality score qi_fact $>0$, South Atlantic Anomaly separation saa_sep $>0$, and Moon masking flag moon_masked $=0$; see http://wise2.ipac.caltech.edu/docs release/neowise/expsup/sec2_3.html.
}

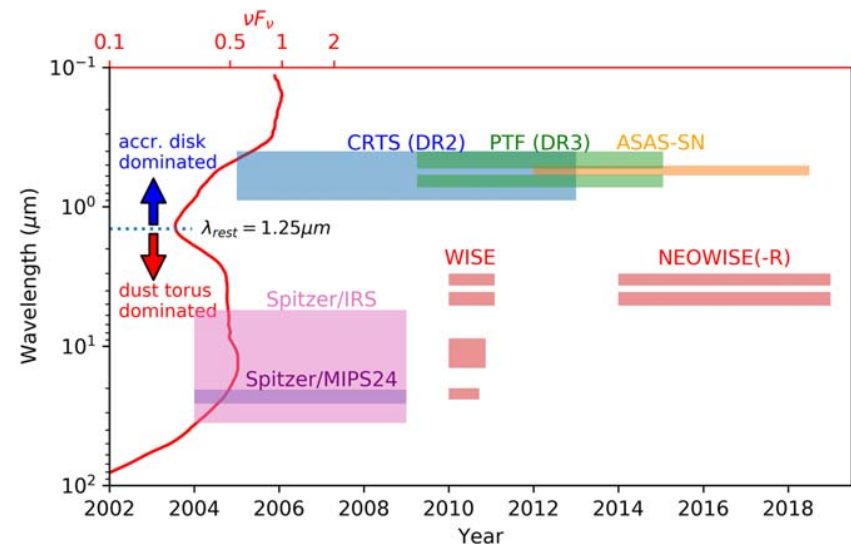

Figure 1. Time coverage and wavelength sampling of the different time-series data sets used in this work. We also show the intrinsic SED shape of the normal AGN template at $z=0.15$ (mean redshift of the PG sample) along the $Y$-axis and highlight the separation between the UV-optical bump and the IR bump with dotted blue line.

measured at the same wavelength by Spitzer for various previous observing programs. All of the observations were conducted during the Spitzer cryogenic mission from 2004 to 2009 and sample a time interval of 3-4 yr. The entire sample was observed twice, while a subset of nearly 60 objects was observed during a third epoch roughly $1 \mathrm{yr}$ after the secondepoch observation. To best utilize the instrumental stability and minimize subtle possible systematic effects, these AGNs were observed in exactly the same manner for all epochs. In this way, systematic effects associated with different durations for data collection events (DCEs) or a different total number of exposures are eliminated. In all cases, flux density measurements of the AGNs at $24 \mu \mathrm{m}$ were obtained using the standard MIPS photometry-mode astronomical observation template (AOT) with either 3 or $10 \mathrm{~s}$ DCEs.

All observations were processed using the standard reduction algorithms of the MIPS Data Analysis Tool (DAT) with the same processing steps and calibrations on all targets for both observations (Gordon et al. 2005). This included subsampling the detector array pixels by a factor of 2 to produce final mosaicked images of $363 \times 401$ pixels $^{2}(\sim 7 ! 5 \times 8$ ! 3$)$. Aperture photometry using the DAOPHOT package (Stetson 1987) within 
Table 2

Quasar Flux Measurements at $24 \mu \mathrm{m}$ and the Variability Results

\begin{tabular}{|c|c|c|c|c|c|c|}
\hline Name & $z$ & $\begin{array}{c}\text { JD } \\
-2,450,000\end{array}$ & $\begin{array}{l}\mathrm{f} 24 \\
\mathrm{mJy}\end{array}$ & $\begin{array}{c}\text { Error } \\
\text { mJy }\end{array}$ & $\begin{array}{c}\text { Stdev } \\
\text { of Change }\end{array}$ & Comments \\
\hline \multirow[t]{3}{*}{ 2MASX J00070361+1554240 } & 0.114 & $3,194.8$ & 63.5 & 0.7 & & RQ \\
\hline & & $4,676.2$ & 62.9 & 0.7 & 0.6 & \\
\hline & & $5,376^{\mathrm{a}}$ & 63.3 & 2.6 & 0.2 & \\
\hline \multirow[t]{3}{*}{ PG $0026+129$} & 0.142 & $3,747.4$ & 43.2 & 0.7 & & RQ \\
\hline & & $4,677.8$ & 43.2 & 0.7 & 0.0 & \\
\hline & & 5,382 & 44.1 & 3.8 & 0.2 & \\
\hline \multirow[t]{3}{*}{ 2MASS J00300421-2842259 } & 0.278 & $3,550.2$ & 125.1 & 1.0 & & RQ, IRAS F00275-2859 \\
\hline & & $4,675.4$ & 124.7 & 1.0 & 0.3 & \\
\hline & & 5,362 & 122.7 & 3.9 & 0.5 & \\
\hline \multirow{3}{*}{ 2MASS J00411870+2816408 } & 0.194 & $3,195.3$ & 74.5 & 0.8 & & RQ \\
\hline & & $4,514.7$ & 73.6 & 0.8 & 0.9 & \\
\hline & & 5,392 & 76.6 & 2.5 & 1.2 & \\
\hline \multirow[t]{3}{*}{ 2MASX J00505570+2933281 } & 0.136 & $3,218.3$ & 58.0 & 0.6 & & \\
\hline & & $4,514.7$ & 57.3 & 0.7 & 0.8 & \\
\hline & & 5,394 & 57.3 & 2.3 & 0.0 & \\
\hline \multirow[t]{3}{*}{ PG $0052+251$} & 0.154 & $3,217.8$ & 68.7 & 0.7 & & $\mathrm{RQ}, W 4$ high due to strong $18 \mu \mathrm{m}$ feature \\
\hline & & $4,509.2$ & 68.9 & 0.7 & 0.2 & \\
\hline & & 5,393 & 76.0 & 2.4 & 2.9 & \\
\hline$\ldots$ & $\ldots$ & $\ldots$ & $\ldots$ & $\ldots$ & $\ldots$ & $\cdots$ \\
\hline
\end{tabular}

Notes.

${ }^{\mathrm{a}}$ For the midpoint of the WISE observations.

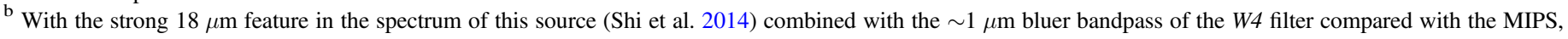
one can account for the higher signal seen by WISE.

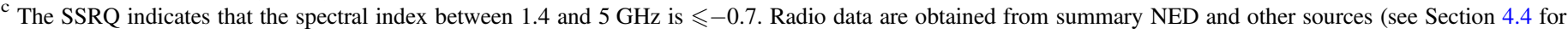
details).

${ }^{\mathrm{d}}$ Blazars are identified from Mao et al. (2016) and additional sources; see the text.

e The FSRQ indicates that the spectral index between 1.4 and $5 \mathrm{GHz}$ is $>-0.7$.

f This source is radio-intermediate by our criteria but radio-loud using others (Laor et al. 2019); given its flat spectrum also, we classify it as radio-loud.

(This table is available in its entirety in machine-readable form.)

IRAF was performed on the calibrated mosaicked $24 \mu \mathrm{m}$ images. The default parameters of the aperture photometry include an aperture of 24 pixels $\left(\sim 30^{\prime \prime}\right)$ and a sky annulus for background subtraction of $60-90^{\prime \prime}$ from the center of the photometric aperture. In a few cases, smaller apertures were used to avoid contamination by nearby field objects. Flux densities were calculated using an aperture correction of 1.105 and a conversion factor of $0.0454 \mathrm{MJy} \mathrm{sr}^{-1}\left(\mathrm{DN} \mathrm{s}^{-1}\right)^{-1}$ (Engelbracht et al. 2007). No color corrections have been applied to the photometric results.

Because the reductions are identical for all measurements of each object, the relative brightness is determined very accurately; i.e., for repeated measurements of standard stars, MIPS achieved repeatability of $\sim 0.4 \%$ (Engelbracht et al. 2007). Because the quasars are much fainter than the stars and hence less immune to faint background structures, we ascribe a systematic error of $0.7 \%$ (see Section 4.4.3) and combine it with the statistical errors by root sum square. We rejected a small number of sources detected at $\mathrm{S} / \mathrm{N}<5$, where the systematic errors may be larger. The measured fluxes and resulting errors for the remaining sample of 139 quasars are provided in Table 2.

To extend the time sampling to 2010, we collected WISE W4 measurements at $22 \mu \mathrm{m}$ for 136 of these objects from the ALLWISE Source Catalog. One WISE measurement was rejected because of confusion with a nearby source. The W4 and MIPS [24] photometric bands are similar, with a cut-on wavelength at $19.87 \mu \mathrm{m}$ (half power) for the former and $20.80 \mu \mathrm{m}$ for the latter, with the long wavelength response determined by the Si:As IBC detectors in both cases.
Low-resolution $(\lambda / \Delta \lambda \sim 60-130)$ mid-IR spectroscopic observations of all 87 PG quasars were obtained using the Spitzer IRS. By comparing them with the WISE W3 and W4 photometric measurements, we can study quasar variability at 10-22 $\mu \mathrm{m}$. For the Spitzer mid-IR specta, we adopted the optimal extraction products from the Combined Atlas of Sources with Spitzer IRS Spectra (CASSIS; Lebouteiller et al. 2011). Besides PG 0003+199 (no Long-Low module observation at 14-38 $\mu \mathrm{m}$ ) and PG 1352 +183 (very poor Short-Low spectra at 5.2-14 $\mu \mathrm{m}$ ), the mid-IR spectra cover $\sim 5.2-24 \mu \mathrm{m}$ for all objects and with good S/Ns. Compared with the WISE measurements in 2010, these Spitzer/ IRS spectra were typically obtained 5 yr earlier. For PG $1226+023$ (3C 273), multiple Spitzer/IRS observations were conducted between 2004 and 2009, making possible the construction of midIR light curves over a wide wavelength range. For the WISE W3 and W4 measurements of these PG quasars, since no convincing variability has been reported in these bands over the 9-month cryogenic mission by the WISE pipeline, we adopted the profile-fit photometry values from the ALLWISE source catalog.

\subsection{Construction and Evaluation of the Light Curves}

In the optical and mid-IR $W 1$ and $W 2$ bands, we have sufficient data to sample light curves for many years. In this section, we discuss the procedures developed for this purpose.

\subsubsection{Optical}

Typically, the CRTS data cover MJD 53,500-56,500, and the ASAS-SN data cover MJD 56,000-58,300, with an 

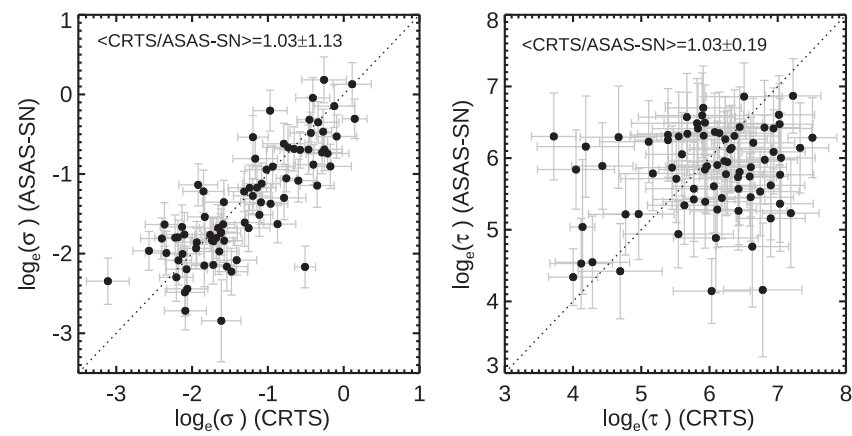

Figure 2. Comparisons of the best-fit DRW model parameters from fitting the ASAS-SN and CRTS light curves separately with the JAVELIN code. The left panel shows the amplitude $\sigma$ of the fitted covariance function, and the right panel is the corresponding damping timescale $\tau$. We denote the average parameter ratio with the corresponding standard deviation between CRTS and ASAS-SN light curves at the top of each panel.

overlap of about 500 days. We rely on these data sets to construct the optical light curves for most objects. The CRTS data generally have smaller relative uncertainties. However, the absolute values of the unfiltered photometry are hard to interpret, so we scaled the CRTS data to match the ASAS-SN data in the region of overlap; the latter have photometry measurements obtained through a standard $V$-band filter and calibrated by nearby standard stars. Since CRTS and ASAS-SN observations have different time samplings, we interpolated the observed values with the same time grid by fitting a damped random walk (DRW) model to the CRTS and ASAS-SN light curves separately and computed an average scaling factor for the best-fit model light curves with the shared time periods.

To demonstrate the validity of using the combined unfiltered CRTS and $V$-band ASAS-SN light curves to trace the accretion disk variability, we compare the derived best-fit DRW parameters for these two data sets in Figure 2. We have used JAVELIN (Zu et al. 2013) to fit the slightly smoothed optical light curves $^{5}$ and the derived best-fit parameters using an MCMC analysis. They are in excellent agreement despite the large uncertainties of individual values, suggesting the CRTS and ASAS-SN light curves trace identical variability properties. In terms of the wavelength dependence of the AGN optical continuum variability, Jiang et al. (2017) found that the average time delays between $g(\sim 0.48 \mu \mathrm{m})$ and $z(\sim 0.91 \mu \mathrm{m})$ are less than 6 days for quasars with $L_{\mathrm{AGN}, \text { bol }} \sim 10^{11}-10^{13} L_{\odot}$. This is at least 1 order of magnitude smaller than the dust reverberation lags our data can probe. Consequently, we do not need to make any corrections for the filter differences between the CRTS and ASAS-SN data sets. In fact, we have also visually checked the CRTS and ASAS-SN combined light curves of individual objects, finding that they have consistent features, as revealed by the PTF light curves. Figure 3 gives some examples of how this model works on real data.

Among 87 PG quasars, four objects (PG 0804+761, PG 0838+761, PG 1100+772, and PG 1427+480) do not have CRTS observations. In these cases, we join the PTF and ASASSN data together to extend the time coverage of their optical light curves. The objects PG 1354+213 and PG 1416-129 are too faint for the ASAS-SN photometry to be useful. We have also dropped the CRTS light curve of PG 1226+023, since the photometry measurements are saturated.

\footnotetext{
5 A 1 day smoothing window is introduced for both CRTS and ASAS-SN light curves to reduce the photometry uncertainties before the fittings.
}

For some objects, there are spike features in the optical light curves, which commonly turned out to be one single photometry measurement larger (or smaller) than the nearby average values by 1-2 mag. This is quite likely unrelated to the AGN itself but caused by, e.g., poor seeing conditions, calibration errors, or contamination, such as cosmic rays. We rejected such data points by introducing $3 \sigma$ clipping and smoothed the final optical light curve by averaging the remaining measurements taken within a single day to reduce the noise.

In the bottom right panel of Figure 3, we compare the optical $V$-band light curves of PG $1226+023$ from different sources. Although AAVSO and ASAS-SN measurements have larger dispersions than the data obtained from dedicated monitoring with the SO telescopes, these light curves reveal the same variability patterns for this object. We decide to adopt the SO light curve for the correlation analysis between the optical and mid-IR variability and combine it with the AAVSO data to increase the time-sampling range if required.

\subsubsection{WISE $\mathrm{W} 1$ and $\mathrm{W} 2$}

A single WISE epoch normally contains 10-20 7.7 s individual exposures with a total time coverage of about 1 day. For the midIR variability traced by the WISE data, we have first visually investigated the consistency of WISE W1 and W2 single-exposure photometry: they should have similar variability features given their small wavelength difference. This is confirmed for the available epochs of most objects. For PG 1259+593, PG 1534 +580 , and PG $1700+518$, there are only one to two photometry spikes in the single-epoch light curves, and they can be easily rejected. There are uncorrelated $W 1$ and $W 2$ variability signals in one-third of the epochs for PG 0923+129. Finally, PG 1617 +175 has many abnormal Wl-band photometry values in the two epochs observed in the cryogenic survey, whose variability features are not found in the $W 2$ band. We have rejected these problematic measurements.

We are interested in the response signal of the dust emission; the signal in the WISE bands will therefore arise near the inner radius of the torus around the central engine, and any variations should be much slower than 1 light-day. Therefore, we averaged the photometry values during each 1 day epoch to compile the mid-IR light curve, i.e.,

$$
f_{\text {epoch }}=\frac{1}{N} \sum_{i=1}^{N} f_{i}
$$

Standard deviations of the single-exposure photometry were also derived to estimate the measurement uncertainties for each epoch. In addition, uncertainties in photometric measurements $\left(\sigma_{i, \text { pho }}\right)$, as well as the system stability $\left(\sigma_{\text {s.s. }}\right)$, need to be considered, so the total flux uncertainty for a given epoch is

$$
\sigma_{\text {epoch }}^{2}=\frac{1}{N-1} \sum_{i=1}^{N}\left(f_{i}-\overline{f_{\text {epoch }}}\right)^{2}+\frac{1}{N^{2}} \sum_{i=1}^{N} \sigma_{i, \text { pho }}^{2}+\frac{1}{N} \sigma_{\text {s.s. }}^{2} .
$$

In Appendix A, we study the photometric stability of WISE and NEOWISE using mid-IR calibration stars, finding $\sigma_{\text {s.s. }} \sim 0.029$ mag for WISE measurements and $\sigma_{\text {s.s. }} \sim 0.016 \mathrm{mag}$ for NEOWISE measurements.

For both optical and mid-IR light curves, we converted magnitudes into flux to better reflect the variability signals from the AGN, since various contaminations (e.g., AGN host galaxy, 

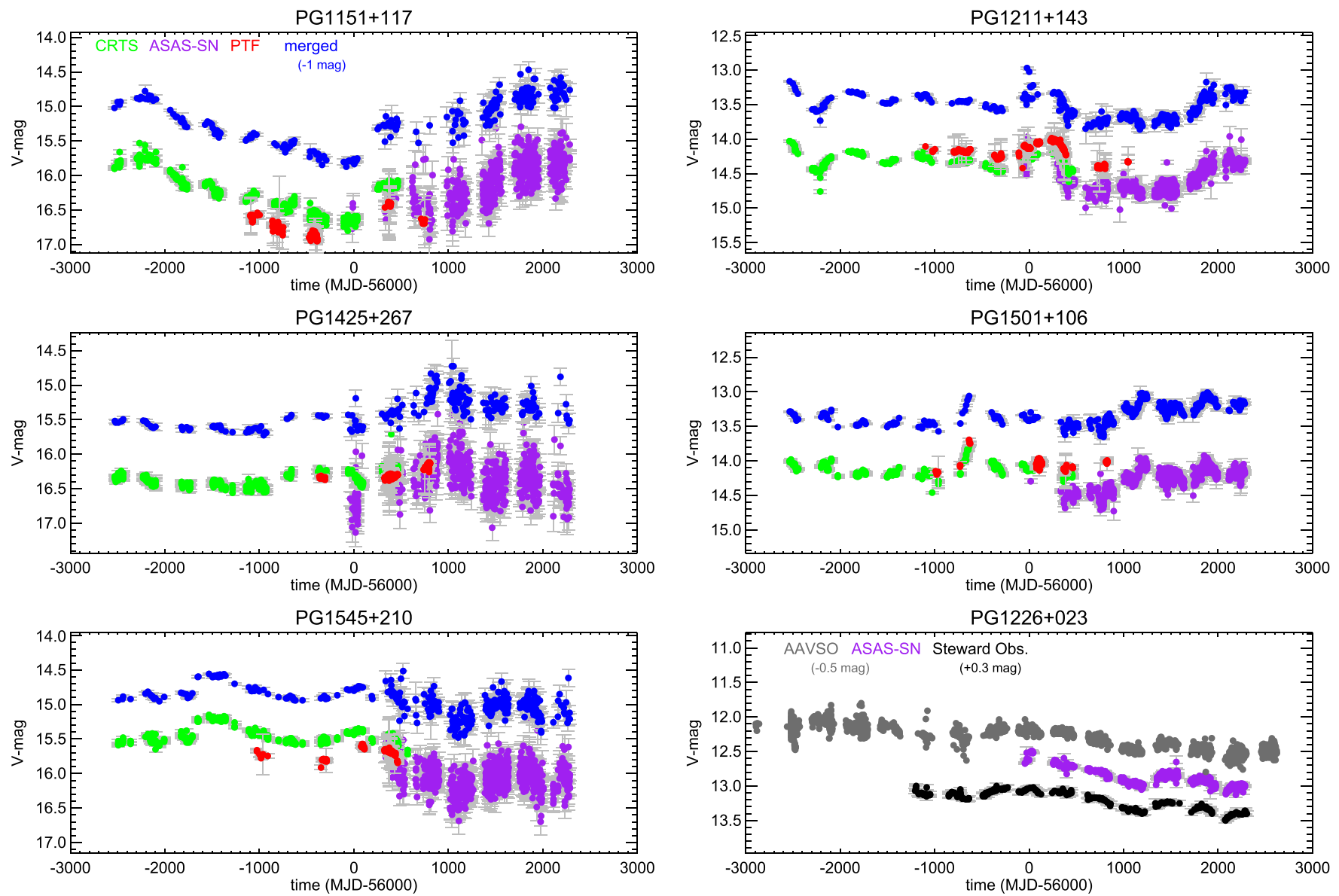

Figure 3. Example optical light curves of PG quasars. The magnitude measurements from ASAS-SN (purple), CRTS (green), and PTF (red) are shown with the original values in their catalog without any shifts. The final combined light curves (blue data points) are shifted by 1 mag for clarity. For PG $1226+023$ (bottom right panel), we compare its light curves from AAVSO (gray; shifted by $-0.5 \mathrm{mag}$ ), ASAS-SN (purple; no shift), and observations obtained with two SO telescopes (black; shifted by $+0.3 \mathrm{mag})$.

nearby objects) and photometry zero-point uncertainties would only cause a constant flux shift.

In Table 3, we provide a summary of available light-curve data and their basic properties for the PG sample. Interested readers may contact the authors directly to get a copy of these light curves.

\subsection{Converting the Mid-IR Measurements at $\lambda>10 \mu \mathrm{m}$ for Comparison}

We do not have sufficient data to construct light curves in the $10-25 \mu \mathrm{m}$ range, but we can assess variability by comparing the individual measurements. We describe here how we extended this comparison beyond just the MIPS $24 \mu \mathrm{m}$ data.

\subsubsection{WISE W4 to MIPS [24]}

To convert the WISE W4 measurements to the MIPS [24] system, we first eliminated all quasars in our sample that we found to vary, plus those with $W 4$ measurement errors $\geqslant 0.08$ mag. These cuts left 95 quasars with high-weight measurements in both systems. We took the zero-point for [24] to be $7.17 \mathrm{Jy}$ and based the [24] magnitude on the weighted average of the two measurements of each quasar. A linear fit, which is specific to quasar SEDs, results in a transformation equation of

$$
\begin{aligned}
W 4-[24]= & 0.014 \pm 0.036+(0.024 \pm 0.015) \\
& \times(W 3-W 4) .
\end{aligned}
$$

We applied Equation (3) to the WISE data and assigned the date corresponding to the averaged Modified Julian Date (MJD) of the WISE single exposures from the WISE All-Sky Data Release Source Catalog. To allow for uncertainties in the transformation, as well as potential systematic uncertainties in the WISE measurements, we augmented the quoted errors by 0.02 mag (see Appendix A) as the root sum square with the nominal error. We then calculated the change between the weighted average of the first Spitzer measurements and the WISE one. Table 2 shows the transformed WISE measurement, its estimated uncertainty, and the number of standard deviations of difference between it and the Spitzer measurements. Sources with changes $>3 \sigma$ between the Spitzer and WISE measurements and among the Spitzer measurements themselves are indicated as variable in the comments column.

The slope coefficient in Equation (3) is consistent with zero, so we have tested the reliability of the results by simply averaging the ratios of the two measurements to derive a colorindependent transformation. Using these results slightly increased the apparent significance level of the changes, typically by about $0.1 \sigma$. In general, there would be no change in the designation of variable sources with this alternative transformation, with the exception of Cygnus $\mathrm{A}$, which is changed from being marginally not variable $(2.5 \sigma)$ to marginally variable $(3.4 \sigma)$. That is, the results are relatively robust against modest errors in the transformation. This is not 
Table 3

Summary of Available Data and Basic Variability Properties

\begin{tabular}{|c|c|c|c|c|c|c|c|c|c|c|c|c|c|c|}
\hline \multirow{2}{*}{$\begin{array}{l}\text { Name } \\
\text { (1) }\end{array}$} & \multicolumn{3}{|c|}{ Optical Sources } & \multirow{2}{*}{$\begin{array}{c}\delta M_{V} \\
(5)\end{array}$} & \multirow{2}{*}{$\begin{array}{c}\operatorname{MJD}(V) \\
(6)\end{array}$} & \multirow{2}{*}{$\begin{array}{c}N_{\text {epoch }} \\
\text { (7) }\end{array}$} & \multirow{2}{*}{$\begin{array}{l}V \\
(8)\end{array}$} & \multirow{2}{*}{$\begin{array}{l}\Delta V \\
(9)\end{array}$} & \multirow{2}{*}{$\begin{array}{c}\text { MJD(IR) } \\
(10)\end{array}$} & \multirow{2}{*}{$\begin{array}{c}N_{\text {epoch }} \\
(11)\end{array}$} & \multirow{2}{*}{$\begin{array}{l}W 1 \\
\text { (12) }\end{array}$} & \multirow{2}{*}{$\begin{array}{l}\Delta W 1 \\
(13)\end{array}$} & \multirow{2}{*}{$\begin{array}{l}W 2 \\
(14)\end{array}$} & \multirow{2}{*}{$\begin{array}{r}\Delta W 2 \\
(15)\end{array}$} \\
\hline & $\begin{array}{l}\text { CRTS } \\
\text { (2) }\end{array}$ & $\begin{array}{c}\text { ASAS-SN } \\
\text { (3) }\end{array}$ & $\begin{array}{l}\text { PTF } \\
(4)\end{array}$ & & & & & & & & & & & \\
\hline PG $0003+158$ & I & I & II & 0.19 & $53,648.3-58,281.1$ & 325 & 15.63 & 1.03 & $55,376.1-58,459.7$ & 13 & 12.27 & 0.06 & 11.22 & 0.11 \\
\hline PG 0003+199 & I & I & II & 0.33 & $53,648.3-58,281.1$ & 382 & 14.16 & 0.46 & $55,377.8-58,461.4$ & 14 & 8.75 & 0.54 & 7.84 & 0.43 \\
\hline PG $0007+106$ & I & I & III & 0.42 & $53,520.5-58,282.1$ & 344 & 15.43 & 0.85 & $55,375.0-58,458.8$ & 13 & 10.55 & 0.38 & 9.58 & 0.27 \\
\hline PG $0026+129$ & I & I & III & 0.32 & $53,520.5-58,282.1$ & 381 & 15.33 & 1.82 & $55,379.5-58,463.5$ & 13 & 11.10 & 0.24 & 10.10 & 0.19 \\
\hline PG $0043+039$ & I & I & III & 0.14 & $53,563.4-58,284.1$ & 260 & 15.91 & 0.78 & $55,559.1-58,463.5$ & 11 & 12.02 & 0.16 & 10.95 & 0.12 \\
\hline PG $0049+171$ & I & I & N.A. & 0.28 & $53,636.4-58,281.1$ & 369 & 15.57 & 1.20 & $55,203.5-58,311.3$ & 13 & 11.79 & 0.24 & 10.84 & 0.19 \\
\hline PG $0050+124$ & I & I & III & 0.41 & $53,636.4-58,273.1$ & 380 & 14.12 & 0.54 & $55,387.5-58,309.8$ & 12 & 8.77 & 0.30 & 7.77 & 0.21 \\
\hline PG $0052+251$ & I & I & III & 0.26 & $53,561.4-58,138.7$ & 378 & 15.40 & 1.00 & $55,206.4-58,314.9$ & 16 & 11.08 & 0.20 & 10.09 & 0.18 \\
\hline PG $0157+001$ & I & I & II & 0.22 & $53,627.4-58,158.5$ & 315 & 15.46 & 0.82 & $55,210.8-58,319.8$ & 13 & 10.80 & 0.43 & 9.81 & 0.31 \\
\hline PG 0804+761 & N.A. & I & I & 0.22 & $55,312.3-58,271.7$ & 388 & 14.25 & 0.75 & $55,284.5-58,398.4$ & 14 & 9.37 & 0.33 & 8.34 & 0.22 \\
\hline PG $0838+770$ & N.A. & I & I & 0.22 & $55,312.3-58,257.8$ & 180 & 15.95 & 1.00 & $55,286.8-58,400.7$ & 15 & 11.58 & 0.31 & 10.66 & 0.27 \\
\hline PG $0844+349$ & I & I & II & 0.24 & $53,495.2-58,254.8$ & 385 & 14.36 & 0.82 & $55,305.2-58,418.5$ & 11 & 10.49 & 0.29 & 9.63 & 0.22 \\
\hline PG $0921+525$ & I & I & II & 0.27 & $53,714.4-58,260.8$ & 383 & 14.36 & 0.93 & $55,305.9-58,422.9$ & 18 & 10.24 & 0.55 & 9.30 & 0.41 \\
\hline PG 0923+201 & I & I & III & 0.29 & $53,469.2-58,228.6$ & 352 & 15.24 & 0.95 & $55,319.5-58,432.8$ & 12 & 10.48 & 0.16 & 9.55 & 0.11 \\
\hline PG $0923+129$ & I & I & II & 0.45 & $53,500.2-58,264.5$ & 421 & 14.26 & 0.56 & $55,321.7-58,434.8$ & 12 & 10.16 & 0.36 & 9.27 & 0.31 \\
\hline PG 0934+013 & I & I & III & 0.30 & $53,464.2-58,223.7$ & 347 & 15.56 & 0.76 & $55,327.8-58,440.6$ & 13 & 11.34 & 0.82 & 10.41 & 0.77 \\
\hline PG 0947+396 & I & I & III & 0.24 & $53,537.2-58,252.8$ & 168 & 16.41 & 1.02 & $55,318.0-58,431.2$ & 15 & 11.75 & 0.44 & 10.59 & 0.43 \\
\hline PG $0953+414$ & I & I & II & 0.09 & $53,711.4-58,261.4$ & 337 & 14.88 & 0.84 & $55,318.3-58,431.6$ & 14 & 10.74 & 0.27 & 9.76 & 0.17 \\
\hline PG $1001+054$ & I & I & II & -0.08 & $53,464.2-58,246.6$ & 298 & 16.17 & 1.82 & $55,332.6-58,445.1$ & 12 & 11.42 & 0.24 & 10.41 & 0.25 \\
\hline PG $1004+130$ & I & I & II & 0.19 & $53,527.2-58,264.5$ & 256 & 15.33 & 0.60 & $55,330.7-58,443.3$ & 13 & 11.58 & 0.18 & 10.48 & 0.13 \\
\hline PG 1011-040 & I & I & III & 0.31 & $53,496.1-58,230.6$ & 364 & 14.93 & 0.78 & $55,340.5-58,453.2$ & 13 & 11.08 & 0.25 & 10.21 & 0.24 \\
\hline PG $1012+008$ & I & I & III & 0.27 & $53,464.2-58,228.6$ & 335 & 15.62 & 1.02 & $55,339.3-58,448.9$ & 12 & 11.36 & 0.35 & 10.37 & 0.23 \\
\hline PG $1022+519$ & I & I & II & 0.21 & $53,714.4-58,271.8$ & 263 & 15.41 & 0.81 & $55,318.4-58,431.8$ & 15 & 11.35 & 0.42 & 10.53 & 0.42 \\
\hline PG $1048+342$ & I & I & III & 0.22 & $53,496.0-58,275.8$ & 227 & 16.52 & 0.71 & $55,332.0-58,444.6$ & 15 & 12.36 & 0.24 & 11.34 & 0.27 \\
\hline PG 1048-090 & I & I & III & -0.51 & $53,707.7-58,233.5$ & 345 & 15.44 & 0.62 & $55,350.8-58,462.9$ & 14 & 11.98 & 0.06 & 10.97 & 0.06 \\
\hline PG 1049-005 & I & I & N.A. & 0.23 & $53,464.3-58,230.7$ & 303 & 15.99 & 0.99 & $55,347.7-58,459.8$ & 12 & 11.22 & 0.10 & 10.10 & 0.07 \\
\hline PG $1100+772$ & N.A. & I & I & 0.23 & $55,294.4-58,281.8$ & 338 & 15.26 & 0.64 & $55,297.3-58,410.9$ & 14 & 11.29 & 0.17 & 10.23 & 0.17 \\
\hline PG 1103-006 & I & I & III & -0.07 & $53,464.3-58,231.9$ & 310 & 16.05 & 1.11 & $55,351.0-58,462.9$ & 13 & 12.21 & 0.08 & 11.09 & 0.10 \\
\hline PG $1114+445$ & I & I & II & 0.18 & $53,531.2-58,280.8$ & 261 & 15.83 & 0.86 & $55,331.7-58,451.9$ & 18 & 10.88 & 0.10 & 9.73 & 0.11 \\
\hline PG $1115+407$ & I & I & N.A. & 0.16 & $53,527.2-58,280.8$ & 307 & 15.76 & 0.65 & $55,334.1-58,446.5$ & 15 & 11.33 & 0.22 & 10.42 & 0.13 \\
\hline PG $1116+215$ & I & I & III & 0.17 & $53,469.3-58,283.8$ & 373 & 14.71 & 1.83 & $55,345.2-58,457.6$ & 12 & 10.02 & 0.26 & 9.01 & 0.23 \\
\hline PG $1119+120$ & I & I & III & 0.26 & $53,498.2-58,283.8$ & 368 & 14.62 & 0.96 & $55,349.6-58,461.6$ & 12 & 10.55 & 0.27 & 9.55 & 0.14 \\
\hline PG $1121+422$ & I & I & N.A. & 0.14 & $53,527.2-58,280.8$ & 261 & 16.25 & 1.17 & $55,334.3-58,452.1$ & 17 & 12.13 & 0.38 & 11.15 & 0.38 \\
\hline PG 1126-041 & I & I & II & 0.19 & $53,496.2-58,230.5$ & 375 & 14.48 & 0.63 & $55,357.4-58,263.6$ & 13 & 9.70 & 0.30 & 8.78 & 0.27 \\
\hline PG 1149-110 & I & I & III & 0.54 & $53,479.2-58,275.8$ & 360 & 15.11 & 3.18 & $55,364.7-58,271.2$ & 12 & 11.06 & 0.55 & 10.34 & 0.57 \\
\hline PG $1151+117$ & I & I & II & 0.13 & $53,466.2-58,283.8$ & 230 & 16.15 & 1.40 & $55,356.8-58,259.8$ & 12 & 11.87 & 0.51 & 10.87 & 0.30 \\
\hline PG $1202+281$ & I & I & II & 0.14 & $53,470.3-58,254.9$ & 238 & 16.35 & 1.16 & $55,352.1-58,463.8$ & 14 & 11.42 & 0.44 & 10.48 & 0.32 \\
\hline PG $1211+143$ & I & I & II & 0.13 & $53,466.3-58,284.0$ & 364 & 14.55 & 0.89 & $55,360.1-58,266.3$ & 11 & 10.06 & 0.26 & 8.96 & 0.27 \\
\hline PG $1216+069$ & I & I & I & 0.13 & $55,270.4-58,282.8$ & 272 & 13.42 & 0.57 & $55,364.1-58,270.3$ & 12 & 11.63 & 0.14 & 10.68 & 0.10 \\
\hline PG $1226+023$ & III & I & N.A. & 0.00 & $55,956.1-58,283.8$ & 299 & 12.87 & 0.61 & $55,370.4-58,274.3$ & 12 & 8.41 & 0.16 & 7.45 & 0.11 \\
\hline PG $1229+204$ & I & I & II & 0.29 & $53,469.3-58,282.8$ & 424 & 14.89 & 0.66 & $55,361.5-58,267.6$ & 15 & 10.64 & 0.48 & 9.73 & 0.42 \\
\hline PG $1244+026$ & I & I & II & 0.31 & $53,767.3-58,283.8$ & 276 & 15.82 & 0.74 & $55,374.2-58,277.9$ & 12 & 11.63 & 0.08 & 10.58 & 0.06 \\
\hline PG $1259+593$ & I & I & II & 0.06 & $53,767.3-58,279.8$ & 309 & 15.44 & 0.62 & $55,336.9-58,452.8$ & 16 & 11.25 & 0.18 & 10.22 & 0.13 \\
\hline PG 1302-102 & I & I & N.A. & 0.16 & $53,496.2-58,274.7$ & 347 & 15.21 & 0.68 & $55,208.7-58,286.7$ & 13 & 11.38 & 0.24 & 10.28 & 0.17 \\
\hline PG $1307+085$ & I & I & N.A. & 0.14 & $53,466.3-58,283.8$ & 315 & 15.62 & 0.74 & $55,377.2-58,280.7$ & 12 & 11.41 & 0.28 & 10.36 & 0.23 \\
\hline PG $1309+355$ & I & I & II & 0.15 & $53,526.2-58,284.9$ & 360 & 15.36 & 0.64 & $55,362.7-58,268.9$ & 17 & 11.14 & 0.13 & 10.07 & 0.08 \\
\hline
\end{tabular}


Table 3

(Continued)

\begin{tabular}{|c|c|c|c|c|c|c|c|c|c|c|c|c|c|c|}
\hline \multirow{2}{*}{$\begin{array}{l}\text { Name } \\
\text { (1) }\end{array}$} & \multicolumn{3}{|c|}{ Optical Sources } & \multirow{2}{*}{$\begin{array}{c}\delta M_{V} \\
(5)\end{array}$} & \multirow{2}{*}{$\begin{array}{c}\operatorname{MJD}(V) \\
(6)\end{array}$} & \multirow{2}{*}{$\begin{array}{c}N_{\text {epoch }} \\
\text { (7) }\end{array}$} & \multirow{2}{*}{$\begin{array}{l}V \\
(8)\end{array}$} & \multirow{2}{*}{$\begin{array}{l}\Delta V \\
(9)\end{array}$} & \multirow{2}{*}{$\begin{array}{c}\text { MJD(IR) } \\
(10)\end{array}$} & \multirow{2}{*}{$\begin{array}{c}N_{\text {epoch }} \\
(11)\end{array}$} & \multirow{2}{*}{$\begin{array}{l}W 1 \\
(12)\end{array}$} & \multirow{2}{*}{$\begin{array}{l}\Delta W 1 \\
(13)\end{array}$} & \multirow{2}{*}{$\begin{array}{l}W 2 \\
(14)\end{array}$} & \multirow{2}{*}{$\begin{array}{c}\Delta W 2 \\
(15)\end{array}$} \\
\hline & $\begin{array}{l}\text { CRTS } \\
\text { (2) }\end{array}$ & $\begin{array}{l}\text { ASAS-SN } \\
\text { (3) }\end{array}$ & $\begin{array}{l}\mathrm{PTF} \\
(4)\end{array}$ & & & & & & & & & & & \\
\hline PG 1310-108 & I & I & N.A. & 0.26 & $53,496.2-58,274.7$ & 349 & 15.24 & 0.62 & $55,210.2-58,288.5$ & 13 & 11.21 & 0.16 & 10.08 & 0.20 \\
\hline PG $1322+659$ & I & I & II & -0.04 & $53,860.4-58,279.8$ & 281 & 15.56 & 0.87 & $55,330.8-58,451.9$ & 18 & 11.48 & 0.33 & 10.48 & 0.23 \\
\hline PG $1341+258$ & I & I & II & 0.06 & $53,470.3-58,283.9$ & 375 & 15.63 & 1.16 & $55,204.0-58,281.2$ & 16 & 11.49 & 0.37 & 10.66 & 0.30 \\
\hline PG $1351+236$ & I & I & III & 0.27 & $53,470.3-58,284.9$ & 356 & 15.27 & 0.80 & $55,207.3-58,291.2$ & 17 & 11.37 & 0.31 & 10.74 & 0.34 \\
\hline PG $1351+640$ & I & I & II & 0.00 & $53,767.5-58,284.9$ & 390 & 14.77 & 0.76 & $55,337.1-58,453.2$ & 17 & 10.28 & 0.08 & 9.19 & 0.11 \\
\hline PG $1352+183$ & I & I & II & 0.18 & $53,469.4-58,284.9$ & 272 & 16.13 & 0.80 & $55,208.9-58,291.4$ & 16 & 11.71 & 0.31 & 10.68 & 0.28 \\
\hline PG $1354+213$ & I & III & II & 0.00 & $53,469.4-56,447.2$ & 80 & 16.64 & 0.78 & $55,208.4-58,291.3$ & 17 & 12.69 & 0.50 & 11.56 & 0.48 \\
\hline PG $1402+261$ & I & I & II & 0.08 & $53,470.3-58,283.9$ & 363 & 15.61 & 0.65 & $55,208.4-58,285.9$ & 16 & 10.60 & 0.25 & 9.54 & 0.22 \\
\hline PG $1404+226$ & I & I & N.A. & 0.13 & $53,469.4-58,284.9$ & 298 & 15.80 & 0.77 & $55,209.7-58,287.8$ & 15 & 11.81 & 0.24 & 10.89 & 0.19 \\
\hline PG $1411+442$ & I & I & II & 0.22 & $53,509.4-58,284.8$ & 355 & 14.73 & 0.67 & $55,372.8-58,276.5$ & 15 & 10.11 & 0.16 & 9.07 & 0.16 \\
\hline PG $1415+451$ & I & I & II & 0.17 & $53,509.4-58,284.8$ & 317 & 15.79 & 0.74 & $55,372.7-58,276.5$ & 15 & 11.33 & 0.44 & 10.44 & 0.33 \\
\hline PG 1416-129 & I & III & N.A. & 0.00 & $53,498.3-56,478.5$ & 100 & 16.92 & 0.74 & $55,222.9-58,306.4$ & 13 & 12.30 & 0.43 & 11.32 & 0.37 \\
\hline PG $1425+267$ & I & $\mathrm{I}$ & II & 0.18 & $53,470.3-58,281.9$ & 224 & 16.36 & 1.00 & $55,212.5-58,293.7$ & 17 & 11.98 & 0.09 & 10.88 & 0.07 \\
\hline PG $1426+015$ & I & I & II & 0.25 & $53,464.4-58,283.9$ & 378 & 14.32 & 0.66 & $55,221.0-58,304.2$ & 13 & 9.95 & 0.26 & 9.06 & 0.17 \\
\hline PG $1427+480$ & N.A. & I & I & 0.25 & $54,962.3-58,284.8$ & 402 & 16.39 & 2.28 & $55,373.0-58,276.8$ & 15 & 12.25 & 0.15 & 11.18 & 0.11 \\
\hline PG 1435-067 & I & I & N.A. & 0.22 & $53,497.3-58,283.9$ & 330 & 15.80 & 0.84 & $55,225.1-58,308.8$ & 13 & 11.40 & 0.29 & 10.34 & 0.20 \\
\hline PG $1440+356$ & I & I & II & 0.17 & $53,480.3-58,281.9$ & 390 & 14.61 & 0.72 & $55,211.4-58,292.7$ & 17 & 9.93 & 0.23 & 9.07 & 0.23 \\
\hline PG $1444+407$ & I & I & III & 0.16 & $53,509.4-58,281.9$ & 313 & 15.71 & 0.75 & $55,209.3-58,291.4$ & 18 & 11.36 & 0.15 & 10.33 & 0.14 \\
\hline PG $1448+273$ & I & I & II & 0.15 & $53,470.4-58,283.9$ & 382 & 14.72 & 0.64 & $55,217.2-58,299.9$ & 18 & 10.94 & 0.41 & 10.05 & 0.33 \\
\hline PG $1501+106$ & I & I & II & 0.28 & $53,466.4-58,282.9$ & 403 & 14.29 & 0.64 & $55,225.8-58,309.5$ & 14 & 10.19 & 0.30 & 9.20 & 0.21 \\
\hline PG $1512+370$ & I & I & II & 0.23 & $53,480.3-58,281.9$ & 277 & 16.19 & 1.04 & $55,217.9-58,300.5$ & 18 & 12.19 & 0.09 & 11.07 & 0.10 \\
\hline PG $1519+226$ & I & I & II & 0.20 & $53,506.3-58,284.9$ & 355 & 15.86 & 1.08 & $55,225.9-58,309.6$ & 16 & 10.82 & 0.34 & 9.88 & 0.21 \\
\hline PG $1534+580$ & I & I & II & 0.31 & $53,856.4-58,281.8$ & 344 & 14.55 & 0.44 & $55,203.3-58,278.5$ & 16 & 10.41 & 0.51 & 9.44 & 0.47 \\
\hline PG $1535+547$ & I & I & II & 0.17 & $53,880.5-58,284.8$ & 404 & 14.67 & 0.71 & $55,203.1-58,291.3$ & 18 & 9.91 & 0.55 & 9.07 & 0.42 \\
\hline PG $1543+489$ & I & I & II & 0.06 & $53,531.3-58,277.9$ & 202 & 16.24 & 0.82 & $55,216.8-58,299.2$ & 19 & 11.52 & 0.13 & 10.40 & 0.13 \\
\hline PG $1545+210$ & I & I & II & 0.37 & $53,506.4-58,284.9$ & 324 & 15.98 & 0.96 & $55,232.7-58,320.9$ & 17 & 11.62 & 0.24 & 10.56 & 0.23 \\
\hline PG $1552+085$ & I & I & III & 0.12 & $53,466.4-58,284.9$ & 379 & 15.59 & 0.81 & $55,241.0-58,325.8$ & 15 & 11.58 & 0.37 & 10.66 & 0.31 \\
\hline PG $1612+261$ & I & I & II & 0.06 & $53,470.4-58,281.9$ & 413 & 15.42 & 0.99 & $55,241.2-58,325.9$ & 16 & 11.01 & 0.15 & 10.10 & 0.15 \\
\hline PG $1613+658$ & I & I & II & 0.15 & $53,856.4-58,281.9$ & 330 & 14.65 & 0.57 & $55,355.0-58,464.9$ & 18 & 10.16 & 0.14 & 9.15 & 0.09 \\
\hline PG $1617+175$ & I & I & II & 0.12 & $53,469.4-58,283.8$ & 420 & 15.16 & 0.88 & $55,245.4-58,330.4$ & 16 & 10.40 & 0.30 & 9.48 & 0.27 \\
\hline PG $1626+554$ & I & I & II & 0.19 & $53,505.4-58,281.8$ & 341 & 15.65 & 1.03 & $55,221.1-58,304.2$ & 17 & 11.39 & 0.24 & 10.47 & 0.18 \\
\hline PG $1700+518$ & I & I & II & 0.18 & $53,505.4-58,281.2$ & 374 & 14.89 & 0.49 & $55,242.8-58,327.9$ & 17 & 10.26 & 0.04 & 9.20 & 0.04 \\
\hline PG $1704+608$ & I & I & II & 0.18 & $53,856.4-58,284.9$ & 347 & 15.34 & 0.91 & $55,223.3-58,306.5$ & 16 & 10.79 & 0.06 & 9.73 & 0.06 \\
\hline PG $2112+059$ & I & I & III & 0.19 & $53,466.5-58,284.0$ & 415 & 15.42 & 0.77 & $55,330.9-58,416.8$ & 13 & 10.99 & 0.12 & 9.83 & 0.07 \\
\hline PG $2130+099$ & I & I & II & 0.31 & $53,466.5-58,284.0$ & 419 & 14.54 & 0.76 & $55,336.4-58,422.1$ & 14 & 9.56 & 0.34 & 8.59 & 0.25 \\
\hline PG $2209+184$ & I & I & II & 0.41 & $53,480.5-58,284.0$ & 385 & 15.54 & 0.87 & $55,348.9-58,439.7$ & 15 & 11.40 & 0.50 & 10.61 & 0.62 \\
\hline PG 2214+139 & I & I & II & 0.31 & $53,554.4-58,282.1$ & 396 & 14.42 & 0.49 & $55,348.5-58,439.6$ & 13 & 9.77 & 0.12 & 8.88 & 0.07 \\
\hline PG $2233+134$ & I & I & II & 0.19 & $53,531.4-58,284.0$ & 276 & 16.34 & 0.96 & $55,355.1-58,440.4$ & 15 & 12.04 & 0.19 & 10.88 & 0.16 \\
\hline PG $2251+113$ & I & I & II & 0.33 & $53,531.4-58,282.0$ & 340 & 15.67 & 0.63 & $55,358.4-58,443.3$ & 12 & 10.98 & 0.03 & 10.07 & 0.04 \\
\hline PG 2304+042 & I & I & II & 0.51 & $53,553.4-58,281.6$ & 362 & 15.14 & 2.28 & $55,358.5-58,443.5$ & 12 & 11.04 & 1.01 & 10.42 & 1.05 \\
\hline PG $2308+098$ & I & I & II & 0.17 & $53,506.5-58,282.0$ & 283 & 16.06 & 1.11 & $55,361.6-58,446.5$ & 11 & 12.02 & 0.24 & 10.93 & 0.23 \\
\hline
\end{tabular}

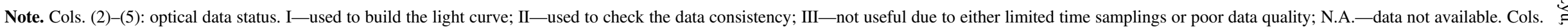

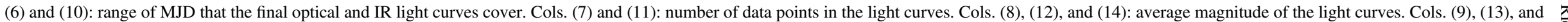
(15): variation amplitude of the light curves. 


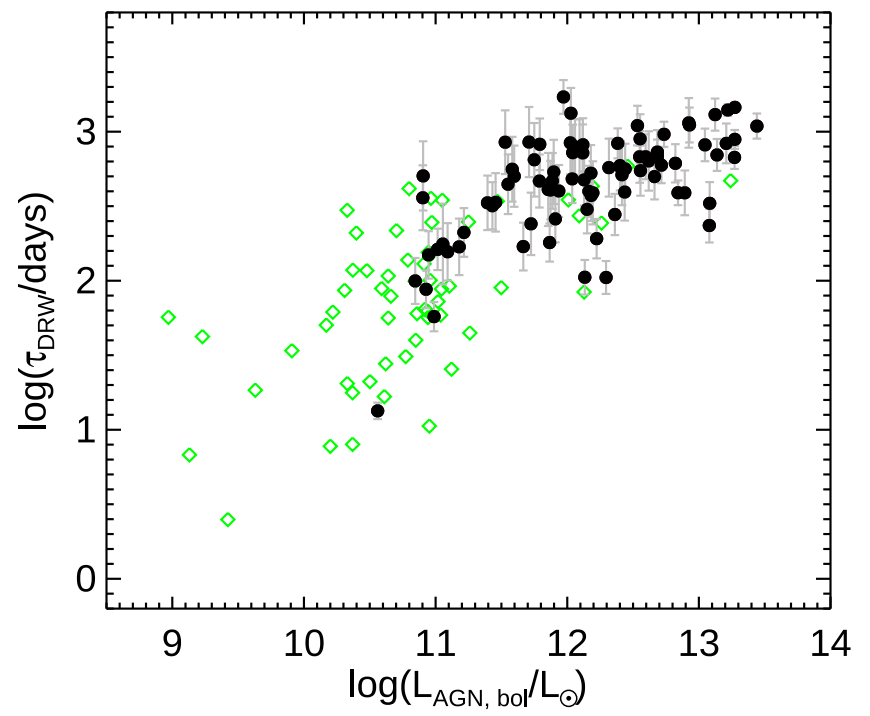

Figure 4. Rest-frame DRW damping timescale, $\tau_{\mathrm{DRM}}$, of the optical light curves as a function of AGN bolometric luminosity, $L_{\mathrm{AGN} \text {,bol }}$, for PG quasars (filled black dots) and the AGN sample studied by $\mathrm{Zu}$ et al. (2011; open green dots).

surprising, given how closely the W4 and MIPS [24] bands resemble each other.

The MIPS-measured sample includes 33 of the $87 z<0.5$ PG quasars (plus 106 additional AGNs). To investigate the longerwavelength AGN IR variability for the complete PG sample, we compared their WISE W3 $(\sim 12 \mu \mathrm{m})$ and W4 $(\sim 22 \mu \mathrm{m})$ measurements and Spitzer/IRS spectra (Houck et al. 2004). We adopted the profile-fit photometry from the ALLWISE Source Catalog, whose measurements were carried out on the coadded image atlas, since little variability was detected in PG quasars at these wavelengths. Due to the wide passbands of $W 3$ and $W 4$, as well as the calibration uncertainty of the $W 4$ relative spectral response (Wright et al. 2010), we introduced flux corrections by increasing the $W 3$ band by $17 \%$ and decreasing the $W 4$ band by $10 \%$, based on the typical $f_{\nu} \sim \nu^{-1}$ SED shape of unobscured quasars at these wavelengths, as suggested by the Explanatory Supplement to the WISE All-Sky Data Release Products. ${ }^{6}$

\subsubsection{Synthesis Photometry from Spitzer/IRS Spectra}

To be compared with the measurements in the WISE W3 and W4 bands, we stitched the Spitzer/IRS spectra from different module observations together and computed synthetic photometry by convolving the spectral flux with the corresponding photometry relative spectral response curves. For the synthesis photometry uncertainty, we convolved the total error spectra, which include statistical and systematic errors, ${ }^{7}$ with the relative photometry curves.

\section{Reverberation Analysis Methods}

In contrast to the RM analysis of the optical emission lines, the transfer functions of the dust mid-IR response are greatly complicated by the radiation transfer of an IR optically thick torus, whose structures, density profile, grain properties, and inclination angle to the observer are poorly known (e.g., Kawaguchi \& Mori 2011; Almeyda et al. 2017). With the

\footnotetext{
6 See details in http://wise2.ipac.caltech.edu/docs/release/allsky/expsup/ sec4_4h.html.

7 See http://irs.sirtf.com/Smart/CassisProducts.
}

sparse cadence of the IR light curves, it is not very meaningful to fit these data with the simulated reverberation response of any specific torus model. Instead, we develop a "minimalist" method to retrieve the most important properties of the torus by comparing the IR and optical light curves.

\subsection{Constraining the Optical Continuum Variability}

The optical continuum variability of bright AGNs is well described by a DRW model (e.g., Kelly et al. 2009; Kozłowski et al. 2010; MacLeod et al. 2010; Zu et al. 2011). In this model, the quasar optical light curves can be reproduced as a stochastic process, and the covariance function follows $S(\Delta t)=$ $\sigma^{2} \exp \left(-\left|\Delta t / \tau_{\text {DRW }}\right|\right)$, where $\sigma$ is the amplitude and $\tau_{\text {DRW }}$ is the damping timescale. We use JAVELIN (Zu et al. 2013) to fit the optical light curves with the DRW model and fill the time gaps with the model values. ${ }^{8}$

The damping timescale $\tau_{\text {DRW }}$ is known to have correlations with AGN luminosity (Kelly et al. 2009; Zu et al. 2011). In Figure 4, we confirm such a correlation also exists by modeling our stitched optical light curves; the trend extends the work by $\mathrm{Zu}$ et al. (2011) to higher luminosity. The consistency demonstrated in Figure 4 shows that, despite their relatively low quality, the optical light curves compiled from these ground transient surveys should be good enough to produce meaningful results. In addition, the characteristic variability timescales, as traced by $\tau_{\mathrm{DRW}}$, are so long that averaging flux measurements and smoothing light curves (introduced later) would not introduce much in the way of systematic uncertainty. Finally, the optical variability timescales are typically larger than the $\sim 200$ days per epoch cadences of the mid-IR light curves; thus, the dust reverberation signals should be detected with the sparsely sampled WISE data.

\subsection{Retrieving Dust Reverberation Signals}

Traditional analyses of the dust reverberation signals were focused on calculating time lags between two light curves by cross-correlation. Although we use this method later to confirm the results, the low cadence of our data led us to develop an alternative method. In addition to the dust time lag, the scaling factor between the IR and optical variability amplitudes gives another important piece of information regarding the AGN torus. We therefore decided to introduce a simple model based only on single-value time lags and amplitudes to retrieve useful reverberation signals and mitigate the influences of many data quality issues.

As discussed in Appendix B, if the viewing angle is not perfectly face-on, the dust response lags on the same radius will have a range, resulting in a smoothing response to the optical variation features in the IR light curve (see also

\footnotetext{
8 The DRW model is also known as the simplest of the continuous-time autoregressive moving average process models (CARMA $(1,0)$ or CAR(1)). Despite its wide use, evidence for deviations from the CAR(1) model for AGN optical light curves has been found (e.g., Mushotzky et al. 2011; Graham et al. 2014), and some authors have suggested that higher-order CARMA models should be adopted (e.g., Kelly et al. 2014; Kasliwal et al. 2017). However, as demonstrated in Kasliwal et al. (2017), the behavior of the DRW model and more sophisticated CARMA models is identical for timescales longer than $\sim 10$ days. Our optical light-curve data typically have significant flux uncertainties, and their average time samplings are $\sim 5-10$ days. To be compared with the IR light curves (with time-sampling intervals of 200 days), the fitted optical light curves need be further smoothed (see later). As a result, it is not necessary to use higher-order CARMA models to describe the optical light curves.
} 
Kawaguchi \& Mori 2011). Therefore, for simplicity, we apply a top-hat function, $b(\tau)$, to smooth the optical DRW model fitting curve, $F(t)_{\mathrm{OPT}}$, so that

$$
\left\langle F(t)_{\mathrm{OPT}}\right\rangle_{\tau_{\mathrm{W}}}=\int_{-\tau_{\mathrm{W}} / 2}^{+\tau_{\mathrm{W}} / 2} F(t-\tau)_{\mathrm{OPT}} b(\tau) d \tau,
$$

where $\tau_{\mathrm{W}}$ is the width of the boxcar. On average, the smoothing window size should be correlated with the size of the dust emission structure, $R$, which is traced by the average time lag, $\Delta t$, between the IR and optical light curves. By default, the fitted optical DRW model is smoothed on-the-fly with $\tau_{\mathrm{W}}=\Delta t / 2$ and a maximum value of $\tau_{\mathrm{W}}=200$ days. This upper limit is set to be similar to the time gaps among the NEOWISE epochs, as well as in the optical light curve, to avoid oversmoothing.

We assume that, to first order, the IR dust emission light curve $F(t)_{\mathrm{IR}}$ can be described as a scaled version of the smoothed optical light curve $\left\langle F\left(t^{\prime}\right)_{\text {OPT }}\right\rangle$ with a constant time lag $\Delta t=t^{\prime}-t$,

$$
F(t)_{\mathrm{IR}, \text { dust }}=\mathrm{AMP} \times\left\langle F(t-\Delta t)_{\mathrm{OPT}}\right\rangle_{\tau_{\mathrm{W}}}+F_{\text {const. }},
$$

where AMP is the ratio between the optical and IR flux variation amplitudes, and $F_{\text {const }}$ is the systematic, timeinsensitive flux shift between the optical and IR bands. Physically, AMP reflects the efficiency of the dust IR energy transfer from the optical variation signal, which is related to the amount of dust; $\Delta t$ is the light travel time from the accretion disk to the dust torus at the studied wavelength. The constant, $F_{\text {const }}$, is determined by (1) the AGN SED averaged over a long period of time, (2) systemic uncertainties between the optical and mid-IR flux zero-points, and (3) contamination from the host galaxy emission or nearby sources.

The AGN accretion disk could also produce some variability in the mid-IR light curves. With spectropolarimetry observations of a small sample of bright quasars, Kishimoto et al. (2008) showed that the quasar accretion disk power-law continuum extends into the near-IR $(\lambda \sim 2 \mu \mathrm{m})$ and is consistent with an $F_{\nu} \propto \nu^{1 / 3}$ shape. It is not clear at what wavelength the accretion disk emission would transfer to a Rayleigh-Jeans slope; typically, a value of $3-5 \mu \mathrm{m}$ is suggested in the literature (e.g., Hönig et al. 2010; Stalevski et al. 2016). Considering the lack of constraints, we assume that the same $F_{\nu} \propto \nu^{1 / 3}$ spectral shape is valid to describe the accretion disk emission from the optical to the mid-IR bands. Due to the weak wavelength dependence of the accretion disk variability (e.g., Jiang et al. 2017), the accretion disk emission in the mid-IR can be assumed to change simultaneously with the optical. As a result, we have

$$
F(t)_{\mathrm{IR}, \text { accr. disk }}=F(t)_{\mathrm{OPT}}\left(\frac{\nu_{\mathrm{IR}}}{\nu_{\mathrm{OPT}}}\right)^{1 / 3},
$$

and $\nu_{\mathrm{IR}} / \nu_{\mathrm{OPT}} \sim 0.16$ and $\sim 0.12$ for the WISE W1 and W2 bands, respectively. To recover the dust reverberation signals, the contribution of IR variability by the accretion disk itself needs to be removed according to

$$
F(t)_{\mathrm{IR}, \text { dust }}=F(t)_{\mathrm{IR}}-F(t)_{\mathrm{IR}, \text { accr. disk }},
$$

where $F(t)_{\text {IR }}$ is the observed WISE light curve. As pointed out at the very end of Section 2.2, various time-insensitive contaminations and uncertainties in the optical light curves would only cause a flux offset. With our linear models to relate the optical and IR variability, these factors are included in $F_{\text {const }}$ and should not influence the measurements of $\Delta t$ and AMP.

Based on the model described above, we fitted the smoothed and delayed optical light curves (interpolated by the DRW model) to the WISE WI and W2 ones separately with the Levenberg-Marquardt least-squares fitting procedure as implemented in the IDL MPFIT (Markwardt 2009) package. Figure 5 provides some examples of how this approach applies to real data.

\subsection{Detecting the Time Lags}

Two methods have been adopted to find the most likely time lags. We first use the classical $\chi^{2}$ minimization technique to get best-fit values of AMP, $\Delta t$, and $F_{\text {const }}$ by fitting the rescaled and shifted optical DRW light-curve model to the WISE WI and W2 light curves separately. To explore the influences of different initial values on the final results, we change the initial guesses of $\Delta t /$ day from zero to 3000 with an increment of 10 . For objects with multiple local minimized $\chi^{2}$ values, the parameter values with the smallest $\chi^{2}$ were usually adopted in the end. For the time-lag uncertainty, the error provided by MPFIT only accounts for the measurement uncertainties of the mid-IR fluxes. To estimate the uncertainty introduced by the DRW interpolation of the optical light curve and the optical data themselves, we compute 1000 (optical) DRW models with the Monte Carlo (MC) method around the best-fit parameters of the optical light curves within the observing constraints from JAVELIN. Adopting the same initial values of the model parameters for the MPFIT code, the same observed mid-IR light curve is then repeatedly fitted by all 1000 mock optical DRW light curves with the same RM model using MPFIT. For the majority of objects, the distribution of the best-fit time lags with these optical mock light curves is symmetric and can be approximated by a Gaussian. We trim away outliers with $3 \sigma$ clipping and compute the standard deviation to represent the $1 \sigma$ uncertainty caused by the optical data and DRW model. The final time-lag uncertainty is a combination of this optical uncertainty based on MC simulation and the IR uncertainty reported by MPFIT.

After removing the accretion disk variability in the AGN IR emission following Equations (6) and (7), we also performed a cross-correlation analysis to constrain the time lags between the optical and the mid-IR light curves. For each object, the interpolated optical light curve based on the DRW model was shifted with $\Delta t=-300-3500$ days, and the cross-correlation functions $(\operatorname{CCF}(\Delta t))$ to the WISE mid-IR light curves were calculated. We provide several examples in Figure 6. In general, we adopt $\operatorname{CCF}(\Delta t)>0.8$ as the criterion for a strong correlation signal. Due to the limited time sampling, multiple $\operatorname{CCF}(\Delta)$ peaks for the optical-mid-IR light-curve pair can be found in many objects. In such cases, we inspect the peak locations sorted by the correlation values with the following steps: (1) we first reject any peaks close to the maximum of the explored $\Delta t$ range, which are nonphysical given the possible torus sizes for the AGN luminosity range; and then (2) we compare the other peak locations to the time lag derived from the $\chi^{2}$ fitting and adopt the one with the smallest discrepancy. For the vast majority of the sample, such a peak location has 


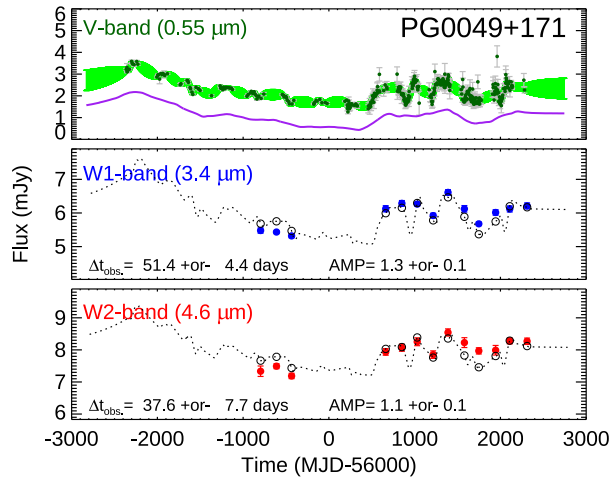

(a)

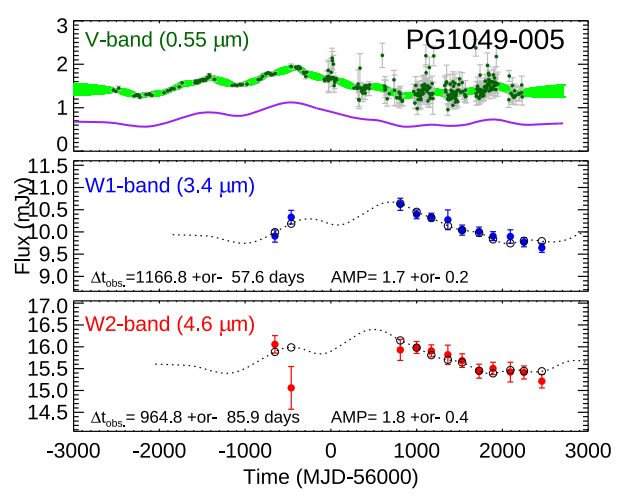

(d)

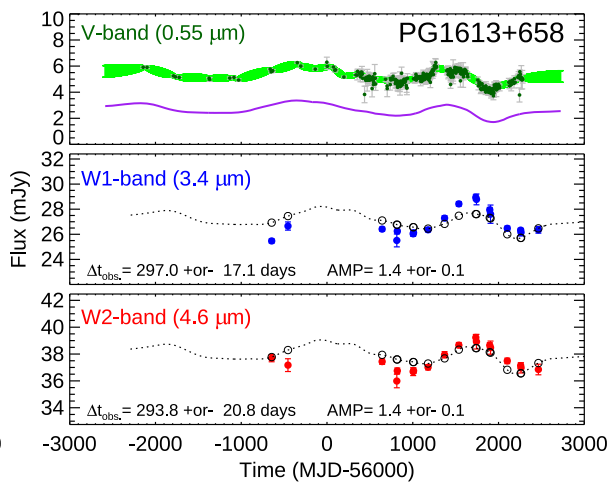

(b)

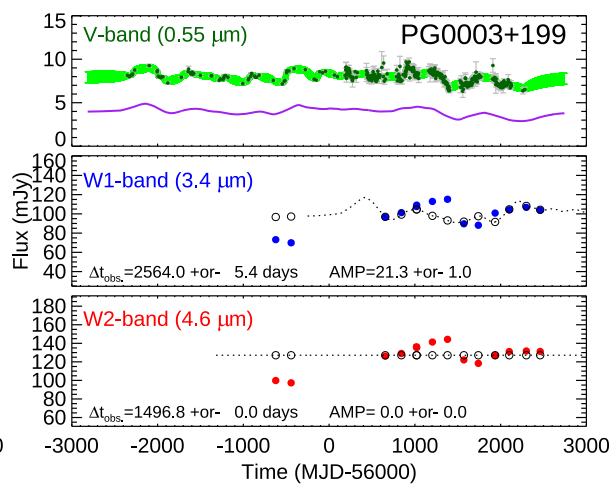

(e)

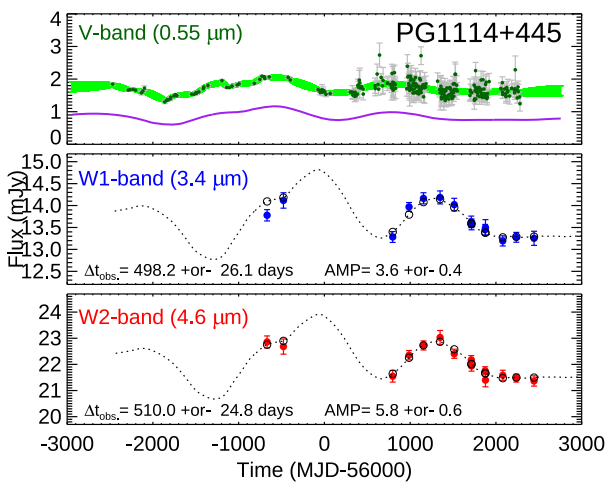

(c)

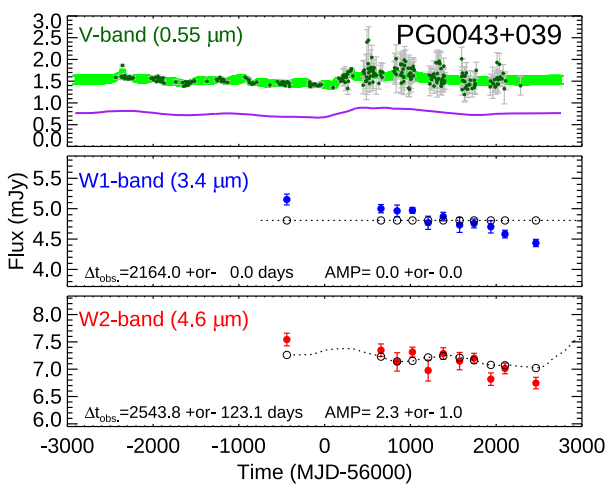

(f)

Figure 5. Representative optical and mid-IR light curves and corresponding DRW and time-lag fits of PG quasars. Objects in panels (a)-(d) have convincing dust reverberation signals with different mid-IR time lags from $\sim 60$ to $\sim 1400$ days, panel (e) gives an example whose IR light curves are uncorrelated with the optical ones, and panel (f) is a case where the mid-IR and/or the optical light curves do not contain enough features to get any convincing time-lag measurement. All measurements are presented in the observed frame. The optical (green), $3.4 \mu \mathrm{m}$ (blue), and $4.6 \mu \mathrm{m}$ (red) data are presented in the top, middle, and bottom subpanels, respectively. The thick green line in the top subpanel is a DRW model constrained by the optical light curve, with the solid purple line representing a smoothed version of the model scaled down for clarity. The dashed lines in the middle and bottom subpanels are the model based on the optical light curve to fit the IR, as described in Section 3.2. The model mid-IR fluxes at the observed epochs are shown as open circles. We denote the best-fit parameters with their errors for the mid-IR reverberation signals at the bottom of the corresponding subpanels.

(The complete figure set (87 images) is available.)

the maximum CCF value. In Figure 7, we compare the time lags from light-curve fitting with the maximum $\operatorname{CCF}(\Delta t)$ from cross-correlation analysis. In general, these two methods yield consistent $\Delta t$, particularly for the $W l$ band.

By default, the final time lags reported in Table 4 are adopted from the light-curve fitting method. However, in cases like PG 0049+171 where the MPFIT code returns suspicious results, e.g., $\Delta_{\mathrm{W} 1}>\Delta_{\mathrm{W} 2}$, we choose to adopt the time lags inferred from the cross-correlation analysis. The reasons for doing this is that model fittings with MPFIT are sensitive to the initial values and the fittings can be stopped at some local minimum of the parameter space. In addition, given the sparse sampling of the light curves, the solution is also not unique. We have inspected each object carefully to check if the fitting results make sense.

We can have a more intuitive idea on how the dust reverberation signals are retrieved by looking at representative objects individually in Figures 5 and 6. In both figures, panels (a)(d) present four examples with convincing measurements of timelags over a broad range, and panels (e) and (f) are two cases whose dust RM fittings are rejected. For objects with convincing time-lags, both optical and mid-IR light curves have clear features like small dents (panel (a)), bumps (panels (b) and (c)), or sometimes just a simple slope (panel (d)). For these objects, typically, there are clear peaks in their cross-correlation curve with
$\mathrm{CCF} \gtrsim 0.8$, and the corresponding time-lag is identical to that obtained by RM model fitting. Objects like the one in panel (e) are rejected because their optical and mid-IR light curves seem uncorrelated. As shown in panel (e) of Figure 5, both optical and mid-IR light curves have strong features, but the RM model fitting poorly reproduces the mid-IR data. For such objects, the peak of the CCF values are typically smaller than 0.6 , suggesting a weak, if nonzero, correlation. As will be argued in Section 5.1, there could be other explanations for the IR variability of such objects. Objects like panel (f) do not have enough features in the mid-IR light curves, and their optical light curves are also typically of very low variability amplitude. Thus, the data themselves cannot allow the model to determine the fitting parameters. In Figure 6, it can be seen that the cross-correlation analysis does not show any peaks for this source with $\mathrm{CCF} \gtrsim 0.5$ for any reasonable timelags (the Wl-band dust time-lags for quasars are very unlikely to have $\Delta t>2000$ days; see Section 4.2). We will reject such objects for the reverberation analysis.

\section{Results}

\subsection{Mid-IR Variability and Dust Reverberation Signals}

Table 4 summarizes the derived parameters from fitting the optical and WISE W1 and W2 mid-IR light curves with the RM 

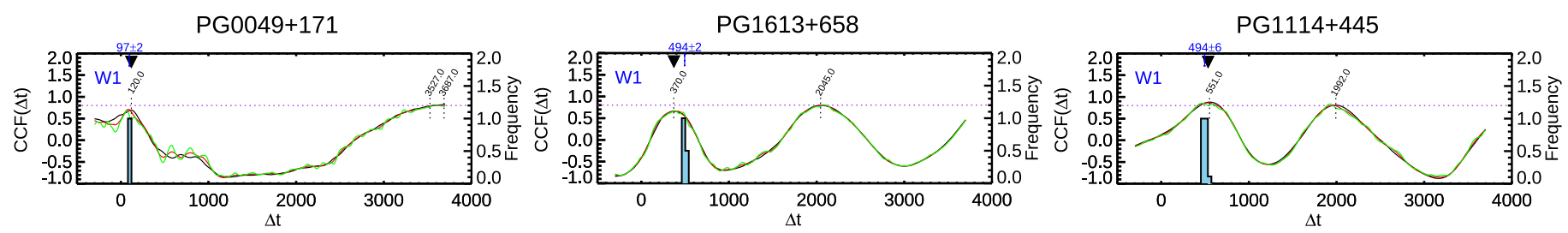

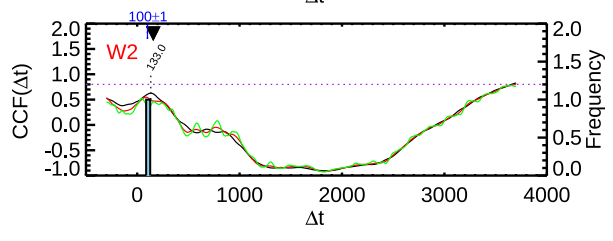

(a)
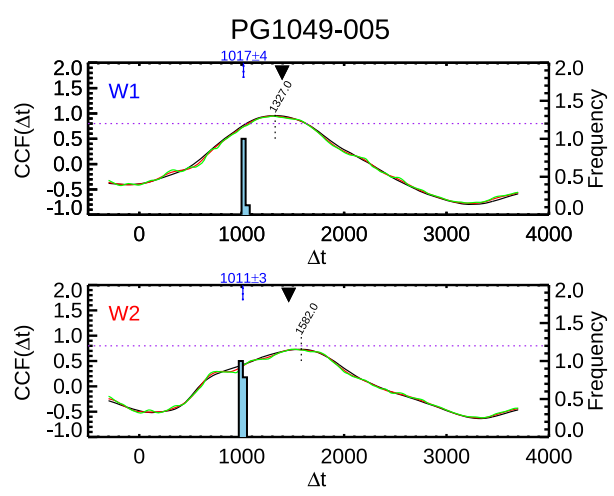

(d)

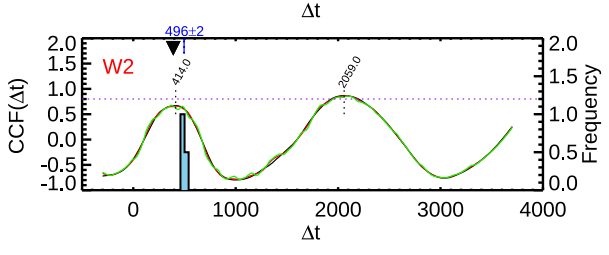

(b)
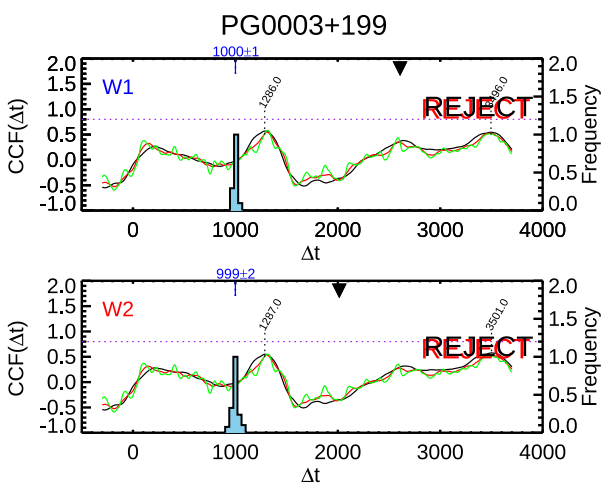

(e)

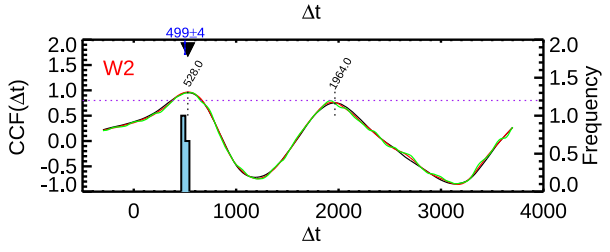

(c)

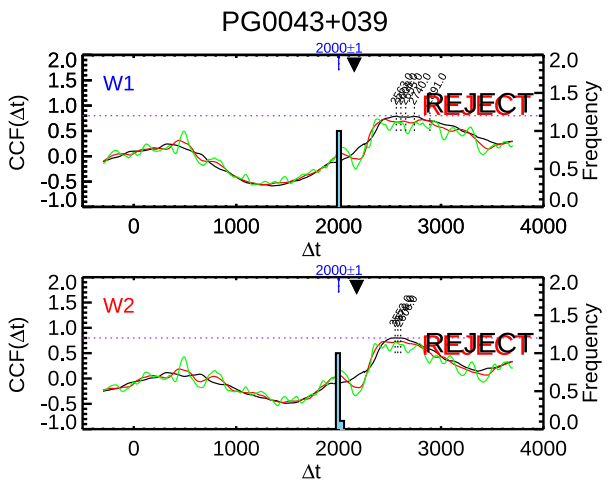

(f)

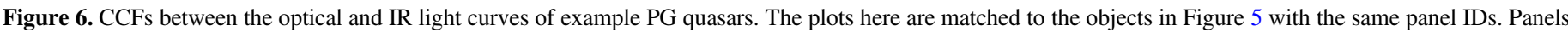

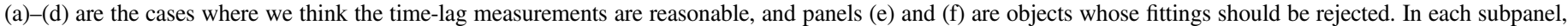

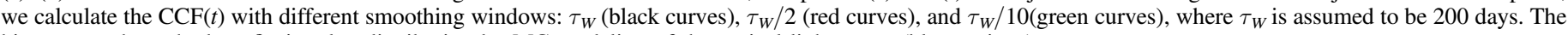
histograms show the best-fit time-lag distribution by MC modeling of the optical light curve (blue regions).

(The complete figure set ( 87 images) is available.)

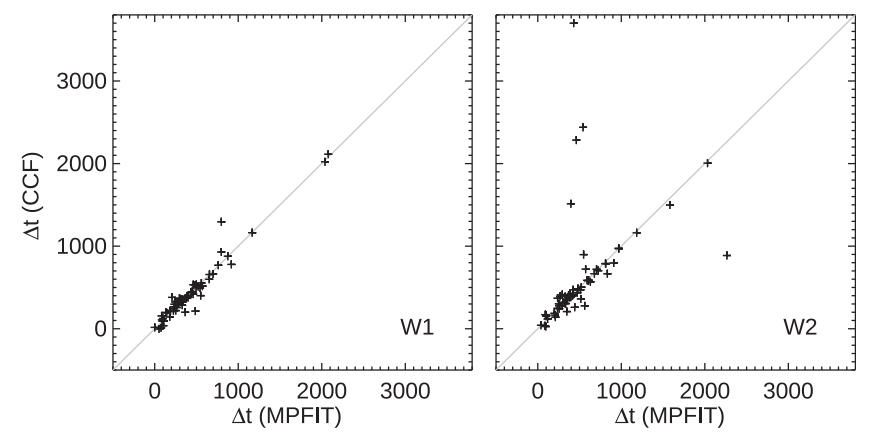

Figure 7. Comparison of mid-IR time-lags between the results from $\chi^{2}$ fittings and cross-correlation analysis. The gray diagonal lines are the 1:1 relation.

model introduced above. Among the 87 PG quasars, we detect convincing dust time-lag signals in 67 objects. For those objects without time-lag measurements, eight have data completeness or quality issues, eight lack clear variability features (e.g., very smooth light curves) to pin down a best-fit model (e.g., PG 0043+039, as shown in Figure 5), and the remaining four have uncorrelated optical and mid-IR temporal variations (e.g., PG 0003+199, as shown in Figure 5). In addition, there are nine quasars whose WISE flux during the first two or three epochs falls well above or below the timeshifted DRW model prediction in both bands, and we rely on the later NEOWISE epochs to extract the dust RM signals.
For the study of the time-lag-AGN luminosity relation, we will focus on the $67 \mathrm{PG}$ quasars ( $\sim 77 \%$ of the sample) with convincing time-lag measurements and assume that the torus properties are stable over timescales of several years or more.

\subsection{The IR Time-Lag-AGN Luminosity Correlation}

The inner size of the dust torus is physically determined by grain sublimation. Since carbon and silicate dust are the dominant species of interstellar grains, a similar grain mixture is also commonly assumed for the AGN torus. The sublimation temperatures, $T_{\text {sub }}$, of grains are estimated to be $1500-1800 \mathrm{~K}$ for graphite and 800-1000 K for silicates. Consequently, only graphite grains would survive at the innermost regions, and silicate grains would be distributed at larger radii. Assuming an optically thin environment, Barvainis (1987) provided an estimate of the graphite sublimation radius,

$$
\frac{R_{\text {sub }, \mathrm{C}}}{\mathrm{pc}}=1.3\left(\frac{L_{\mathrm{UV}}}{10^{46} \mathrm{erg} \mathrm{s}^{-1}}\right)^{0.5}\left(\frac{T_{\mathrm{sub}}}{1500 \mathrm{~K}}\right)^{-2.8}\left(\frac{a}{0.05 \mu \mathrm{m}}\right)^{-0.5},
$$

where $L_{\mathrm{UV}}$ is the AGN UV luminosity. Following Kishimoto et al. (2007), we also introduce an $a^{-1 / 2}$ term to approximate the $R_{\text {sub }}$ dependence on the grain size $a$. For silicate dust grains, adopting the absorption efficiency of astronomical silicate (Draine \& Lee 1984; Laor \& Draine 1993) and repeating the 
Table 4

Summary of the Mid-IR Reverberation Properties

\begin{tabular}{|c|c|c|c|c|c|c|c|c|c|c|c|c|c|}
\hline $\begin{array}{l}\text { Name } \\
\text { (1) }\end{array}$ & $\begin{array}{c}z \\
(2)\end{array}$ & $\begin{array}{l}\text { Type } \\
\text { (3) }\end{array}$ & $\begin{array}{l}L_{\mathrm{AGN}, \mathrm{bol}} \\
(4)\end{array}$ & $\begin{array}{c}\log _{e}(\sigma) \\
(5)\end{array}$ & $\begin{array}{c}\log _{e}(\tau) \\
(6)\end{array}$ & $\begin{array}{l}\Delta t_{W 1, \chi^{2}} \\
\quad \text { (7) }\end{array}$ & $\underset{(8)}{\operatorname{AMP}_{W 1}}$ & $\underset{(9)}{\Delta t_{W 1, \mathrm{CCF}}}$ & $\begin{array}{c}\Delta t_{W 2, \chi^{2}} \\
(10)\end{array}$ & $\begin{array}{c}\mathrm{AMP}_{W 2} \\
(11)\end{array}$ & $\underset{(12)}{\Delta t_{W 2, \mathrm{CCF}}}$ & $\begin{array}{c}\operatorname{Tag}_{W I} \\
(13)\end{array}$ & $\begin{array}{c}\mathrm{Tag}_{W 2} \\
\text { (14) }\end{array}$ \\
\hline PG $0003+158$ & 0.45 & HDD & 13.3 & -1.64 & 6.95 & $1084.1 \pm 402.0$ & $0.2 \pm 0.1$ & $3700(0.55)$ & $1057.5 \pm 462.0$ & $0.1 \pm 0.2$ & $3700(0.34)$ & 0 & 0 \\
\hline PG $0003+199$ & 0.03 & Norm & 11.2 & -0.42 & 5.43 & $2607.5 \pm 288.3$ & $20.0 \pm 1.0$ & $1287(0.57)$ & $2014.3 \pm 332.7$ & $0.0 \pm 0.0$ & $3501(0.58)$ & 0 & 0 \\
\hline PG $0007+106$ & 0.09 & Norm & 11.9 & -1.02 & 5.70 & $280.0 \pm 41.0$ & $4.7 \pm 0.2$ & $316(0.96)$ & $313.6 \pm 34.5$ & $4.5 \pm 0.2$ & $332(0.92)$ & 1 & 1 \\
\hline PG $0026+129$ & 0.14 & HDD & 12.4 & -1.04 & 6.53 & $557.2 \pm 41.4$ & $1.7 \pm 0.2$ & $503(0.91)$ & $624.9 \pm 33.7$ & $2.1 \pm 0.2$ & $1488(0.90)$ & 1 & 1 \\
\hline PG $0043+039$ & 0.38 & HDD? & 13.0 & -2.30 & 4.60 & $2152.8 \pm 187.5$ & $0.0 \pm 0.0$ & $2564(0.79)$ & $2176.2 \pm 264.7$ & $0.0 \pm 0.0$ & $2553(0.80)$ & 0 & 0 \\
\hline PG $0049+171$ & 0.06 & HDD & 11.6 & -0.60 & 6.45 & $120.0 \pm 11.4$ & $0.8 \pm 0.1$ & $3688(0.82)$ & $157.0 \pm 28.7$ & $0.6 \pm 0.1$ & $3700(0.83)$ & 1 & 1 \\
\hline PG $0050+124$ & 0.06 & Norm & 12.3 & -0.32 & 4.76 & $290.4 \pm 42.8$ & $14.9 \pm 0.4$ & $325(0.95)$ & $348.0 \pm 43.8$ & $15.2 \pm 0.5$ & $354(0.95)$ & 1 & 1 \\
\hline PG $0052+251$ & 0.16 & HDD & 12.6 & -0.94 & 6.52 & $400.5 \pm 42.9$ & $1.1 \pm 0.1$ & $2569(0.77)$ & $463.4 \pm 60.7$ & $0.9 \pm 0.1$ & $2555(0.81)$ & 1 & 1 \\
\hline PG $0157+001$ & 0.16 & Norm & 12.6 & -1.51 & 6.67 & $916.5 \pm 0.4$ & $17.8 \pm 0.5$ & $244(0.96)$ & $910.8 \pm 0.4$ & $17.3 \pm 0.7$ & $252(0.95)$ & 1 & 1 \\
\hline PG $0804+761$ & 0.10 & WDD & 12.6 & 0.11 & 6.68 & $659.9 \pm 22.7$ & $5.2 \pm 0.1$ & $665(0.92)$ & $661.2 \pm 23.8$ & $5.2 \pm 0.2$ & $676(0.92)$ & 1 & 1 \\
\hline PG $0838+770$ & 0.13 & Norm & 11.9 & -1.71 & 6.20 & $347.7 \pm 5.1$ & $5.0 \pm 0.1$ & $339(0.97)$ & $362.1 \pm 52.8$ & $5.8 \pm 0.2$ & $364(0.98)$ & 1 & 1 \\
\hline PG $0844+349$ & 0.06 & HDD? & 11.9 & -0.32 & 5.31 & $230.8 \pm 31.2$ & $1.5 \pm 0.1$ & $255(0.93)$ & $288.0 \pm 31.5$ & $1.3 \pm 0.1$ & $287(0.92)$ & 1 & 1 \\
\hline PG $0921+525$ & 0.04 & Norm & 11.0 & -0.00 & 5.18 & $101.8 \pm 3.0$ & $3.4 \pm 0.1$ & $159(0.85)$ & $104.0 \pm 3.3$ & $2.7 \pm 0.1$ & $187(0.82)$ & 1 & 1 \\
\hline PG $0923+201$ & 0.19 & WDD & 12.6 & -1.23 & 7.04 & $1200.8 \pm 92.4$ & $4.9 \pm 0.4$ & $1155(0.62)$ & $1327.8 \pm 151.5$ & $4.9 \pm 0.5$ & $1305(0.87)$ & 0 & 0 \\
\hline PG $0923+129$ & 0.03 & Norm & 11.0 & -1.06 & 4.12 & $306.1 \pm 11.9$ & $8.0 \pm 0.4$ & $86(0.81)$ & $298.8 \pm 16.5$ & $7.5 \pm 0.6$ & $1272(0.76)$ & 0 & 0 \\
\hline PG $0934+013$ & 0.05 & Norm & 10.9 & -1.48 & 6.34 & $330.4 \pm 10.3$ & $9.3 \pm 0.2$ & $291(0.97)$ & $367.9 \pm 68.8$ & $11.9 \pm 0.3$ & $482(0.96)$ & 1 & 1 \\
\hline PG $0947+396$ & 0.21 & Norm & 12.4 & -1.68 & 6.64 & $1963.5 \pm 28.2$ & $7.3 \pm 0.3$ & $1915(0.89)$ & $2021.3 \pm 100.4$ & $11.9 \pm 0.5$ & $1915(0.84)$ & 0 & 0 \\
\hline PG $0953+414$ & 0.24 & WDD & 12.9 & -0.42 & 7.30 & $913.0 \pm 35.6$ & $2.8 \pm 0.1$ & $911(0.97)$ & $1153.6 \pm 49.9$ & $2.1 \pm 0.2$ & $1134(0.98)$ & 1 & 1 \\
\hline PG $1001+054$ & 0.16 & Norm & 12.0 & -1.60 & 6.39 & $256.0 \pm 47.9$ & $6.3 \pm 0.4$ & $715(0.86)$ & $255.0 \pm 91.4$ & $9.4 \pm 0.8$ & $370(0.92)$ & 1 & 1 \\
\hline PG $1004+130$ & 0.24 & Norm & 12.6 & -1.30 & 6.80 & $796.0 \pm 93.7$ & $1.6 \pm 0.2$ & $707(0.83)$ & $532.0 \pm 107.8$ & $0.9 \pm 0.1$ & $692(0.68)$ & 1 & 1 \\
\hline PG $1011-040$ & 0.06 & HDD & 11.7 & -1.26 & 6.87 & $424.8 \pm 35.9$ & $3.0 \pm 0.2$ & $415(0.96)$ & $510.8 \pm 47.7$ & $3.0 \pm 0.2$ & $480(0.95)$ & 1 & 1 \\
\hline PG $1012+008$ & 0.19 & Norm & 12.2 & -1.67 & 6.51 & $519.8 \pm 20.1$ & $5.8 \pm 0.2$ & $536(0.98)$ & $570.5 \pm 94.3$ & $5.4 \pm 0.3$ & $2463(0.97)$ & 1 & 1 \\
\hline PG $1022+519$ & 0.05 & HDD? & 11.2 & -1.78 & 5.23 & $94.0 \pm 47.5$ & $6.6 \pm 0.2$ & $94(0.93)$ & $118.0 \pm 43.5$ & $7.2 \pm 0.3$ & $125(0.94)$ & 1 & 1 \\
\hline PG $1048+342$ & 0.17 & Norm & 11.8 & -2.14 & 6.94 & $276.0 \pm 61.5$ & $2.1 \pm 0.1$ & $241(0.87)$ & $565.8 \pm 115.5$ & $2.9 \pm 0.2$ & $572(0.85)$ & 1 & 1 \\
\hline PG $1048-090$ & 0.34 & WDD & 12.8 & -1.29 & 6.78 & $589.0 \pm 155.2$ & $0.0 \pm 0.0$ & $2632(0.54)$ & $549.7 \pm 55.3$ & $0.0 \pm 0.0$ & $2488(0.71)$ & 0 & 0 \\
\hline PG 1049-005 & 0.36 & Norm & 13.0 & -1.71 & 7.08 & $1284.6 \pm 108.3$ & $1.6 \pm 0.2$ & $1327(0.96)$ & $1059.3 \pm 130.8$ & $1.7 \pm 0.4$ & $1583(0.73)$ & 1 & 0 \\
\hline PG $1100+772$ & 0.31 & HDD & 13.1 & -1.38 & 6.13 & $720.8 \pm 88.1$ & $1.3 \pm 0.1$ & $722(0.59)$ & $724.4 \pm 144.8$ & $1.6 \pm 0.2$ & $725(0.58)$ & 0 & 0 \\
\hline PG $1103-006$ & 0.43 & Norm & 12.8 & -1.80 & 6.38 & $593.9 \pm 51.1$ & $0.6 \pm 0.2$ & $2223(0.46)$ & $823.0 \pm 273.4$ & $0.8 \pm 0.3$ & $2371(0.31)$ & 0 & 0 \\
\hline PG $1114+445$ & 0.14 & Norm & 12.3 & -1.73 & 6.55 & $534.8 \pm 21.7$ & $3.3 \pm 0.4$ & $551(0.88)$ & $527.9 \pm 37.0$ & $5.4 \pm 0.6$ & $528(0.96)$ & 1 & 1 \\
\hline PG $1115+407$ & 0.15 & HDD & 12.4 & -1.93 & 5.83 & $465.9 \pm 18.3$ & $5.0 \pm 0.3$ & $422(0.83)$ & $455.4 \pm 30.7$ & $4.3 \pm 0.5$ & $273(0.74)$ & 1 & 1 \\
\hline PG $1116+215$ & 0.18 & WDD & 12.9 & -0.40 & 7.28 & $819.6 \pm 98.6$ & $4.6 \pm 0.2$ & $1895(0.85)$ & $967.4 \pm 90.0$ & $4.9 \pm 0.3$ & $1139(0.83)$ & 1 & 1 \\
\hline PG $1119+120$ & 0.05 & Norm & 11.5 & -0.69 & 5.92 & $108.0 \pm 6.7$ & $3.1 \pm 0.2$ & $42(0.94)$ & $101.0 \pm 6.7$ & $2.7 \pm 0.2$ & $29(0.91)$ & 1 & 1 \\
\hline PG $1121+422$ & 0.23 & HDD & 12.5 & -1.48 & 7.28 & $912.1 \pm 116.1$ & $2.8 \pm 0.1$ & $497(0.94)$ & $515.2 \pm 82.4$ & $3.2 \pm 0.2$ & $2277(0.88)$ & 1 & 1 \\
\hline PG $1126-041$ & 0.06 & Norm & 11.8 & -0.41 & 6.27 & $554.1 \pm 28.3$ & $7.0 \pm 0.3$ & $566(0.87)$ & $661.1 \pm 25.4$ & $7.3 \pm 0.4$ & $731(0.88)$ & 1 & 1 \\
\hline PG $1149-110$ & 0.05 & Norm & 11.1 & -1.38 & 5.15 & $86.9 \pm 23.1$ & $7.6 \pm 0.2$ & $110(0.85)$ & $211.6 \pm 23.2$ & $7.6 \pm 0.2$ & $143(0.79)$ & 1 & 1 \\
\hline PG $1151+117$ & 0.18 & Norm & 12.0 & -1.25 & 7.69 & $295.9 \pm 27.3$ & $2.4 \pm 0.1$ & $341(0.96)$ & $414.0 \pm 63.5$ & $2.3 \pm 0.2$ & $414(0.87)$ & 1 & 1 \\
\hline PG $1202+281$ & 0.17 & Norm & 12.1 & -1.69 & 6.93 & $361.0 \pm 39.3$ & $7.8 \pm 0.4$ & $358(0.96)$ & $439.1 \pm 44.5$ & $7.9 \pm 0.5$ & $429(0.95)$ & 1 & 1 \\
\hline PG $1211+143$ & 0.09 & Norm & 12.1 & -0.07 & 4.79 & $365.6 \pm 90.2$ & $2.7 \pm 0.2$ & $358(0.88)$ & $557.5 \pm 47.6$ & $3.3 \pm 0.3$ & $2015(0.77)$ & 1 & 1 \\
\hline PG $1216+069$ & 0.33 & HDD & 13.0 & -0.10 & 3.23 & $1016.9 \pm 23.9$ & $0.3 \pm 0.1$ & $985(0.30)$ & $1012.9 \pm 76.3$ & $0.2 \pm 0.1$ & $703(0.34)$ & 0 & 0 \\
\hline PG $1226+023$ & 0.16 & WDD & 13.4 & 1.09 & 7.21 & $1918.4 \pm 67.9$ & $0.0 \pm 0.0$ & $933(0.75)$ & $1684.4 \pm 280.6$ & $0.0 \pm 0.0$ & $951(0.59)$ & 0 & 0 \\
\hline PG $1229+204$ & 0.06 & Norm & 11.5 & -0.70 & 6.87 & $266.3 \pm 71.2$ & $5.3 \pm 0.1$ & $261(0.98)$ & $328.0 \pm 8.5$ & $5.2 \pm 0.1$ & $305(0.97)$ & 1 & 1 \\
\hline PG $1244+026$ & 0.05 & Norm & 11.1 & -2.36 & 5.27 & $118.9 \pm 70.3$ & $0.8 \pm 0.3$ & $1101(0.60)$ & $180.0 \pm 177.3$ & $0.7 \pm 0.5$ & $1205(0.46)$ & 0 & 0 \\
\hline PG $1259+593$ & 0.47 & WDD & 13.3 & -1.54 & 7.25 & $746.2 \pm 55.9$ & $2.3 \pm 0.1$ & $732(0.98)$ & $784.4 \pm 70.3$ & $2.2 \pm 0.2$ & $794(0.93)$ & 1 & 1 \\
\hline PG $1302-102$ & 0.29 & HDD & 13.2 & -1.18 & 7.05 & $643.8 \pm 33.2$ & $1.6 \pm 0.1$ & $619(0.90)$ & $681.0 \pm 82.6$ & $1.8 \pm 0.2$ & $662(0.91)$ & 1 & 1 \\
\hline PG $1307+085$ & 0.16 & Norm & 12.2 & -1.51 & 6.20 & $357.6 \pm 46.1$ & $2.8 \pm 0.2$ & $334(0.91)$ & $505.9 \pm 57.7$ & $3.1 \pm 0.2$ & $368(0.87)$ & 1 & 1 \\
\hline PG $1309+355$ & 0.18 & Norm & 12.4 & -1.74 & 6.59 & $404.6 \pm 62.0$ & $2.5 \pm 0.3$ & $419(0.78)$ & $531.8 \pm 92.0$ & $1.6 \pm 0.5$ & $1453(0.58)$ & 1 & 1 \\
\hline PG $1310-108$ & 0.04 & Norm & 10.9 & -1.68 & 4.56 & $152.0 \pm 30.2$ & $2.0 \pm 0.3$ & $208(0.76)$ & $363.9 \pm 26.5$ & $4.4 \pm 0.4$ & $391(0.83)$ & 1 & 1 \\
\hline PG $1322+659$ & 0.17 & Norm & 12.1 & -1.29 & 6.38 & $524.2 \pm 54.3$ & $2.8 \pm 0.1$ & $657(0.89)$ & $745.0 \pm 115.0$ & $3.0 \pm 0.1$ & $748(0.86)$ & 1 & 1 \\
\hline PG $1341+258$ & 0.09 & HDD? & 11.7 & -2.25 & 5.27 & $351.1 \pm 246.1$ & $5.8 \pm 0.3$ & $356(0.71)$ & $383.9 \pm 18.5$ & $6.9 \pm 0.4$ & $383(0.71)$ & 1 & 1 \\
\hline PG $1351+236$ & 0.05 & Norm & 10.8 & -2.08 & 4.70 & $79.6 \pm 48.9$ & $7.9 \pm 0.4$ & $11(0.70)$ & $83.8 \pm 32.9$ & $5.1 \pm 0.4$ & $38(0.60)$ & 1 & 1 \\
\hline PG $1351+640$ & 0.09 & Norm & 12.2 & -0.37 & 6.11 & $631.2 \pm 29.5$ & $1.1 \pm 0.1$ & $1509(0.87)$ & $808.6 \pm 51.8$ & $1.5 \pm 0.2$ & $953(0.88)$ & 1 & 1 \\
\hline PG $1352+183$ & 0.16 & Norm & 11.9 & -1.70 & 6.20 & $272.0 \pm 10.1$ & $3.1 \pm 0.2$ & $247(0.91)$ & $316.0 \pm 78.4$ & $3.5 \pm 0.3$ & $329(0.94)$ & 1 & 1 \\
\hline PG $1354+213$ & 0.30 & Norm & 12.4 & -2.04 & 7.06 & $911.5 \pm 124.1$ & $4.4 \pm 0.2$ & $897(0.91)$ & $1061.4 \pm 169.3$ & $5.2 \pm 0.3$ & $1066(0.88)$ & 0 & 0 \\
\hline
\end{tabular}


Table 4

(Continued)

\begin{tabular}{|c|c|c|c|c|c|c|c|c|c|c|c|c|c|}
\hline $\begin{array}{l}\text { Name } \\
\text { (1) }\end{array}$ & $\begin{array}{c}z \\
(2)\end{array}$ & $\begin{array}{l}\text { Type } \\
\text { (3) }\end{array}$ & $\underset{(4)}{L_{\mathrm{AGN}, \text { bol }}}$ & $\underset{(5)}{\log _{e}(\sigma)}$ & $\begin{array}{c}\log _{e}(\tau) \\
\quad(6)\end{array}$ & $\begin{array}{c}\Delta t_{W 1, \chi^{2}} \\
\text { (7) }\end{array}$ & $\underset{(8)}{\operatorname{AMP}_{W 1}}$ & $\underset{(9)}{\Delta t_{W 1, \mathrm{CCF}}}$ & $\begin{array}{c}\Delta t_{W 2, \chi^{2}} \\
(10)\end{array}$ & $\underset{(11)}{\operatorname{AMP}_{W 2}}$ & $\frac{\Delta t_{W 2, \mathrm{CCF}}}{(12)}$ & $\begin{array}{c}\operatorname{Tag}_{W I} \\
\text { (13) }\end{array}$ & $\begin{array}{c}\mathrm{Tag}_{W 2} \\
\text { (14) }\end{array}$ \\
\hline PG $1402+261$ & 0.16 & Norm & 12.4 & -1.44 & 6.18 & $368.1 \pm 54.6$ & $5.6 \pm 0.4$ & $1972(0.91)$ & $414.6 \pm 82.4$ & $8.8 \pm 0.6$ & $2011(0.88)$ & 1 & 1 \\
\hline PG $1404+226$ & 0.10 & HDD? & 11.7 & -2.16 & 5.63 & $214.7 \pm 13.2$ & $2.4 \pm 0.2$ & $1893(0.86)$ & $272.0 \pm 30.7$ & $2.9 \pm 0.4$ & $1977(0.89)$ & 1 & 1 \\
\hline PG $1411+442$ & 0.09 & Norm & 12.0 & -0.55 & 6.89 & $441.7 \pm 46.2$ & $2.3 \pm 0.1$ & $704(0.88)$ & $445.2 \pm 77.6$ & $2.5 \pm 0.2$ & $1000(0.84)$ & 1 & 1 \\
\hline PG $1415+451$ & 0.11 & Norm & 11.7 & -1.79 & 6.64 & $300.0 \pm 232.3$ & $4.3 \pm 0.1$ & $350(0.95)$ & $677.4 \pm 245.2$ & $0.0 \pm 0.0$ & $433(0.91)$ & 1 & 1 \\
\hline PG $1416-129$ & 0.13 & Norm & 11.6 & -2.32 & 6.41 & $424.8 \pm 426.7$ & $2.8 \pm 0.3$ & $-162(0.53)$ & $511.0 \pm 113.4$ & $1.9 \pm 0.3$ & $530(0.44)$ & 0 & 0 \\
\hline PG $1425+267$ & 0.37 & Norm & 12.7 & -2.00 & 7.25 & $744.1 \pm 99.5$ & $0.8 \pm 0.1$ & $749(0.88)$ & $760.5 \pm 321.7$ & $0.6 \pm 0.2$ & $738(0.70)$ & 1 & 1 \\
\hline PG $1426+015$ & 0.09 & Norm & 12.0 & -0.11 & 6.77 & $264.0 \pm 24.7$ & $3.9 \pm 0.2$ & $314(0.95)$ & $352.0 \pm 64.5$ & $3.3 \pm 0.2$ & $359(0.92)$ & 1 & 1 \\
\hline PG $1427+480$ & 0.22 & Norm & 12.2 & -2.19 & 5.97 & $342.6 \pm 46.7$ & $1.4 \pm 0.1$ & $332(0.86)$ & $356.0 \pm 52.0$ & $1.1 \pm 0.2$ & $352(0.78)$ & 1 & 1 \\
\hline PG $1435-067$ & 0.13 & HDD & 12.2 & -1.88 & 5.43 & $244.0 \pm 42.1$ & $4.8 \pm 0.2$ & $314(0.86)$ & $356.1 \pm 63.6$ & $4.5 \pm 0.4$ & $362(0.82)$ & 1 & 1 \\
\hline PG $1440+356$ & 0.08 & Norm & 11.9 & -0.35 & 6.29 & $255.3 \pm 40.0$ & $4.3 \pm 0.2$ & $290(0.95)$ & $264.9 \pm 30.4$ & $4.7 \pm 0.2$ & $296(0.92)$ & 1 & 1 \\
\hline PG $1444+407$ & 0.27 & Norm & 12.7 & -1.95 & 6.52 & $399.5 \pm 16.4$ & $2.6 \pm 0.2$ & $405(0.93)$ & $448.1 \pm 61.1$ & $2.6 \pm 0.3$ & $505(0.74)$ & 1 & 1 \\
\hline PG $1448+273$ & 0.06 & Norm & 11.4 & -0.75 & 5.88 & $280.0 \pm 31.7$ & $2.8 \pm 0.1$ & $289(0.98)$ & $371.3 \pm 49.0$ & $2.6 \pm 0.1$ & $378(0.97)$ & 1 & 1 \\
\hline PG $1501+106$ & 0.04 & Norm & 11.4 & -0.05 & 5.91 & $113.9 \pm 16.4$ & $2.1 \pm 0.1$ & $151(0.85)$ & $112.0 \pm 11.9$ & $2.0 \pm 0.1$ & $2703(0.77)$ & 1 & 1 \\
\hline PG $1512+370$ & 0.37 & Norm & 12.7 & -1.95 & 6.91 & $1352.4 \pm 257.2$ & $0.5 \pm 0.1$ & $3152(0.82)$ & $2122.8 \pm 478.4$ & $1.2 \pm 0.3$ & $3383(0.68)$ & 0 & 0 \\
\hline PG $1519+226$ & 0.14 & Norm & 12.0 & -1.68 & 7.40 & $193.8 \pm 113.1$ & $7.4 \pm 0.2$ & $228(0.99)$ & $356.5 \pm 105.0$ & $6.2 \pm 0.3$ & $365(0.98)$ & 1 & 1 \\
\hline PG $1534+580$ & 0.03 & Norm & 10.9 & -0.67 & 5.08 & $128.0 \pm 2.9$ & $3.2 \pm 0.2$ & $240(0.67)$ & $139.3 \pm 12.7$ & $4.1 \pm 0.1$ & $376(0.75)$ & 0 & 0 \\
\hline PG $1535+547$ & 0.04 & Norm & 10.9 & -0.54 & 5.99 & $180.0 \pm 1.4$ & $8.5 \pm 0.1$ & $157(0.92)$ & $207.7 \pm 0.2$ & $7.7 \pm 0.2$ & $219(0.92)$ & 1 & 1 \\
\hline PG $1543+489$ & 0.40 & Norm & 13.1 & -2.88 & 5.85 & $2052.2 \pm 606.7$ & $8.9 \pm 0.9$ & $2002(0.74)$ & $2066.0 \pm 276.3$ & $13.4 \pm 1.3$ & $1974(0.72)$ & 1 & 1 \\
\hline PG $1545+210$ & 0.27 & WDD & 12.7 & -1.37 & 6.90 & $774.8 \pm 51.3$ & $1.8 \pm 0.1$ & $794(0.94)$ & $835.3 \pm 74.3$ & $2.1 \pm 0.1$ & $804(0.94)$ & 1 & 1 \\
\hline PG $1552+085$ & 0.12 & HDD? & 12.1 & -1.37 & 6.85 & $212.0 \pm 30.2$ & $3.3 \pm 0.1$ & $243(0.98)$ & $264.0 \pm 22.9$ & $3.5 \pm 0.1$ & $261(0.98)$ & 1 & 1 \\
\hline PG $1612+261$ & 0.13 & Norm & 12.0 & -0.98 & 6.77 & $627.3 \pm 39.7$ & $1.2 \pm 0.2$ & $624(0.41)$ & $664.2 \pm 62.0$ & $2.0 \pm 0.3$ & $1262(0.71)$ & 0 & 0 \\
\hline PG $1613+658$ & 0.13 & Norm & 12.4 & -0.65 & 6.43 & $371.9 \pm 63.3$ & $1.6 \pm 0.2$ & $2046(0.80)$ & $390.4 \pm 37.1$ & $1.8 \pm 0.2$ & $2059(0.87)$ & 1 & 1 \\
\hline PG $1617+175$ & 0.11 & WDD & 12.1 & -0.71 & 6.75 & $472.4 \pm 34.7$ & $3.3 \pm 0.1$ & $1187(0.97)$ & $575.8 \pm 26.7$ & $4.4 \pm 0.2$ & $1183(0.97)$ & 1 & 1 \\
\hline PG $1626+554$ & 0.13 & HDD & 12.2 & -1.14 & 6.10 & $346.8 \pm 7.4$ & $1.6 \pm 0.1$ & $381(0.90)$ & $414.5 \pm 9.2$ & $1.4 \pm 0.1$ & $416(0.81)$ & 1 & 1 \\
\hline PG $1700+518$ & 0.28 & Norm & 13.1 & -1.69 & 7.49 & $292.4 \pm 669.7$ & $1.4 \pm 0.3$ & $-300(0.80)$ & $967.9 \pm 216.2$ & $0.0 \pm 0.0$ & $3700(0.68)$ & 0 & 0 \\
\hline PG $1704+608$ & 0.37 & Norm & 13.1 & -1.15 & 6.93 & $1650.3 \pm 744.1$ & $0.7 \pm 0.1$ & $3700(0.60)$ & $1689.5 \pm 815.7$ & $0.8 \pm 0.1$ & $1660(0.70)$ & 0 & 0 \\
\hline PG $2112+059$ & 0.47 & Norm & 13.3 & -1.44 & 7.74 & $2265.0 \pm 105.9$ & $0.9 \pm 0.2$ & $646(0.95)$ & $2614.9 \pm 413.5$ & $1.1 \pm 1.0$ & $883(0.67)$ & 1 & 1 \\
\hline PG 2130+099 & 0.06 & Norm & 11.9 & -0.27 & 6.41 & $525.3 \pm 44.7$ & $5.8 \pm 0.2$ & $521(0.89)$ & $801.3 \pm 46.6$ & $5.5 \pm 0.2$ & $1075(0.89)$ & 1 & 1 \\
\hline PG $2209+184$ & 0.07 & HDD? & 11.6 & -1.02 & 6.23 & $108.0 \pm 10.4$ & $5.3 \pm 0.1$ & $19(0.95)$ & $187.0 \pm 13.3$ & $7.7 \pm 0.2$ & $189(0.97)$ & 1 & 1 \\
\hline PG $2214+139$ & 0.07 & WDD & 11.9 & -0.59 & 6.13 & $324.6 \pm 165.6$ & $4.2 \pm 0.4$ & $2545(0.86)$ & $378.4 \pm 622.9$ & $3.0 \pm 0.4$ & $2622(0.85)$ & 1 & 0 \\
\hline PG $2233+134$ & 0.32 & Norm & 12.7 & -2.30 & 6.73 & $455.1 \pm 39.0$ & $2.2 \pm 0.2$ & $449(0.90)$ & $426.9 \pm 47.7$ & $2.7 \pm 0.4$ & $420(0.83)$ & 1 & 1 \\
\hline PG $2251+113$ & 0.32 & WDD & 12.9 & -2.40 & 6.30 & $579.7 \pm 116.8$ & $2.7 \pm 0.8$ & $576(0.85)$ & $598.0 \pm 101.8$ & $3.5 \pm 1.0$ & $591(0.91)$ & 0 & 0 \\
\hline PG 2304+042 & 0.04 & Norm & 10.6 & 0.11 & 2.66 & $86.0 \pm 3.6$ & $3.7 \pm 0.0$ & $156(0.97)$ & $98.2 \pm 1.5$ & $3.8 \pm 0.1$ & $171(0.97)$ & 1 & 1 \\
\hline PG 2308+098 & 0.43 & HDD & 13.2 & -1.11 & 7.68 & $703.1 \pm 50.7$ & $1.3 \pm 0.1$ & $548(0.88)$ & $1418.8 \pm 129.5$ & $1.2 \pm 0.1$ & $1493(0.83)$ & 0 & 0 \\
\hline
\end{tabular}

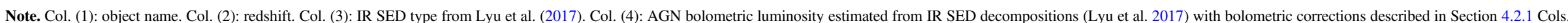

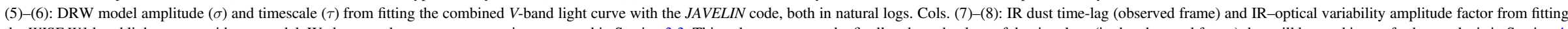

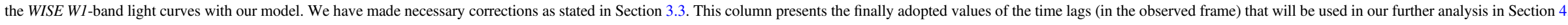

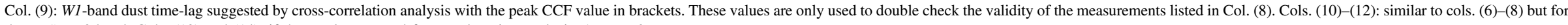
the WISE W2 band. Cols. (13) and (14): if the results are used for reverberation analysis, 1 -yes, 0 -no.

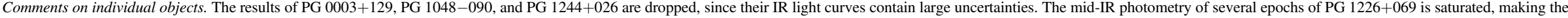

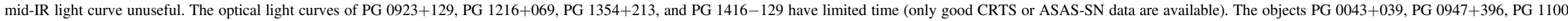

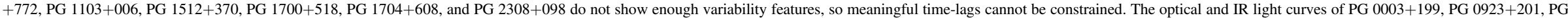

$1534+580$, and PG $1612+261$ seem uncorrelated with peak $\mathrm{CCF}<0.7$, so their results have also been dropped. 
derivations in Barvainis (1987), we have

$$
\frac{R_{\mathrm{sub}, \mathrm{S}}}{\mathrm{pc}}=2.7\left(\frac{L_{\mathrm{UV}}}{10^{46} \mathrm{erg} \mathrm{s}^{-1}}\right)^{0.5}\left(\frac{T_{\mathrm{sub}}}{1000 \mathrm{~K}}\right)^{-2.8}\left(\frac{a}{0.05 \mu \mathrm{m}}\right)^{-0.5} .
$$

If we take $L_{\mathrm{UV}}=0.165 L_{\mathrm{AGN}, \text { bol }}$ (Risaliti \& Elvis 2004) and assume $T_{\text {sub }}=1500$ and $1000 \mathrm{~K}$ for graphite and silicate dust grains, for an AGN with $L_{\mathrm{AGN}, \text { bol }}=10^{12} L_{\odot}, R_{\text {sub,C }} \sim 0.33 \mathrm{pc}$ and $R_{\text {sub }, \mathrm{S}} \sim 0.69 \mathrm{pc}$.

For an infinitely thin ring viewed from a perfect face-on observing angle, the time-lag is directly related to the dust radius as $\Delta t=R_{\mathrm{d}} / c$. In real situations, the relation would be dependent on the observing angle, as well as the dust distribution (some additional discussion is provided in Appendix B). However, for a large sample of type-1 AGNs, these effects would be smeared out. To first order, we adopt the following linear equation to fit the data:

$$
\log (\Delta t)=\alpha+\beta \log \left(L_{\mathrm{AGN}}\right) .
$$

In the following analysis and discussion, we have applied the dilation factor $(1+z)$ for the time-lag (i.e., $\Delta t=\Delta t_{\text {int. }}=$ $\left.\Delta t_{\text {obs. }} /(1+z)\right)$. Due to the lack of constraints on the wavelength-dependent torus size, we will not make $K$ corrections for individual quasars but treat the sample as a whole and compute the correction with an averaged redshift.

\subsubsection{AGN Luminosity Estimation}

To make a comparison of the AGN bolometric luminosity $L_{\mathrm{AGN}, \text { bol }}$ in the literature, we use the integrated AGN IR emission of PG quasars as the best estimator. As demonstrated in Lyu et al. (2017), despite their identical UV-optical SED shape, unobscured type-1 quasars present intrinsic SED variations in the IR that can be grouped into normal, warmdust-deficient (WDD) and hot-dust-deficient (HDD) types (see the comparison in Figure 8). After building the intrinsic AGN templates, Lyu et al. (2017) fit the observed SEDs of PG quasars with an empirical SED model. The AGN intrinsic SED types were determined by comparing the fitted $\chi^{2}$ of the model. We convert the AGN-heated IR luminosity derived from the optical-to-IR SED fittings in Lyu et al. (2017) to the monochromatic luminosity at $5100 \AA$ with the following scaling factors:

$$
\begin{gathered}
\lambda L_{\lambda}(0.51 \mu \mathrm{m})=0.47 L_{\mathrm{NORM}, 8-1000 \mu \mathrm{m}} \\
=1.02 L_{\mathrm{WDD}, 8-1000 \mu \mathrm{m}} \\
=1.75 L_{\mathrm{HDD}, 8-1000 \mu \mathrm{m},}
\end{gathered}
$$

where NORM, WDD, HDD represent the normal Elvis et al. (1994)-like, WDD, and HDD AGN templates as characterized in Lyu et al. (2017) and Lyu \& Rieke (2017). We then convert $L_{\lambda}(0.51 \mu \mathrm{m})$ to $L_{\mathrm{AGN}, \text { bol }}$ with the updated quasar bolometric correction from Runnoe et al. (2012):

$$
\log \left(\frac{L_{\mathrm{AGN}, \mathrm{bol}}}{\mathrm{erg} \mathrm{s}^{-1}}\right)=4.89+0.91 \log \left(\frac{\lambda L_{\lambda}(0.51 \mu \mathrm{m})}{\mathrm{erg} \mathrm{s}^{-1}}\right) .
$$

We realize that there are several alternative tracers for

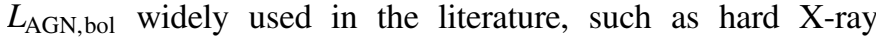
luminosity, mid-IR AGN continuum luminosity, and the midIR [O IV] $\lambda 25.89 \mu \mathrm{m}$ emission line luminosity (Meléndez et al. 2008; Diamond-Stanic et al. 2009; Rigby et al. 2009).

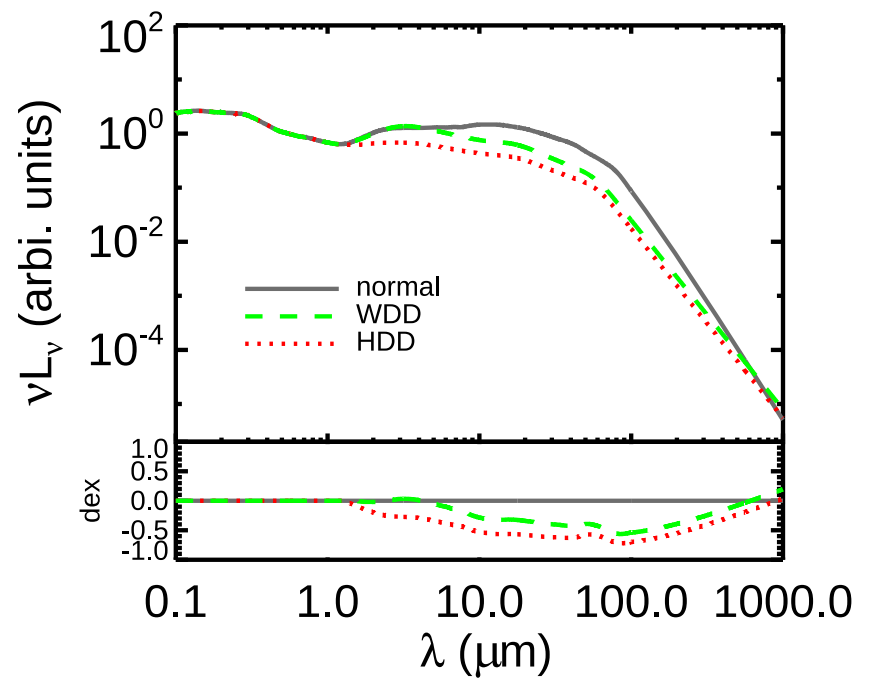

Figure 8. Comparison of the normal, WDD, and HDD AGN templates developed in Lyu et al. (2017). The bottom panel highlights the IR flux differences in dex of these templates by normalizing their SEDs by the normal AGN template.

Although these tracers could be less affected by dust obscuration and might be isotropic, whether they can be applied over a wide AGN luminosity range that covers the quasar population is a question. For example, the application of the [O IV] emission line has only been discussed for the Seyfert population $\left(L_{\mathrm{AGN}, \text { bol }} \lesssim 10^{11} L_{\odot}\right)$, and there is evidence suggesting the structures of AGN narrow-line regions could evolve at high luminosity (e.g., Netzer et al. 2004). Using the single-band mid-IR continuum is also complicated: (1) the fraction of the AGN luminosity that is reprocessed in the mid-IR $(7-15 \mu \mathrm{m})$ can vary intrinsically by $0.3-0.9$ dex (Lyu et al. 2017), and (2) the possible addition of reprocessed emission from AGNheated polar dust can easily lead to scatter exceeding 1 dex for the same $L_{\mathrm{AGN}, \text { bol }}$ (Lyu \& Rieke 2018). Given these uncertainties, we opt to use the $V$-band bolometric correction throughout this work.

The ambiguities in calculating AGN bolometric luminosity have contributions from both measured qualities (e.g., the photometric errors) and bolometric correction factors. In many cases, the latter can dominate. Given the likely variation from source to source, we adopt $0.3 \mathrm{dex}$ as the relative $L_{\mathrm{AGN}}$ uncertainty for all objects.

\subsubsection{Correlation between Mid-IR Time-Lag and AGN Luminosity}

In Figure 9, we present the time-lags of the mid-IR emission in the WISE W1 and W2 bands of PG quasars as a function of their AGN bolometric luminosities. It is clear that these two quantities are strongly correlated. From fitting these measurements with Equation (10) with the IDL program FITEXY, we have

$$
\Delta t_{\text {torus, } W 1} / \text { day }=10^{2.10 \pm 0.06}\left(L_{\mathrm{AGN}, \mathrm{SED}} / 10^{11} L_{\odot}\right)^{0.47 \pm 0.06}
$$

for the $W l$ band and

$$
\Delta t_{\text {torus }, W 2} / \text { day }=10^{2.20 \pm 0.06}\left(L_{\mathrm{AGN}, \mathrm{SED}} / 10^{11} L_{\odot}\right)^{0.45 \pm 0.05}
$$

for the $W 2$ band. These correlations closely follow the expected $\Delta t \propto L_{\mathrm{AGN}}^{0.5}$ relation. At a given $L_{\mathrm{AGN}}$, the time-lag differences in the $W 1$ and $W 2$ bands are small, with a mean value of $\Delta t_{\text {torus, } W 2} / \Delta t_{\text {torus, } W 1} \sim 1.21 \pm 0.36$ and a median value at 

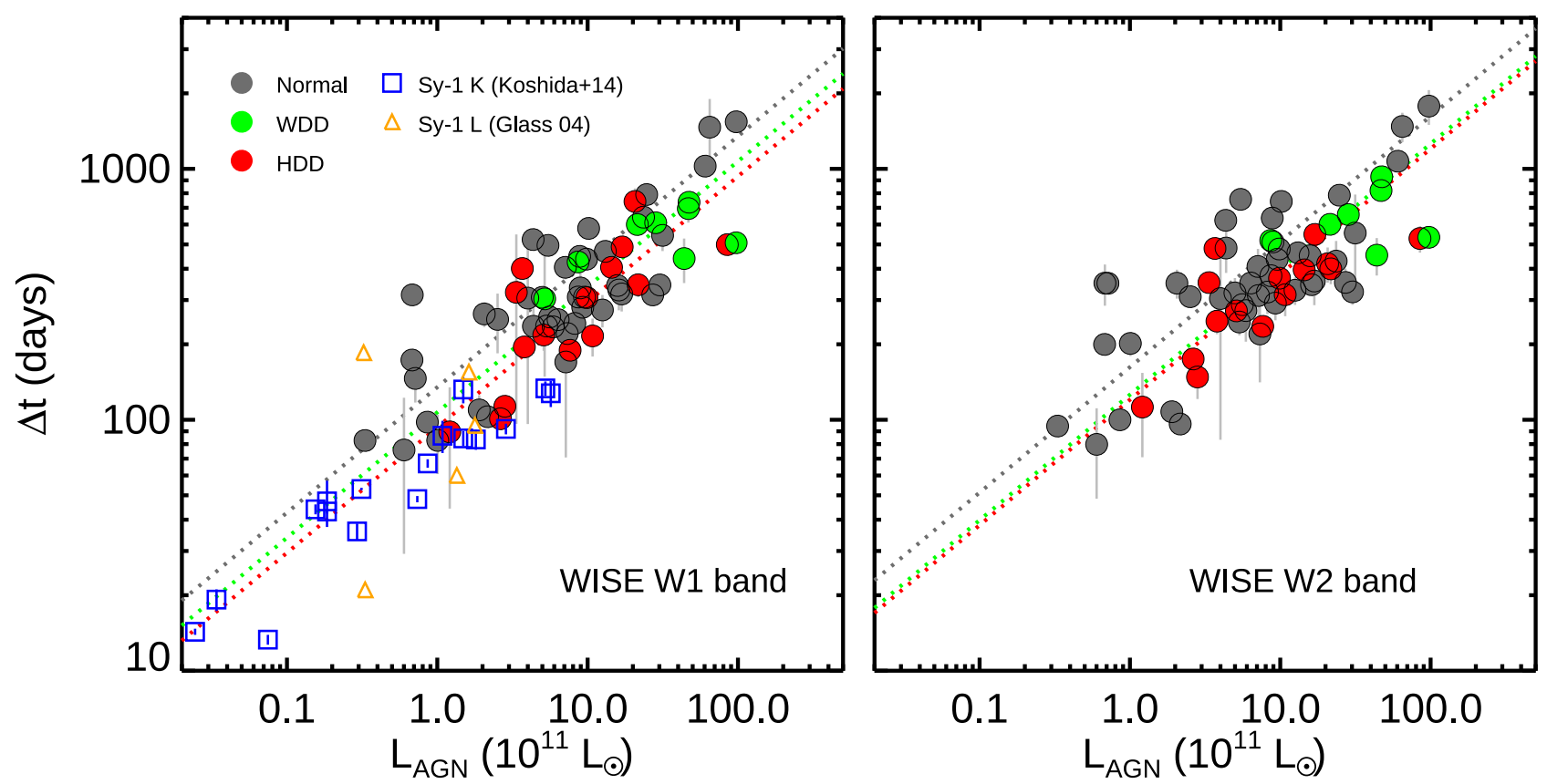

Figure 9. Dust time-lags between the WISE mid-IR band and optical band light curves plotted against the AGN luminosity for PG quasars. The dotted lines are the fitted correlations for normal (gray), WDD (green), and HDD (red) quasars assuming $\Delta t \propto L_{\mathrm{AGN}}^{0.5}$. We also show the $K$-band time-lag measurements of the 17 Seyfert 1 nuclei studied in Koshida et al. (2014) and the $L$-band time-lag measurements of the five Seyfert 1 nuclei in Glass (2004), with our estimation of their AGN bolometric luminosities in the left panel.

\subsection{A linear fit to both $W 1$ and $W 2$ time-lags yields}

$$
\Delta t_{\text {torus, } W 2}=(1.17 \pm 0.11) \Delta t_{\text {torus }, W 1}+(0.21 \pm 54.3) .
$$

Despite the large uncertainty, the fitted intercept is very close to zero, indicating that the time-lag in $W 2$ is always statistically larger than that in $W I$ by a constant factor.

The average redshift of the 67 fitted PG quasars is $0.15 \pm 0.10$. Assuming a normal AGN template, the corresponding intrinsic wavelengths of the WISE WI- and W2-band filters are $2.94_{-0.24}^{+0.28}$ and $4.03_{-0.33}^{+0.38} \mu \mathrm{m}$. For an AGN with $L_{\mathrm{AGN}, \text { bol }}=10^{12} L_{\odot}$, these results correspond to a dust emission region size at $\lambda_{\text {rest }} \sim 3 \mu \mathrm{m}$ of $\sim 0.31 \mathrm{pc}$ for the $W 1$ band and at $\lambda_{\text {rest }} \sim 4 \mu \mathrm{m}$ of $\sim 0.37 \mathrm{pc}$ for the $W 2$ band. The similar sizes at the $W 1$ and $W 2$ bands strongly support a compact torus, where the emission at the rest frame $\sim 3-5 \mu \mathrm{m}$ is dominated by dust grains with similar temperatures.

\subsubsection{Comparison with Previous Dust Reverberation Studies}

Previous ground-based $K$-band RM has only been focused on relatively low-luminosity AGNs $\left(L_{\mathrm{AGN}} \lesssim 10^{11} L_{\odot}\right)$ in Seyfert galaxies. However, given the SED analysis in Lyu \& Rieke (2018), it is likely that Seyfert 1 nuclei and quasars share similar torus properties. For the 17 Seyfert 1 objects in Koshida et al. (2014), we adopted the average $K$-band time-lags estimated with the CCF methods and the $\alpha_{\nu}=1 / 3$ accretion disk component model and converted the weighted averaged

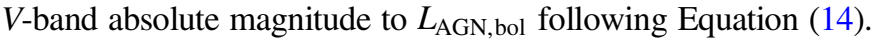
(See Appendix C for further discussion of the consistency of their and our measurements of time-lags and AGN luminosities.) Applying the same correlation analysis to the Seyfert $1 \mathrm{~K}$-band time-lag and AGN bolometric luminosity yields

$$
\Delta t_{\text {torus }, K} / \text { day }=10^{1.86 \pm 0.06}\left(L_{\mathrm{AGN}, \mathrm{SED}} / 10^{11} L_{\odot}\right)^{0.45 \pm 0.07} .
$$

For $L_{\mathrm{AGN} \text {,bol }}=10^{12} L_{\odot}$, the corresponding $K$-band torus size $\left(\lambda_{\text {rest }} \sim 2.1 \mu \mathrm{m}\right)$ is $0.17 \mathrm{pc}$, which is about half the WISE WI size. Such a difference could provide important insights into the properties of the AGN innermost torus, which will be discussed in detail in another paper. Both the $K$-band and $W 1$ behavior are consistent with a general scaling as $L^{0.5}$ over a range of 10,000 in luminosity, demonstrating that similar circumnuclear torus structures are common to AGNs in general.

Glass (2004) provided preliminary time-lag measurements between the $U(\sim 0.36 \mu \mathrm{m})$ and $L(\sim 3.4 \mu \mathrm{m})$ bands of five Seyfert 1 nuclei: Fairall 9, Akn 120, NGC 3783, ESO 141G55, and NGC 7469. We have analyzed the IR SEDs of all of these AGNs with our empirical templates in Lyu \& Rieke (2018). Following Section 4.2.1, we estimate their $L_{\mathrm{AGN}}$ and plot their locations in Figure 9. These objects have a very limited luminosity range $\left(L_{\mathrm{AGN}}=10^{10.5} \sim 10^{11.2} L_{\odot}\right)$, so it is not possible to pin down a meaningful lag-luminosity relation given the uncertain time-lag measurements and small sample. Nevertheless, they distribute around the prediction of our timelag correlations based on the WISE W1- and W2-band data of PG quasars.

The optically reddened type-1 AGN NGC 6418 is the only object with published robust mid-IR dust reverberation results. With high-cadence Spitzer/IRAC monitoring, Vazquez et al. (2015) found that this object had time-lags of 37.2 days at $3.6 \mu \mathrm{m}$ and 47 days at $4.5 \mu \mathrm{m}$. Based on the $\mathrm{H} \alpha$ luminosity of NGC 6418, they determined a lower limit to the AGN bolometric luminosity, $L_{\mathrm{AGN}, \text { bol }} \geqslant 5 \times 10^{9} L_{\odot}$. With our previously developed AGN templates (Lyu et al. 2017; Lyu \& Rieke 2017), we can estimate the upper limit on its AGN bolometric luminosity to be $\lesssim 6.3-20 \times 10^{9} L_{\odot}$ (depending on whether the AGN is normal, WDD, or HDD) based on its WISE W4 flux and SED shape. If NGC 6418 has similar torus 
Table 5

PG Quasar Variability at 12 and $22 \mu \mathrm{m}$

\begin{tabular}{|c|c|c|c|c|c|c|c|c|c|c|c|}
\hline Name & MJD & $\begin{array}{c}f_{12 \mu \mathrm{m}} \\
\mathrm{mJy}\end{array}$ & $\begin{array}{l}\text { Error } \\
\text { mJy }\end{array}$ & $\begin{array}{c}\text { Stdev } \\
\text { of change }\end{array}$ & $\Delta M_{W 3}$ & MJD & $\begin{array}{c}f_{22 \mu \mathrm{m}} \\
\mathrm{mJy}\end{array}$ & $\begin{array}{l}\text { Error } \\
\text { mJy }\end{array}$ & $\begin{array}{c}\text { Stdev } \\
\text { of change }\end{array}$ & $\Delta M_{W 4}$ & Comment \\
\hline \multirow[t]{2}{*}{ PG $0003+158$} & $53,721.5$ & 13.62 & 0.88 & & & $53,721.5$ & 24.41 & 2.06 & & & \\
\hline & $55,376.1$ & 13.96 & 0.34 & -0.36 & 0.03 & $55,376.1$ & 25.02 & 1.24 & -0.25 & 0.03 & \\
\hline \multirow[t]{2}{*}{ PG $0003+199$} & $53,559.2$ & 175.20 & 3.62 & & & $\cdots$ & $\cdots$ & $\cdots$ & & & \\
\hline & $55,377.8$ & 191.29 & 2.53 & -3.65 & 0.10 & $55,377.8$ & 280.42 & 5.60 & & & \\
\hline \multirow[t]{2}{*}{ PG $0007+106$} & $53,721.3$ & 74.01 & 2.75 & & & $53,721.3$ & 153.34 & 3.90 & & & \\
\hline & $55,375.0$ & 64.94 & 1.00 & 3.10 & 0.15 & $55,375.0$ & 126.65 & 2.79 & 5.56 & 0.23 & Variable, FSRQ \\
\hline \multirow[t]{2}{*}{ PG $0026+129$} & $53,592.9$ & 30.56 & 1.50 & & & $53,725.8$ & 45.84 & 2.34 & & & \\
\hline & $55,379.5$ & 32.70 & 0.96 & -1.20 & 0.08 & $55,379.5$ & 43.63 & 3.55 & 0.52 & 0.05 & \\
\hline \multirow[t]{2}{*}{ PG $0043+039$} & $53,381.3$ & 14.28 & 1.55 & & & $53,381.3$ & 22.14 & 2.03 & & & \\
\hline & $55,383.0$ & 16.03 & 0.55 & -1.06 & 0.13 & $55,382.0$ & 25.22 & 2.12 & -1.05 & 0.15 & \\
\hline \multirow{2}{*}{ PG $0049+171$} & $53,750.4$ & 13.60 & 0.99 & & & $53,750.4$ & 19.02 & 2.40 & & & \\
\hline & $55,342.6$ & 15.76 & 0.34 & -2.07 & 0.17 & $55,42.6$ & 17.96 & 1.17 & 0.40 & 0.06 & \\
\hline$\cdots$ & $\ldots$ & $\ldots$ & $\ldots$ & $\ldots$ & $\ldots$ & $\ldots$ & $\ldots$ & $\ldots$ & $\cdots$ & $\cdots$ & $\cdots$ \\
\hline
\end{tabular}

(This table is available in its entirety in machine-readable form.)

properties as PG quasars, given the similar bandpasses of WISE and Spitzer/IRAC, its AGN bolometric luminosity should be about $8-9 \times 10^{9} L_{\odot}$ based on Equations (15) and (16) for the reported time-lags. This value is well above the lower limit constrained by Vazquez et al. (2015) and below the upper limit given by its mid-IR emission. As a result, we conclude that there is no evidence for strong differences in the torus structures between this low-luminosity AGN and quasars in the mid-IR.

\section{3. $P G$ Quasar Variability at $10-25 \mu m$}

Among the 87 PG quasars at $z \lesssim 0.5,33$ have repeated MIPS measurements at $24 \mu \mathrm{m}$. In Table 2, we present the flux change significance, $S_{i, j}$, between (1) the first two MIPS measurements, (2) the third measurement (if acquired) and the previous measurement that shows the greatest difference, and (3) the average of the MIPS measurements and the WISE measurement, if the latter is available. The significance of a change in flux is given by

$$
S_{i, j}=\frac{\left|f_{i}-f_{j}\right|}{\sqrt{\sigma_{i}^{2}+\sigma_{j}^{2}}},
$$

where $f_{i}, f_{j}$ are the two flux measurements and $\sigma_{i}, \sigma_{j}$ are the corresponding uncertainties. We identify variability when one of these values satisfies $S_{i, j} \geqslant 3$ (similar to the $3 \sigma$ criterion given its definition). Among 26 radio-quiet $\mathrm{PG}$ objects, only one, PG $1535+547$, was found to vary at $24 \mu \mathrm{m}$. For the remaining seven radio-loud quasars, two out of three flatspectrum $(\alpha>-0.7)^{9}$ but none of four steep-spectrum $(\alpha<-0.7)$ objects were found to vary. It appears that AGN $24 \mu \mathrm{m}$ variability is related to the radio-band classification. In the upcoming section, we will expand the statistics with a larger sample of 139 quasars with similar observations.

We also explore the 12 and $22 \mu \mathrm{m}$ variability of the $87 \mathrm{PG}$ quasars by comparing Spitzer/IRS synthetic photometry to the WISE measurements (see Table 5). With a $3 \sigma$ threshold, only nine objects ( $10 \%$ of the sample) have $12 \mu \mathrm{m}$ flux

\footnotetext{
9 We assume a radio spectrum with $f_{\nu} \propto \nu$, where $f_{\nu}$ is the observed flux density and $\nu$ is the observed frequency.
}

variations, and two objects ( $2 \%$ of the sample) have $22 \mu \mathrm{m}$ flux variations. Because there may be systematic errors of a few percent between data obtained from different observatories (Carey 2010; Sloan et al. 2015), in addition to the statistical uncertainties, a $5 \sigma$ significance level is more convincing for variability detection. With this requirement, only two quasars, PG 1226+023 (3C 273) and PG 0007+106 (a flat-spectrum radio source and blazar at $z=0.089$; Mao et al. 2016), are left. This is not surprising, since previous studies of blazars and flatspectrum radio sources have found them to vary substantially at $10 \mu \mathrm{m}$ (e.g., Rieke 1972; Rieke \& Kinman 1974; Edelson \& Malkan 1987; Neugebauer \& Matthews 1999). In particular, 3C 273 is a well-known IR-variable object (e.g., Neugebauer \& Matthews 1999; Soldi et al. 2008). It seems that most, if not all, significant AGN IR variability at $\lambda \gtrsim 10 \mu \mathrm{m}$ can be associated with nonthermal processes.

\subsection{AGN Variability at $22-24 \mu m$ in a Larger Sample}

\subsubsection{General Variability Behavior}

With the multi-epoch MIPS and WISE measurements of 139 objects, we now further probe AGN variability at $22-24 \mu \mathrm{m}$ with a much larger sample. The measurements and results are also listed in Table 2 and shown graphically in Figure 10 . We use $q_{24}=\log \left[f_{\nu}(24 \mu \mathrm{m}) / f_{\nu}(1.4 \mathrm{GHz})\right]$ to determine radioloudness and tag as radio-loud (RL) for $q_{24}<-0.5$, radiointermediate (RI), for $-0.5<q_{24}<0.5$, and radio-quiet (RQ) for $q_{24}>0.5$. The radio data were taken from the NASA Extragalactic Database (NED), the FIRST survey (Kellermann et al. 1989, 2016; both corrected to $1.4 \mathrm{GHz}$ assuming a slope of -0.7), Hodge et al. (2011), and the Molonglo Galactic Plane Survey (Murphy et al. 2007). There is one anomalous case, PG $1309+355$, which has a flat radio spectrum but is radiointermediate by our definition; by other criteria, however, it is radio-loud (Laor et al. 2019). We have counted it among the radio-loud flat-spectrum radio quasars (FSRQs). Sources lacking radio data are by default included with the radio-quiet sample. Because there was no difference in variability behavior, we have also combined the radio-intermediate sources with this sample. Hereafter, this mixed sample is referred as "radio-quiet" for brevity. 


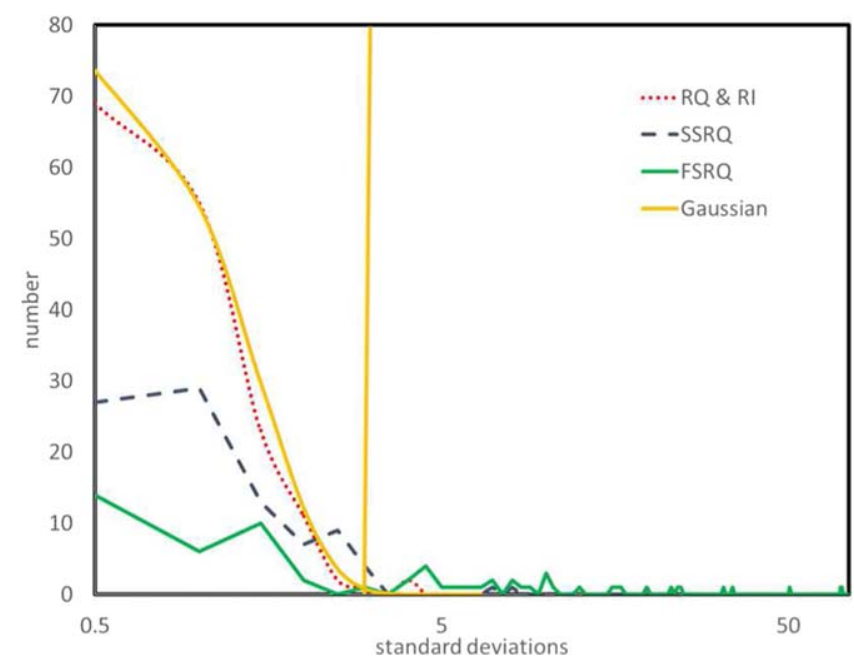

Figure 10. Distribution of variability significance $S_{i, j}$ between different $24 \mu \mathrm{m}$ measurements in all 139 quasars. The calculated $S_{i, j}$ have been linearly binned from zero with a bin size of 0.5. A Gaussian with $\sigma=0.9$ has been binned similarly. The vertical orange line indicates $S_{i, j}=3$ used to identify variability. Two objects, 2MASSi J0918486+211717 and PG 1535+547, stand out as having larger changes and appear to be exceptional radio-quiet variables. Although only one steep-spectrum quasar is identified as being probably variable, their distribution of variability significances is broader than that for the radio-quiet sources, suggestive of low-level variability in some of them. Compared with steep-spectrum radio quasars, the distribution for the FSRQs is even broader and indicates a strong link between this radio characteristic and variability at $24 \mu \mathrm{m}$.

As shown in Figure 10, the flux change significance distribution of radio-quiet AGNs is best fitted with a Gaussian of width of 0.9 - that is, this fit indicates that the estimates of individual standard deviations are high (conservative) by about $10 \%$ under the assumption that the sample is not variable. ${ }^{10}$ This result confirms that our criterion for identifying variations is conservative and furthermore emphasizes how rare $24 \mu \mathrm{m}$ variability is for these objects. As indicated by the $10 \%$ smaller width of the Gaussian fit to the radio-quiet population, the real systematic error might be smaller than $0.7 \%$, as introduced in Section 2.1.3. However, we decided not to change it, since a uniform correction would not apply across the entire sample (e.g., faint sources probably have larger errors). Given these uncertainties, two sources, the FSRQ NGC 1275 and the steepspectrum radio quasar (SSRQ) 3C 270, are identified as possibly being variable because they are detected at very high $\mathrm{S} / \mathrm{N}$ and show flux changes at $2.7 \sigma-2.8 \sigma$. Overall, we detect $24 \mu \mathrm{m}$ variations in 22 (including the two probable variables) out of 139 targets and set tight constraints on possible variations for the rest of the sample within the limits of our observational cadence.

The steep-spectrum radio-loud sources show a distribution of the $24 \mu \mathrm{m}$ flux change significance that is somewhat broader than that for the radio-quiet sample, suggesting that low levels of IR variability may apply for some of them, even if it is not detected individually. However, their overall pattern is not very different from that of the radio-quiet sample. In contrast, the distribution of flat-spectrum sources is very broad, showing that variability is common among them. In fact, we detect variations in 14 out of 19 FSRQs - a very high fraction given our small number of observations.

\footnotetext{
${ }^{10}$ Since PG $1309+355$ has not varied in our observations, classifying it as radio-intermediate would have no effect on this result.
}

\subsubsection{Other Variability Studies}

It seems possible that the measurements by IRAS at $25 \mu \mathrm{m}$ could extend the timescale of our survey. However, the IRAS photometry is of relatively low accuracy. There are variations at the $5 \%$ level (up to $10 \%$ ) in the response along a pixel, and the simple bulk photoconductors used are susceptible to responsivity shifts due to exposure to ionizing radiation (Beichman et al. 1988). Evidence for a significant $(>3 \sigma)$ flux change after applying these systematic errors is found for only three objects: 3C 273, 3C 274, and PKS 2005-189. The increased brightness of 3C 274 as seen by IRAS can be explained by its resolved nature, evident in the Spitzer images. Shi et al. (2007) showed that the extended $24 \mu \mathrm{m}$ emission is the IR synchrotron radiation from the outer jet and inner radio lobes of this nearby FR I-type radio galaxy. All of the extended emission would be included within the IRAS beam, and photometry of the Spitzer images using a $1^{\prime}$ aperture completely recovers the excess flux measured by IRAS. However, the IRAS data do demonstrate that 3C 273 was nearly twice as bright and PKS 2005-189 about three times as bright when measured in 1983 compared with our measurements. Both of these sources are FSRQ blazars, so these results help document the extent of their variability but do not qualitatively change the findings above.

Neugebauer \& Matthews (1999) presented a multiyear photometry monitoring for $25 \mathrm{PG}$ quasars from 1 through $10 \mu \mathrm{m}$ ( $J$ through $N$ band) and reported $10 \mu \mathrm{m}$ variability in $3 \mathrm{C}$ 273. They also deduced possible variations at $10 \mu \mathrm{m}$ for PG $1535+547$ on the basis that its mid-IR light curve tended to follow the variations seen at shorter wavelengths, even if by themselves the measurements did not have enough $\mathrm{S} / \mathrm{N}$ to make a persuasive detection. They also used a statistical argument to make a case for variations in other radio-loud objects.

\subsubsection{Lack of $24 \mu \mathrm{m}$ Variations in Radio-quiet Quasars}

Our study therefore represents a substantial advance over previous work on the variability of AGNs at 10 and $20 \mu \mathrm{m}$. We now use it to evaluate the overall variability of the radio-quiet sample by computing the weighted average of the absolute changes in flux between the initial two MIPS measurements. ${ }^{11}$ Although we have only two measurements per object, the large sample size lets us put interesting limits on variability using a statistical approach.

The repeatability of the MIPS $24 \mu \mathrm{m}$ photometry in the absence of any photon noise can be as good as $0.4 \%$ (Engelbracht et al. 2007). If we remove 2MASSi J0918486 +211717 and PG 1535+547, plus all of the blazars and flatspectrum radio sources, the rms scatter in our quasar measurements is $0.0085 \pm 0.0006 \mathrm{mag}(0.79 \% \pm 0.06 \%)$; if we further remove all radio-loud objects, the rms scatter reduces to $0.0076 \pm 0.0006 \mathrm{mag}(0.70 \% \pm 0.06 \%)$. That is, outside of 2MASSi J0918486+211717 and PG 1535+547, plus the flat-spectrum radio sources and blazars, the rms scatter in the measurements is consistent with virtually no variability over the $\sim 3$ yr period spanned by the MIPS observations.

\footnotetext{
${ }^{11}$ We focus on the initial two MIPS $24 \mu \mathrm{m}$ measurements instead of the WISE results, since they are generally of higher $\mathrm{S} / \mathrm{N}$ and use a smaller beam that reduces contamination issues. Also, the first two epochs of observations encompassed the entire sample, whereas the third MIPS observational epoch only includes radio-loud AGNs.
} 

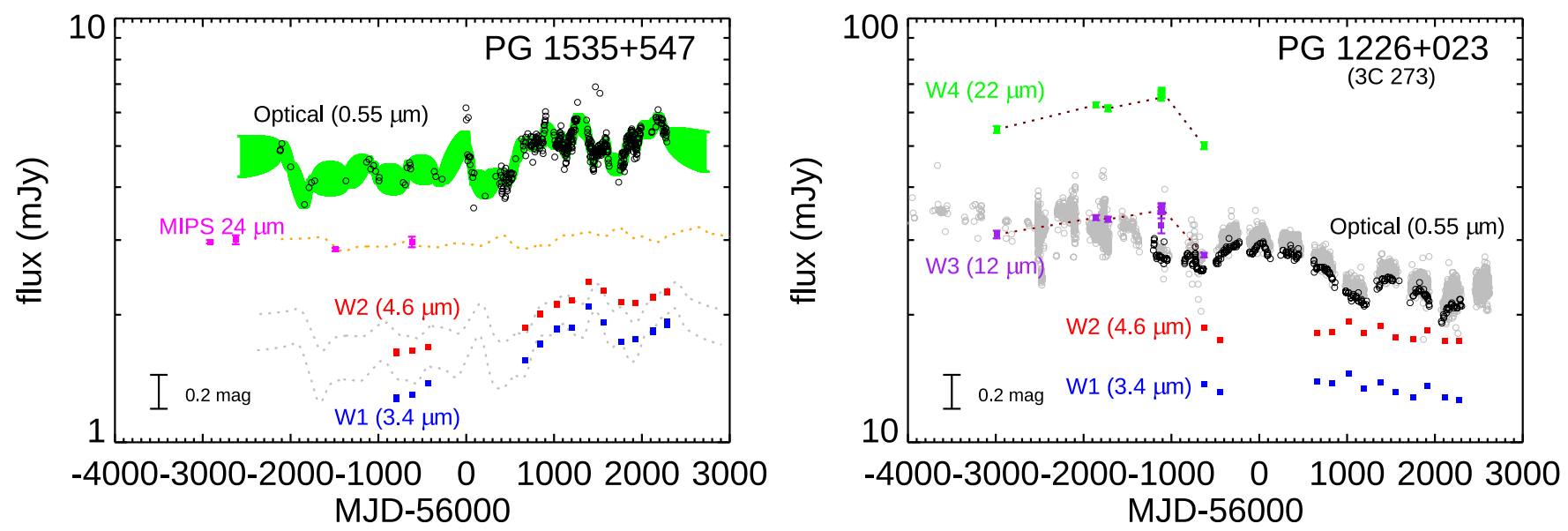

Figure 11. Multiband IR and optical light curves of the radio-quiet PG $1535+547$ (left) and the blazar PG $1226+023$ (right). See the text for details.

Now we compare these $24 \mu \mathrm{m}$ upper limits to the variability in the WISE $W 1$ and $W 2$ bands, i.e., at 3-5 $\mu \mathrm{m}$. For the entire PG sample, the rms scatter is 0.086 and 0.071 mag at $W 1$ and $\mathrm{W} 2$, respectively. For the subset for which we have $24 \mu \mathrm{m}$ measurements, the values are the same. That is, variability at $3.4 \mu \mathrm{m}$ is a factor of 10 larger than the upper limits set for $24 \mu \mathrm{m}$. As deduced by Neugebauer \& Matthews (1999), the variability damps out dramatically going from the near- to midIR. The Spitzer measurements put this result on much sounder ground than previously: to illustrate the gain, at $73 \mathrm{mJy}, \mathrm{PG}$ $1535+547$ is close to the median brightness of the sample, 86 $\mathrm{mJy}$, yet its variations were just at the detection limit with the previous ground-based data.

\subsubsection{Behavior of Variable Radio-quiet Quasars}

The objects PG 1535+547 and 2MASSi J0918486+211717 are of interest as apparently normal, radio-quiet, optically selected quasars that have exceptional mid-IR variability signals at 10 and $24 \mu \mathrm{m}$; PG $1535+547$ (Mrk 486) is of particular interest because it is very thoroughly studied. Lyu et al. (2017) found its IR SED to be fitted very well by their WDD template, which makes the assumption that the nuclear nonthermal continuum has dropped to a negligible level by $24 \mu \mathrm{m}$ and that the mid-IR flux comes mostly from the circumnuclear torus with very little contribution from polar dust. Xie et al. (2017) showed it to have weak silicate emission features, requiring that some, if not all, of its $24 \mu \mathrm{m}$ emission is from heated dust. In the optical band, PG $1535+547$ is moderately obscured with a red continuum, and it has the strongest polarization signals reported in the PG sample ( $p \sim 2.5 \%$; Berriman et al. 1990). Indeed, Hubble Space Telescope UV and ground-based spectropolarimetry shows that the polarization rises to nearly $8 \%$ in the UV and that there are complex polarization changes across the $\mathrm{H} \alpha$ and $\mathrm{H} \beta$ emission line profiles (Smith et al. 1997). The presence of strong emission lines in the polarized flux spectrum and the strong rise of the polarization into the UV suggests that dust scattering of the AGN nuclear continuum and emission from the BLR is the polarizing mechanism. Despite the fact that the polarization is from scattering, Smith et al. (1997) found evidence that the optical polarization varies on timescales as short as a year. In total, these observations indicate that PG $1535+547$ cannot be face-on but must be partially obscured by its circumnuclear torus. In this case, even if a jet is present, its apparent emission would not be strongly amplified by relativistic beaming; hence, it would be weak due to its misalignment with our line of sight, and it would not contribute significantly to the observed flux at 10 and $24 \mu \mathrm{m}$ (see more discussion in Section 5.1). Instead, we suggest that the IR variations of PG $1535+547$ at longer wavelengths are dust reverberation signals.

To illustrate the different mid-IR variable behaviors of radioquiet and radio-loud quasars, we compare the IR light curves of PG 1535+547 and 3C 273 in Figure 11. For the blazar 3C 273, there is no obvious similarity between its optical and mid-IR light curves, especially given the large variations seen between the neighboring epochs in WISE W2. The brightness of 3C 273 at $W 3$ and $W 4$ varied by $\sim 0.3$ mag, about 0.15 mag larger than the variations observed in $W 1$ and W2. Such behavior is not expected from the dust emission, since its energy output at longer wavelengths must correspond to larger physical scales, where the amplitude of variability is expected to decrease.

In contrast, the W1- and W2-band light curves of PG 1535 +547 can be reproduced by shifting and scaling its optical light curve, as expected for dust reverberation signals with time-lags of $\sim 180$ days for $W 1$ and $\sim 205$ days for W2. Assuming a timelag 2.5 times larger (i.e., $\sim 450$ days) than that of $W l$ and an amplitude factor of 0.6 , the $24 \mu \mathrm{m}$ light curve can be matched by dust RM. The variability amplitude of PG $1535+547$ in W1 is $\gtrsim 0.5 \mathrm{mag}$, among the $10 \%$ largest $W 1$-band variables in the PG sample. In comparison, the $24 \mu \mathrm{m}$ variability amplitude of PG $1535+547$ is about 10 times smaller at only $\sim 0.06$ mag. The observed behavior of this object suggests that a detectable level of mid-IR variability caused by dust reverberation is plausible given a highly variable quasar, high-S/N data, and fortunate time sampling.

\section{Discussion}

\subsection{The Origin of AGN Mid-IR Variability}

\subsubsection{Synchrotron Emission}

The Spitzer $24 \mu \mathrm{m}$ results reaffirm the generally accepted picture that the smooth UV-millimeter continua of blazars is dominated by the variable, optically thin synchrotron radiation produced in the core and inner relativistic jet that also gives rise to the self-absorbed, flat-spectrum radio emission at centimeter wavelengths (see e.g., Kellermann \& Pauliny-Toth 1981). According to the classical unification scheme (Urry \& Padovani 1995), this type of object corresponds to an AGN 


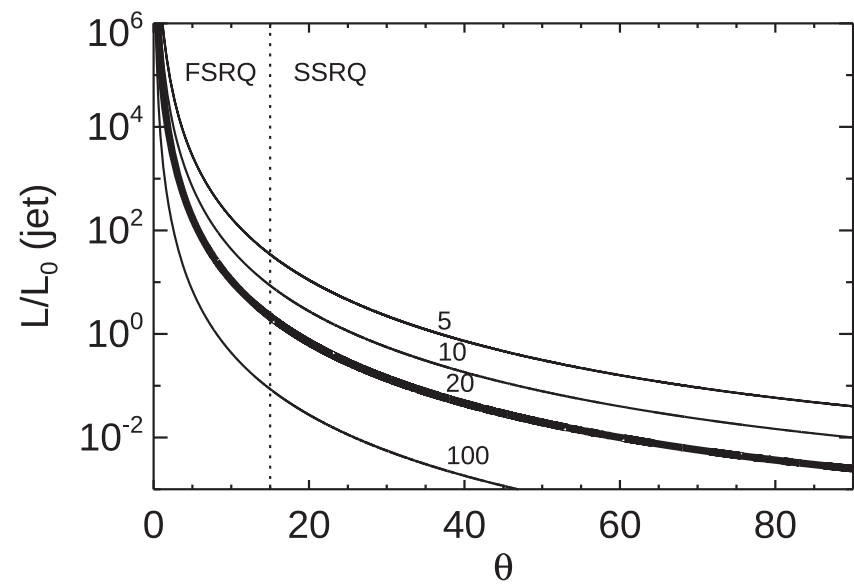

Figure 12. Flux boosting factor as a function of observing angle for different Lorentz factors $(\gamma=5,10,20,100)$.

with a powerful jet pointed toward the observer, and its broadband X-ray-to-IR SED is dominated by synchrotron emission that is enhanced by relativistic beaming.

As shown in Section 4.3, among the radio-loud AGNs, SSRQs seem to lack strong variability at $24 \mu \mathrm{m}$, in contrast to FSRQs. As suggested by Urry \& Padovani (1995), FSRQs are believed to be oriented at relatively small angles to the line of sight $\left(\theta \lesssim 15^{\circ}\right)$, whereas SSRQs have intermediate angles between FSRQ and FR II radio galaxies. For an ideal relativistic beam, the apparent luminosity can be calculated as $L=L_{0} /[\gamma(1-\beta \cos \theta)]^{2}$, where $\gamma$ is the Lorentz factor, $L_{0}$ is the intrinsic luminosity, and $\beta=\left(1-\gamma^{-2}\right)^{1 / 2}$ is the beam speed in the AGN frame in units of light speed $c$ (Cohen et al. 2007). For blazars, Lorenz factors of up to 10-20 are common for parsec-scale jets (Homan 2012). Assuming $\gamma=20$, the jet variability signal can be boosted by a factor of 2-1600 for FSRQs (see Figure 12), while the apparent variability for SSRQs decreases quickly to a few percent or smaller when $\theta>15^{\circ}$. In other words, given the same level of intrinsic jet variability, FSRQs are expected to have much higher (synchrotron) IR variability detection rates compared with SSRQs. In addition, it is possible that the IR emission of the SSRQs is no longer dominated by the relativistic jet but by the emission of a circumnuclear torus. In that case, their behavior would resemble that of radio-quiet quasars, which do not vary significantly within our $24 \mu \mathrm{m}$ observations.

Given the arguments above, the different mid-IR variability behavior among SSRQs, FSRQs, and blazars can be ascribed to first order to different angles of the radio jets relative to the observer. This result is consistent with the unification picture for radio-loud AGNs (Urry \& Padovani 1995).

\subsubsection{Dust IR Reverberation Signals}

The mid-IR variations of the radio-quiet quasars show little evidence for any synchrotron component but are dominated by the dust response to the UV-optical variations of the central engine. As summarized in Section 4.1, the 3-5 $\mu \mathrm{m}$ IR emission of $\sim 77 \%$ of the PG quasars at $z<0.5$ shows such dust reverberation signals. If we set aside the 16 sources where the data were inadequate to look for time-lags, $95 \%$ of the remaining objects behave in this way, with only four objects (5\%) showing an alternative pattern of variations. This is strong evidence for the common existence of circumnuclear dust outside the $\mathrm{BH}$ accretion disk.
The near-IR SEDs of quasars with normal or WDD behavior (Lyu \& Rieke 2017) show direct evidence for this circumnuclear dust heated by the accretion disk in the form of a spectral bump at $\sim 3 \mu \mathrm{m}$ that matches the expected emission of dust heated to sublimation temperatures. Although HDD quasars do not show a similar near-IR bump, as shown in Figure 9, they still follow the $R \propto L^{1 / 2}$ size-luminosity relation, and their dust time-lags increase with wavelength. This is true even for the most HDD quasar, PG 0049+171, where we detect time-lags $\sim 120$ days for its $W 1$ variations and $\sim 157$ days for $W 2$. This behavior is evidence that the emission near $3 \mu \mathrm{m}$ is dominated by the emission of the circumnuclear torus and not by the accretion disk/central engine. Furthermore, since the IR SEDs of unobscured quasars at $z \sim 0-6$ have similar forms as the PG sample (Lyu et al. 2017), the result implies that there are few, if any, completely dust-free quasars.

For radio-quiet quasars, the average rms variability amplitudes at the $V, W 1$, and $W 2$ bands are $0.145,0.093$, and 0.076 mag. In other words, the $W 1$ and $W 2$ dust variation signals are typically only $\sim 60 \%$ and $\sim 50 \%$ of those in the optical band. As presented in Section 4.3, the possible flux change at $24 \mu \mathrm{m}$ is less than $10 \%$ of that at $\sim 3 \mu \mathrm{m}$ (corresponding to $6 \%$ of the variability at the $V$ band). The decrease in variability with increasing wavelength is likely to be the result of averaging over the variations due to light travel time to various parts of the extended circumnuclear torus. Diminished variability at $24 \mu \mathrm{m}$ then results from the emission originating in a substantially larger region, i.e., at a significantly larger radius than the emission at 3-4 $\mu \mathrm{m}$.

\subsubsection{AGN Mid-IR Variability under the Unification Scheme}

Our RM study has focused on the PG quasars because their selection criteria strongly favor cases where we can see the central engine, accretion disk, and circumnuclear torus all relatively unobscured. We discuss here the complexities that can be expected where this simple situation is not the case.

In general, both synchrotron emission and torus-reprocessed emission will contribute to the mid-IR variability of a radioloud AGN; their relative importance depends on the inclination angle and radio-loudness. In addition, the obscuration of the central engine will substantially affect the apparent optical variability, making the interpretation of the mid-IR light curves difficult. Figure 13 provides an illustration of the classical unification model (Antonucci 1993; Urry \& Padovani 1995) and shows the optical and mid-IR continuum light curves of various AGN components.

In the radio-loud case, the integrated optical continuum emission of the AGN is a combination of the jet component $\left(f_{\text {jet,opt }}(t)\right)$ and the accretion disk $\left(f_{\mathrm{AD}, \text { opt }}(t)\right)$,

$$
F_{\text {radio-loud,opt }} \approx C_{\text {torus }}(\theta) f_{\mathrm{AD}, \mathrm{opt}}(t)+C_{\text {beam }}(\theta) f_{\text {jet }, \mathrm{opt}}(t),
$$

where $C_{\text {torus }}(\theta)$ reflects the obscuration level of the accretion disk light by the torus $\left(C_{\text {torus }} \sim 1\right.$ for type- 1 AGNs and $\sim 0$ for type-2 AGNs) and $C_{\text {beam }}(\theta)$ is the flux boosting factor due to realistic beaming. As the presumed inclination angle of the jet to our line of sight increases from BL Lac to FSRQ to SSRQ, the relative contribution of $f_{\mathrm{AD} \text {, opt }}$ increases quickly. For narrow-line radio galaxies (NLRGs), although the absolute strength of the jet variability is weaker than that for the SSRQ case, the optical light curve is dominated by the jet, since the 


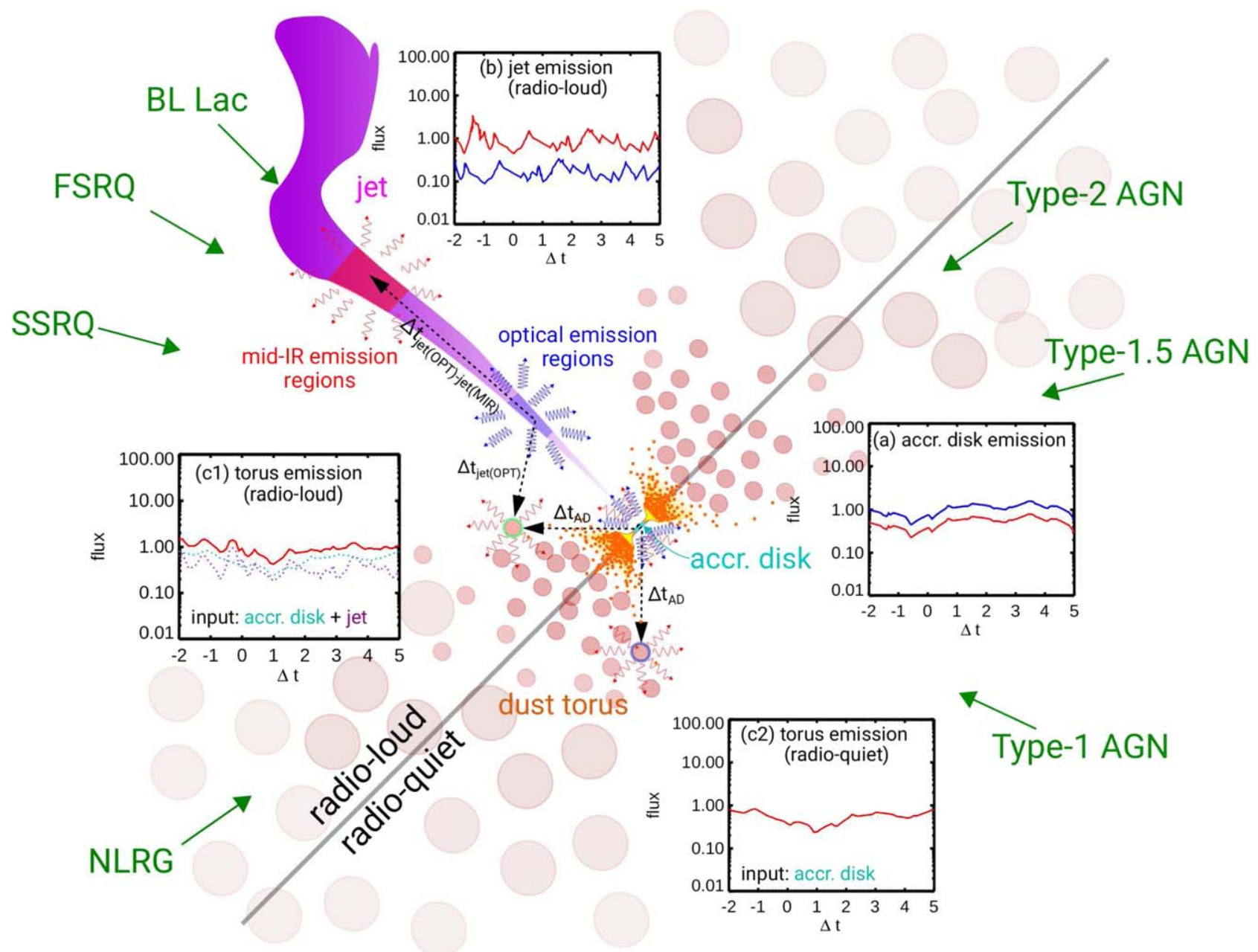

Figure 13. Sketch of the main AGN structures related to the AGN optical and mid-IR continuum variabilities. At the very center is the BH and its accretion disk (cyan). The dust torus is assumed to be clumpy, and yellow, orange, and red represent a decreasing dust temperature. A strong jet (purple) exists for the radio-loud case, and we highlight its optical emission (blue) and mid-IR emission (red) regions. For the accretion disk and jet component, we plot their optical (blue solid line) and mid-IR (red solid line) light curves in panels (a) and (b). The mid-IR light curves (red solid line) of the torus in the radio-loud and radio-quiet cases are plotted in panels (c1) and (c2). For the radio-loud case, we show the contribution from the dust reverberation signals to the accretion disk and the jet in dashed cyan and purple lines. According to the AGN unification model (Antonucci 1993; Urry \& Padovani 1995), we denote different AGN types at different inclination angles for radio-loud and radio-quiet cases with green arrows. See the text for detailed explanations.

accretion disk is obscured $\left(C_{\text {torus }} \sim 0\right)$. In principle, both the jet component and the accretion disk can provide UV-optical energy to heat the torus and produce the mid-IR emission. As they locate in different regions, the mid-IR dust reverberation signals of the torus might be mixed with the two separated time-lags $\left(\Delta t_{\text {jet(opt) }}\right.$ and $\left.\Delta t_{\mathrm{AD}}\right)$. The relativistic beaming effects also apply to the jet IR emission, so the observed integrated mid-IR emission can be written as

$$
\begin{aligned}
& F_{\text {radio-loud,IR }} \approx f_{\mathrm{AD}, \mathrm{IR}}(t)+C_{\text {beam }}(\theta) f_{\text {jet,IR }}(t) \\
& \quad+\mathcal{F}_{\mathrm{AD}, \text { opt }}\left(t-\Delta t_{\mathrm{AD}}\right)+\mathcal{F}_{\text {jet,opt }}\left(t-\Delta t_{\text {jet(opt })}\right),
\end{aligned}
$$

where we use $\mathcal{F}(t-\Delta t)$ to denote the dust-reprocessed emission of the optical light $f(t)$ with a time-lag $\Delta t$. Since the jet is highly beamed and perpendicularly distributed, its illumination to the torus could often be ignored. As a result, the observed total IR emission can be further simplified into

$$
\begin{aligned}
F_{\text {radio-loud,IR }} \approx & f_{\mathrm{AD}, \text { IR }}(t)+C_{\text {beam }}(\theta) f_{\text {jet, IR }}(t) \\
& +\mathcal{F}_{\mathrm{AD}, \text { opt }}\left(t-\Delta t_{\mathrm{AD}}\right) .
\end{aligned}
$$

Comparing it with the form of $F_{\text {radio-loud,opt }}$, if dominated by different components, the optical and mid-IR light curves could be uncorrelated (the jet component and accretion disk have different variability patterns). This could explain the behavior of 3C 273 (as seen in Figure 11).

The situation for radio-quiet AGNs is much simpler; their optical light curve is $F_{\text {radio-quiet,opt }} \approx C_{\text {torus }}(\theta) f_{\mathrm{AD} \text {,opt }}(t)$, and their mid-IR light curve is $F_{\text {radio-quiet,IR }} \approx f_{\mathrm{AD}, \mathrm{IR}}(t)+$ $\mathcal{F}_{\mathrm{AD}, \text { opt }}\left(t-\Delta t_{\mathrm{AD}}\right)$. When the nucleus is unobscured $\left(C_{\text {torus }} \sim\right.$ 1 ), we readily observe the time-lag $\Delta t_{\mathrm{AD}}$, which reflects the light travel time from the accretion disk to the torus, as demonstrated by our dust RM. However, if the nucleus is obscured, the optical band could have a significant contribution from the host galaxy. Given the large beams used for much of our visible data, in this situation, it would be difficult to extract the nuclear component accurately. To illustrate this issue, consider the full sample observed at $24 \mu \mathrm{m}$, excluding the radio-loud sources. Of the remaining sources, $50 \%$ are 2 MASS quasars, which show varying amounts of AGN obscuration (e.g., Cutri et al. 2002). Of the remaining sources, $40 \%$ are of 
Table 6

Results of Linear Regression of Lag-Luminosity Relation

\begin{tabular}{|c|c|c|c|c|c|c|c|}
\hline \multirow{2}{*}{$\begin{array}{l}\text { Sample } \\
\text { (1) }\end{array}$} & \multirow{2}{*}{$\begin{array}{c}\text { Observed Band } \\
\text { (2) }\end{array}$} & \multirow{2}{*}{$\begin{array}{l}<z> \\
(3)\end{array}$} & \multirow{2}{*}{$\begin{array}{c}\lambda_{\text {rest }} \\
(\mu \mathrm{m}) \\
(4)\end{array}$} & \multirow{2}{*}{$\begin{array}{c}\text { Sample Size } \\
\text { (5) }\end{array}$} & \multicolumn{2}{|c|}{$\alpha$} & \multirow{2}{*}{$\begin{array}{l}\beta \\
(8)\end{array}$} \\
\hline & & & & & $\left(\Delta L_{\mathrm{AGN}}=0.3 \mathrm{dex}\right)$ & $\begin{array}{c}\left(\Delta L_{\mathrm{AGN}}=0.025 \mathrm{dex}\right) \\
(7)\end{array}$ & \\
\hline \multirow[t]{2}{*}{ Normal QSOs } & $W 1$ & 0.145 & 2.95 & 44 & $2.13 \pm 0.04$ & $2.21 \pm 0.01$ & 0.5 \\
\hline & $W 2$ & 0.145 & 4.04 & 44 & $2.21 \pm 0.04$ & $2.28 \pm 0.01$ & 0.5 \\
\hline \multirow[t]{2}{*}{ WDD QSOs } & $W 1$ & 0.220 & 2.78 & 8 & $2.03 \pm 0.09$ & $2.04 \pm 0.05$ & 0.5 \\
\hline & $W 2$ & 0.241 & 3.72 & 7 & $2.10 \pm 0.09$ & $2.12 \pm 0.05$ & 0.5 \\
\hline \multirow[t]{2}{*}{ HDD QSOs } & $W 1$ & 0.122 & 3.01 & 15 & $1.97 \pm 0.07$ & $1.98 \pm 0.04$ & 0.5 \\
\hline & $W 2$ & 0.122 & 4.12 & 15 & $2.08 \pm 0.07$ & $2.08 \pm 0.04$ & 0.5 \\
\hline \multirow[t]{2}{*}{ All QSOs } & $W 1$ & 0.149 & 2.94 & 67 & $2.08 \pm 0.03$ & $2.19 \pm 0.01$ & 0.5 \\
\hline & $W 2$ & 0.149 & 4.03 & 66 & $2.16 \pm 0.03$ & $2.27 \pm 0.01$ & 0.5 \\
\hline Seyfert 1 & $K$ & 0.038 & 2.11 & 17 & $1.88 \pm 0.05$ & $1.93 \pm 0.02$ & 0.5 \\
\hline
\end{tabular}

The fitted model is $\log (\Delta t)=\alpha+\beta \log \left(L_{\mathrm{AGN}}\right)$.

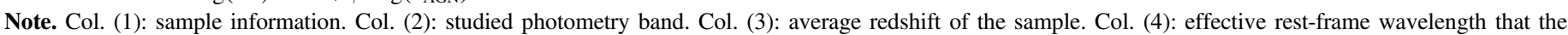

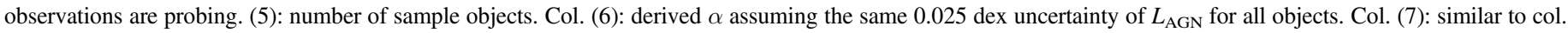
(5), but the uncertainty is assumed to be 0.3 dex. Col. (8): $\beta$ is fixed to 0.5 .

types $1.8,1.9$, or 2 (from the NED database), again indicating substantial obscuration.

\subsubsection{Other Causes of IR Variations}

Besides the radio-loud PG $1226+023$ (3C 273), four other PG objects-PG 0003+199, PG 0923+201, PG 1534+580, and PG 1612+261-have uncorrelated optical and mid-IR variability. None of these objects are blazars or even radioloud, so it is unlikely that nonthermal emission contributes to their IR variations. In addition, since all of these objects show blue optical continua and strong broad Balmer emission lines, the optical light curve should delineate the variations of the accretion disk. Consequently, their IR variations cannot be the dust reverberation signals from a classical torus. We cannot rule out the possibility that these objects have very complicated dust structures (e.g., there are several polar dust clouds or filaments) that cause reverberation signals with a wide range of time-lags with diverse luminosity weights-for some configurations, the integrated IR light curve might not show any variation pattern similar to the input optical signals. Nevertheless, there is no new mechanism involved in this possibility -it still belongs to the dust reverberation signals.

Dynamical motion of the torus material could be another possibility to cause AGN IR variability. Assuming the dusty environment of an AGN can be described as a radiation-driven fountain, Schartmann et al. (2014) explored the time-resolved IR SEDs and argued for possible variations, but over a timescale of $0.1 \mathrm{Myr}$. As their simulation indicates, there could be significant but temporary dust in the line of sight that is heated by the AGN, causing changes in the IR (by dust emission) and optical flux (by dust extinction). On yearly timescales, however, the turbulence/fountain argument cannot explain the observed mid-IR variations of these four quasars by merely moving dust clouds. Taking the typical velocities of order $300-400 \mathrm{~km} \mathrm{~s}^{-1}$, the moving dusty clouds/filaments could only travel a few $10^{-4} \mathrm{pc}$, which would not change the exposed solid angle significantly at a distance of a parsec from the heating source.

However, there is a chance that dust grains could be evaporated when the dusty clouds/filaments move to an environment with strong radiation heating by the AGN or where an outburst (which could be at a nonvisible wavelength, e.g., X-rays) by the central engine evaporates dust in a formerly "safe" environment. As a result, their IR emission could be quickly reduced. In fact, the dust destruction timescale around the sublimation region is estimated to be on the order of 10 days (Kishimoto et al. 2013), quick enough to produce the midIR variation we see. Observed optical polarization variability seen in objects like PG0050+124, PG1535+547 (Smith et al. 1997), and Mrk 231 (Gallagher et al. 2005) on timescales of less than a year may also be related to rapid sublimation events. In another paper, we will provide a detailed study of how to explain these peculiar mid-IR variability features with this possibility and discuss its broad implications.

In conclusion, (1) observed mid-IR variability on monthly to yearly timescales of most quasars is caused by dust reverberation signals that are correlated with the variations of the accretion disk emission. The amplitude of the dust IR variability decreases quickly with increasing wavelength. (2) If a jet is present and the system is viewed close to the line of sight to the jet axis (e.g., FSRQ or blazar), nonthermal processes dominate the IR variations with larger variation amplitudes over shorter timescales. (3) Only a small subset of quasars appears to have IR variability that does not fall into these two categories, which might be explained by reverberation signals off structures other than the circumnuclear torus or by the motions of dusty clumps combined with the heating and destruction of the constituent dust grains.

\subsection{Constraints on the Torus Properties}

\subsubsection{Wavelength-dependent Torus Size and Issues with the Classical Clumpy Model}

To compare the torus sizes constrained through dust reverberation signals at different wavelengths, we fix the slope of the time-lag-versus-AGN luminosity relation to be 0.5 and repeat the fitting for $W 1$ - and $W 2$-band measurements of the PG quasars. The results can be found in Table 6 . For the 67 objects with convincing dust time-lags, we find that the dust emission size ratios follow $R_{W 1}: R_{W 2}=1: 1.2$. As suggested by Lyu et al. (2017), quasars having different intrinsic IR SED variations may be a reflection of different torus structures. Therefore, we also separately compute the corresponding $\Delta t-L_{\mathrm{AGN}}$ relations for normal, WDD, and HDD populations. Discussion of how the torus size depends on SED type will be 
expanded in Section 5.2.3. Here we simply assume that all AGNs have a similar torus structure.

We also make a similar correlation analysis for the $K$-band reverberation signals of the 17 Seyfert 1 sample in Koshida et al. (2014), finding a size ratio between the $K$ and $W l$ bands of $R_{K}: R_{W 1}=0.6: 1$. In Appendix C.1, we present the SED decomposition results of these Seyfert 1 nuclei and estimate that half of the sample should have intrinsic IR SEDs best described by the normal AGN template. This value is similar to the 60\%-70\% normal AGN fraction in the PG quasar sample (Lyu et al. 2017). As a result, the AGN dust emission size differences at the $K$ and $W 1$ bands are unlikely to be related to the different AGN IR SED types. To test whether the results depend strongly on the uncertainties in the AGN luminosity estimate, we changed the uncertainty of $L_{\mathrm{AGN}}$ from 0.3 to $0.025 \mathrm{dex}$ (the latter value corresponds to the typical $V$-band magnitude uncertainty of Seyfert 1 nuclei in Koshida et al. 2014) and repeated the fits. The $\Delta t-L_{\mathrm{AGN}}$ correlations shift by $0.05-0.1 \mathrm{dex}$, but the size ratios between $K, W 1$, and $W 2$ are not significantly affected, i.e., $R_{K}: R_{W 1}: R_{W 2}=0.6: 1: 1.2$.

The poor time sampling of the $24 \mu \mathrm{m}$ observations prevents us from deriving any torus size through RM. On the other hand, the lack of variability at this wavelength is most readily explained if the torus zone dominating the $24 \mu \mathrm{m}$ emission is much larger than the zone producing the bulk of the flux observed at $W 1$ and $W 2$. In this case, the time-lags have a large range so that the variability is smoothed out.

These observational results provide some challenges to the predictions based on the classical "clumpy" torus picture. Almeyda et al. (2017) simulated the IR reverberation response of such a torus model and suggested that the dust time-lags at 3-5 $\mu \mathrm{m}$ have very limited wavelength dependence (see their Section 4.1). In addition, their model also predicted substantial reverberation signals at 10 and $30 \mu \mathrm{m}$ (see their Figure 5). These conclusions are expected with their basic model assumption: from the illuminated face to the dark side, the same optically thick clouds can have a broad temperature distribution that results in IR emission over a wide wavelength range. However, this picture is not favored by our observations. Given the typical redshift of the sources we have observed at $24 \mu \mathrm{m}$, the rest wavelength is $\sim 20 \mu \mathrm{m}$. Interpolating their results at 10 and $30 \mu \mathrm{m}$ for an extended clumpy torus, the same model would predict a peak response at the wavelength of our observations of about $35 \%$ of the response at rest 3.6 and $4.5 \mu \mathrm{m}$. In contrast, we have found that the response at $24 \mu \mathrm{m}$ is an order of magnitude smaller than that at (observed) 3.4 and $4.5 \mu \mathrm{m}$.

Rather than being purely clumpy, the torus is likely to be a mixture of optically thick dusty clouds and some diffuse distribution of low-density dust. Such a structure is likely because clumps at the inner edge of the torus are not likely to be completely stable against gravitational shearing and hence must be sustained as a result of turbulence or similar effects. In fact, it is known that the clumpy torus model (e.g., Nenkova et al. 2008) alone cannot reasonably fit the $\sim 3 \mu \mathrm{m}$ hot dust emission peak among quasars, and another blackbody with $T \sim 1300 \mathrm{~K}$ needs to be added (e.g., Mor et al. 2009; Leipski et al. 2013). In contrast, the two-phase (clumps + diffuse dust) torus model developed by Stalevski et al. $(2012,2016)$ does not have this problem and appears to reproduce the AGN hot dust emission features.
Previous work has often argued that a single blackbody plus a power-law SED is enough to reproduce the quasar continuum at $\lambda \sim 0.5-3 \mu \mathrm{m}$ (e.g., Glikman et al. 2006; Kim et al. 2015; Hernán-Caballero et al. 2016). Since dust temperature is a strong function of distance, the dust grains responsible for the $\lambda \sim 1-3 \mu \mathrm{m}$ emission should be located at similar radii. However, our study finds contradictory evidence with $R_{K}: R_{W 1}=0.6: 1$ and indicates a complicated picture. Interestingly, under the assumption of similar grain sizes, the sublimation radii for graphite and silicate dust grains have $R_{\text {sub, } \mathrm{C}} / R_{\text {sub }, \mathrm{S}} \sim 0.5$ (see Section 4.2 ), very close to the reported $R_{K}: R_{W 1} \sim 0.6$. Future work should address whether this possibility can be supported by other evidence, as well as folding in the constraints on torus structure provided by the relative lack of variability at $24 \mu \mathrm{m}$.

\subsubsection{Surface Density Profiles of the Hot Dust Emission}

Previously, the radial structures of AGN tori have been explored at $8-13 \mu \mathrm{m}$ only by long-baseline IR interferometry observations of $<10$ Seyfert nuclei (Kishimoto et al. 2009, 2011). Assuming a power-law radial surface density distribution of heated dust, the profile ranges from $\sim r^{0}$ to $\sim r^{-1}$ and might be dependent on AGN luminosity (Kishimoto et al. 2011). However, it is likely that most of these objects have some contribution of extended polar dust emission in the midIR $\left(f_{\text {pol }, 10 \mu \mathrm{m}} / f_{\text {total }, 10 \mu \mathrm{m}} \sim 0.3-0.8\right.$ estimated from SED analysis in Lyu \& Rieke 2018), so the real density profile of the compact torus at these wavelengths is still highly uncertain.

With the measurements of variability amplitude differences between the optical and IR light curves, crude constraints on the dust surface density profile can be calculated with some simple approximations.

For simplicity, we assume the dust sublimation zone is smooth (i.e., not clumpy) and that its surface density profile can be described by a power law $\Sigma(r) \propto r^{\alpha}$. In addition, the dust grains in the sublimation zone will have only a modest range of temperature, given that the AGN hot dust emission feature is well matched by a single blackbody spectrum (e.g., Mor et al. 2009). Furthermore, the dust grains will be hot enough that the temperature-dependence of their emission will be modest (i.e., they approach the Rayleigh-Jeans regime). Therefore, we can ignore the radial dependence of the temperature and express the IR emission as

$$
f_{\mathrm{IR}} \propto \int \Sigma(r) r d r \propto r^{\alpha+2}
$$

Since the hot dust time-lag has been found to be linearly correlated with the square root of AGN luminosity, we can argue that the dust distance $r$ is correlated to the AGN optical luminosity by the inverse square law, $r \propto L_{\mathrm{opt}}^{0.5}$, so

$$
f_{\mathrm{IR}} \propto f_{\mathrm{opt}}^{(\alpha+2) / 2} \text {. }
$$

When the optical flux $f_{\text {opt }}$ is changed to $f_{\text {opt }}^{\prime}$ by a factor of $\xi$, the corresponding IR flux changes from $f_{\mathrm{IR}}$ to $f_{\mathrm{IR}}^{\prime}$, and

$$
\frac{f_{\mathrm{IR}}^{\prime}}{f_{\mathrm{IR}}}=\left(\frac{f_{\mathrm{opt}}^{\prime}}{f_{\mathrm{opt}}}\right)^{(\alpha+2) / 2}=\xi^{(\alpha+2) / 2} .
$$


Table 7

Parameters Related to the Torus Radial Density Profiles

\begin{tabular}{lccc}
\hline \hline Parameter & $K$ & $W 1$ & $W 2$ \\
\hline$\left\langle\lambda_{\text {rest }}\right\rangle / \mu \mathrm{m}$ & 2.1 & 3.0 & 4.1 \\
$\Delta M_{\mathrm{IR}}$ & $0.48 \pm 0.33$ & $0.19 \pm 0.11$ & $0.16 \pm 0.09$ \\
$\mathrm{AMP}$ & $4.65 \pm 3.07$ & $4.65 \pm 3.51$ & $4.68 \pm 3.47$ \\
$f_{\mathrm{IR}} / f_{\text {opt }}$ & 4.16 & 6.90 & 9.49 \\
$\alpha$ & 0.3 & -0.7 & -1 \\
\hline
\end{tabular}

With our RM model, the absolute flux change in the IR is correlated with that in the optical according to Equation (4),

$$
\Delta f_{\mathrm{IR}}=f_{\mathrm{IR}}^{\prime}-f_{\mathrm{IR}}=\mathrm{AMP} \times(\xi-1) f_{\mathrm{opt}} .
$$

We can rewrite Equation (25) as

$$
1+\operatorname{AMP}(\xi-1) \frac{f_{\mathrm{opt}}}{f_{\mathrm{IR}}}=\xi^{(\alpha+2) / 2}
$$

The IR variability can be characterized by magnitude change $\Delta M_{\mathrm{IR}}$

$$
\Delta M_{\mathrm{IR}}=-2.5 \log \left(\frac{f_{\mathrm{IR}}^{\prime}}{f_{\mathrm{IR}}}\right) .
$$

Finally, we have

$$
\alpha=2\left(\frac{\log \left(10^{-\Delta M_{\mathrm{IR}} / 2.5}\right)}{\log \left(1+\left(10^{-\Delta M_{\mathrm{IR}} / 2.5}-1\right)\left(f_{\mathrm{IR}} / f_{\mathrm{opt}}\right) \mathrm{AMP}^{-1}\right)}-1\right),
$$

where $f_{\mathrm{IR}} / f_{\text {opt }}$ is the IR-to-optical color of the AGN SED.

We summarize the mean values of $\Delta M$ and AMP of the Seyfert 1 AGN and PG quasar samples in Table 7. Assuming a normal AGN template, the characteristic dust surface density profiles are found to be $r^{0.3}$ in $K, r^{-0.7}$ in $W 1$, and $r^{-1.0}$ in $W 2$. Such a result suggests that the surface density gradient of sublimating dust increases gradually with radius at $2.0 \mu \mathrm{m}$, reaches a peak after that, and drops quickly at longer wavelengths.

\subsubsection{Normal Quasars versus Dust-deficient Quasars}

Lyu et al. (2017) showed that the intrinsic IR SEDs of PG quasars can be grouped into normal, WDD, and HDD populations that are likely associated with different torus structures. Now we discuss whether such arguments are supported by the dust reverberation results. Considering the relatively small numbers of dust-deficient quasars in our PG sample, we reduce the number of free parameters by assuming that the time-lag goes exactly as the square root of the luminosity (i.e., $\beta=0.5$ ) to facilitate comparisons of the timelag-AGN luminosity relations for the different quasar types. The fitted parameters can be found in Table 6. At the same AGN luminosity, WDD and HDD quasars appear to have smaller time-lags (see also Figure 9), suggesting a more compact hot dust emission zone.

In Figure 14, we compare the variation amplitudes and timelags for different populations of quasars. First, there is no strong correlation between these two properties. The HDD quasars have relatively smaller variation amplitudes compared with the normal quasar population, as expected from their

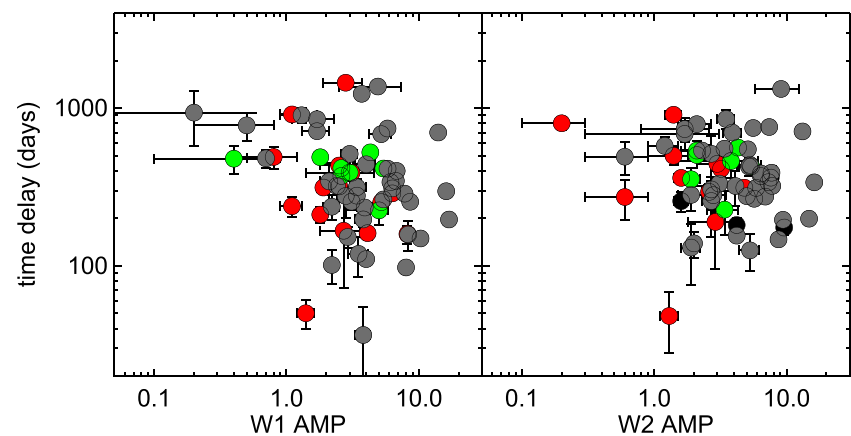

Figure 14. Distribution of variation amplitudes and time-lags for normal (gray), WDD (green), and HDD (red) quasars.

Table 8

K-S Probabilities of the HDD and WDD Quasars against Normal Quasars

\begin{tabular}{lccc}
\hline \hline Measurement & HDD & WDD & HDD+WDD \\
\hline W1 AMP & $\mathbf{0 . 0 0 9}^{\mathrm{a}}$ & 0.315 & 0.014 \\
W1 lag & 0.947 & 0.046 & 0.439 \\
W2 AMP & $\mathbf{0 . 0 0 5}$ & 0.227 & $\mathbf{0 . 0 0 5}$ \\
W2 lag & 0.949 & 0.351 & 0.847 \\
\hline
\end{tabular}

Note.

${ }^{\mathrm{a}}$ We indicate significant differences $(p<0.01)$ in bold.

different SED features. However, the WDD quasars do not show much of a difference. This is possibly related to their similar SEDs to normal quasars at these wavelengths.

We have carried out Kolmogorov-Smirnov (K-S) tests to check whether the distributions of the variation amplitudes and time-lags among dust-deficient quasars and normal quasars are different at a significant level. The K-S probabilities that describe the likelihood that the two samples do not differ significantly can be found in Table 8. As demonstrated in Lyu et al. (2017), the distribution of AGN luminosities among HDD quasars is not significantly different from that for normal quasars. For the mid-IR time-lags, the K-S probabilities of the HDD against normal quasars are close to unity $(p \sim 0.95)$. In other words, the time-lag distributions of the $W 1$ and $W 2$ bands between the HDD population and the normal population are similar (but their dependences on AGN luminosity are different, as shown in Table 6). However, the variation amplitude distribution between HDD and normal quasars is significantly different $(p \sim 0.009$ for $W 1$ and $p \sim 0.005$ for W2), which can be expected if HDD quasars have fewer hot dust grains heated by the AGN, as previously proposed based on SED features in Lyu et al. (2017). For WDD quasars, although the K-S test results do not support any significant differences with normal quasars, the situation is uncertain, since WDD quasars have higher redshifts and luminosities. These two features would impact the observed torus size in the opposite direction, hindering meaningful comparisons.

\subsection{Relation between the Dusty Torus and BLRs}

In the classical AGN unification scheme, the BLR is dustfree, relatively small, and well separated from the outer dusty torus (Antonucci 1993; Urry \& Padovani 1995). In recent years, proposals have been made that the BLR is a failed dusty wind from the outer accretion disk (Czerny \& Hryniewicz 2011). Under this picture, Baskin \& Laor (2018) explored the expected dust properties and corresponding BLR structure and 
Table 9

Time-lag Comparison of BLR and Mid-IR Dust for 12 PG Quasars

\begin{tabular}{lccccc}
\hline \hline Name & Type & $\log \left(L_{\mathrm{AGN}, \mathrm{bol}} / L_{\odot}\right)$ & $\Delta t_{\mathrm{H} \alpha}$ & $\Delta t_{\mathrm{H} \beta}$ & $\Delta t_{W 1}$ \\
\hline PG 0026+129 & HDD & 12.44 & $132_{-31}^{+29}$ & $125_{-31}^{+29}$ & $577.0 \pm 20.4$ \\
PG 0052+251 & HDD & 12.56 & $211_{-44}^{+66}$ & $99_{-31}^{+30}$ & $360.0 \pm 75.1$ \\
PG 0804+761 & WDD & 12.55 & $193_{-17}^{+20}$ & $151_{-24}^{+26}$ & $659.9 \pm 40.4$ \\
PG 8444+349 & HDD? & 11.87 & $39_{-16}^{+16}$ & $13_{-11}^{+14}$ & $224.0 \pm 26.4$ \\
PG 0953+414 & WDD & 12.93 & & $187_{-33}^{+27}$ & $913.0 \pm 68.7$ \\
PG 1211+143 & Norm & 12.13 & $116_{-46}^{+38}$ & $103_{-44}^{+32}$ & $365.6 \pm 22.4$ \\
PG 1229+204 & Norm & 11.53 & $71_{-46}^{+39}$ & $36_{-18}^{+32}$ & $266.3 \pm 2.3$ \\
PG 1307+085 & HDD & 12.16 & $179_{-144}^{+94}$ & $108_{-115}^{+46}$ & $357.6 \pm 25.2$ \\
PG 1411+442 & Norm & 12.02 & $103_{-37}^{+40}$ & $118_{-71}^{+72}$ & $441.7 \pm 26.2$ \\
PG 1426+015 & Norm & 12.09 & $90_{-68}^{+46}$ & $115_{-68}^{+49}$ & $264.0 \pm 34.3$ \\
PG 1613+658 & Norm & 12.42 & $43_{-22}^{+40}$ & $44_{-23}^{+20}$ & $371.9 \pm 86.3$ \\
PG 2130+099 & Norm & 11.90 & $237_{-28}^{+53}$ & $\cdots$ & 0.3 \\
\end{tabular}

argued for the presence of large graphite grains $(a \gtrsim 0.3 \mu \mathrm{m})$ down to the observed size of the BLR. By comparing the RM analysis of the BLR and dusty torus sizes, these statements can be tested.

Previous comparisons of $K$-band and UV-optical emission line time-lags relative to the optical continuum have suggested that the torus inner radius is larger than the BLR region, with $R_{\mathrm{BLR}} \sim 0.5 R_{\mathrm{TOR}, K}$ for Seyfert 1 nuclei (Suganuma et al. 2006; Koshida et al. 2014). This result was obtained by comparing the time-lag-AGN luminosity correlation for the BLR and torus from different samples. A very small number of objects have size estimates of both the torus (in the near-IR band) and the BLR from dust and emission line RMs at similar observing epochs. Clavel et al. (1989) reported $R_{\mathrm{BLR}, \mathrm{MgII}} / R_{\mathrm{TOR}, K} \sim 0.4$ for Fairall 9, a bright quasar with $L_{\mathrm{AGN} \text {,bol }} \sim 3 \times 10^{12} L_{\odot}$. Pozo Nuñez et al. (2015) found $R_{\mathrm{BLR}, \mathrm{H} \alpha} / R_{\mathrm{TOR}, K} \sim 0.4$ for PGC 50424, a Seyfert 1 nucleus with $L_{\mathrm{AGN}, \text { bol }} \sim 3 \times 10^{10} L_{\odot}$. Ramolla et al. (2018) studied the optical and near-IR time-lags of 3C 120, a type- $1 \mathrm{AGN}$ with $L_{\mathrm{AGN}}$,bol $\sim 4 \times 10^{11} L_{\odot}$, and argued for $R_{\mathrm{BLR}, \mathrm{H} \alpha} / R_{\mathrm{TOR}, K} \sim 0.7$.

With our mid-IR reverberation analysis and previous BLR size measurements of the PG sample, we can explore the BLR and torus relation in a large sample of bright quasars. Kaspi et al. (2000) studied the variations in the optical Balmer emission lines and the continuum emission of 28 PG quasars and reported time-lag measurements for 17 objects. Among these 17 objects, we have detected mid-IR time-lags in 15 . The results are summarized in Table 9. The mean value of $\Delta t_{\mathrm{BLR}} / \Delta t_{\mathrm{TOR}, 3.0 \mu \mathrm{m}} \sim 0.23 \pm 0.10$, with a maximum value of 0.44 , minimum value of 0.06 , and median of 0.23 .

In Figure 15, we explore whether there are correlations between $\Delta t_{\mathrm{BLR}}$ and $\Delta t_{\mathrm{TOR}, \mathrm{W} 1}$. Linear fits result in

$$
\Delta t_{\mathrm{BLR}, \mathrm{H} \alpha}=(0.37 \pm 0.06) \Delta t_{\mathrm{TOR}, W 1}+(-43.00 \pm 26.49)
$$

and

$$
\Delta t_{\mathrm{BLR}, \mathrm{H} \beta}=(0.28 \pm 0.05) \Delta t_{\mathrm{TOR}, W 1}+(-44.18 \pm 21.98) .
$$

In $\log -\log$ space, we find

$$
\Delta t_{\mathrm{BLR}, \mathrm{H} \alpha}=10^{-2.64 \pm 0.29}\left(\Delta t_{\mathrm{TOR}, W 1}\right)^{1.77 \pm 0.11}
$$

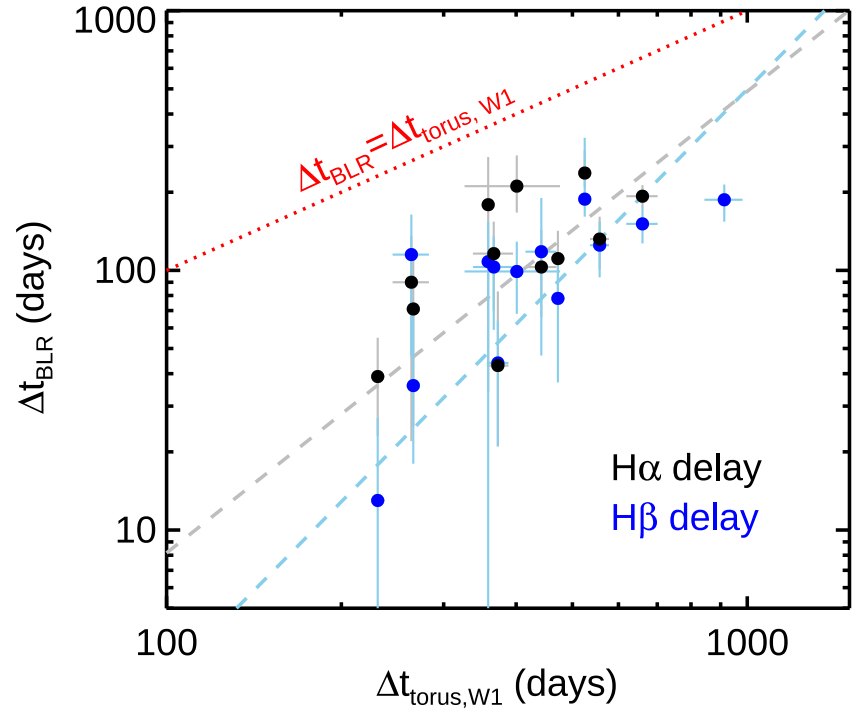

Figure 15. Comparison of the IR light lags in the WISE WI band $(\sim 3.4 \mu \mathrm{m})$ from this work and the broad emission line lags of 17 PG quasars from Kaspi et al. (2000). We fit linear functions for $\mathrm{H} \beta$ lag vs. $W 1$ lag (blue dots) and $\mathrm{H} \alpha$ lag vs. W1 lag (black dots) separately, shown as blue and black dashed lines (see the text for details). The red dotted line represents where the torus IR time lags equal the BLR time lags.

and

$$
\Delta t_{\mathrm{BLR}, \mathrm{H} \beta}=10^{-4.12 \pm 0.26}\left(\Delta t_{\mathrm{TOR}, W 1}\right)^{2.27 \pm 0.09} .
$$

Given the mean value of $\Delta t_{\mathrm{BLR}} / \Delta t_{\mathrm{TOR}, 3 \mu \mathrm{m}} \sim 0.23$ for the PG quasars and $\Delta t_{\mathrm{TOR}, \mathrm{K}} / \Delta t_{\mathrm{TOR}, W 1} \sim 0.6$ from our fits of the Koshida et al. (2014) measurements (Section 4.2), we find $R_{\mathrm{BLR}} / R_{\mathrm{TOR}, K} \sim 0.4$. This result statistically confirms the values obtained in the three cases discussed above. It suggests that the BLR region is only slightly smaller than the size of the $K$-band dust emission, providing strong support for the picture proposed by Baskin \& Laor (2018) that large graphite dust grains might survive down to $R_{\mathrm{BLR}}$ and that the very hot dust emission comes from regions very close to the BLR.

\section{Summary}

We present the first statistical mid-IR dust reverberation mapping study of the quasar torus with an innovative usage of the long-term time-series data from the mid-IR WISE/NEOWISE 
mission and several optical transient surveys (CRTS, ASAS-SN, and PTF) over a timescale of about 8 yr. Compared with previous dedicated targeted observations, the data in these public archives have various complications and uncertainties. We have developed procedures to maximize the usefulness of these data and characterize the dust reverberation signals by comparing the mid-IR and optical light curves with a simple linear model. The success of this approach has been demonstrated by our detections of mid-IR dust lags to the optical variation signals in 67 out of $87 z<0.5$ PG quasars. Most of the remaining quasars have data quality issues or featureless mid-IR light curves, making analysis ambiguous. Our key results for AGN variability at $1-3 \mu \mathrm{m}$ are as follows.

1. The majority of PG quasars $(\sim 77 \%)$ have convincing dust reverberation signals with time-lags that follow the expected $\Delta t \propto L_{\mathrm{AGN}}^{0.5}$ relation. For the WISE W1 and $W 2$ filter bandpasses, we find

$$
\Delta t_{\text {torus }, W 1} / \text { day }=10^{2.10 \pm 0.06}\left(L_{\mathrm{AGN}, \mathrm{SED}} / 10^{11} L_{\odot}\right)^{0.47 \pm 0.06}
$$

and

$$
\Delta t_{\text {torus }, W 2} / \text { day }=10^{2.20 \pm 0.06}\left(L_{\mathrm{AGN}, \mathrm{SED}} / 10^{11} L_{\odot}\right)^{0.45 \pm 0.05} \text {. }
$$

Combined with previous studies in the near-IR, the AGN IR time-lags share the same scaling relation with AGN luminosity over 4 orders of magnitude, indicating that similar circumnuclear dust structures are common to AGNs.

2. By combining our mid-IR results of PG quasars and previous $K$-band analysis of Seyfert 1 nuclei by Koshida et al. (2014), we provide the first multiwavelength torus size constraints. Assuming the same $\Delta t \propto L_{\mathrm{AGN}}^{0.5}$ relation for different bands, we find average time-lag ratios of $\Delta t_{\mathrm{K}}: \Delta t_{\mathrm{W} 1}: \Delta t_{\mathrm{W} 2} \sim$ $0.6: 1.0: 1.2\left(\lambda_{\text {rest }} \sim 2.1,2.9,4.0 \mu \mathrm{m}\right)$.

3 . With the variability amplitudes derived from reverberation model fitting, it is possible to put some crude constraints on surface density profiles of AGN-heated hot dust grains. We find $\Sigma(r) \propto r^{0.3}$ at the $K$ band, $\Sigma(r) \propto r^{-0.7}$ at the $W 1$ band, and $\Sigma(r) \propto r^{-1.0}$ at the W2 band, indicating the concentration of the hottest dust grains in the innermost regions of the torus.

4. For the same AGN luminosity, the mid-IR emission region sizes of dust-deficient quasars are only $60 \%-70 \%$ of those found for normal quasars. In addition, their relative size differences in the WISE W1 and W2 bands are smaller, possibly indicating compact dust structures. Meanwhile, the differences between the optical and midIR variability amplitudes are smaller for HDD quasars than for normal quasars, indicating a smaller amount of dust reprocessing the accretion disk emission. On the other hand, there is no difference in the ratio of mid-IR to optical variability amplitude between WDD and normal quasars. These results are roughly consistent with their SED features and support their different torus structures, as argued by Lyu et al. (2017).

5. Using previous measurements of BLR size in the literature, the mean value of $R_{\mathrm{BLR}} / R_{\text {torus, } W 1} \sim 0.23 \pm 0.10$. Given that the relative IR time-lags between the $W I$ and $K$ bands, $R_{\mathrm{BLR}} / R_{\text {torus }, K} \sim 0.4$, indicating that the dust torus is located just outside of the AGN BLRs.

With the multi-epoch IR data from Spitzer and WISE, we also studied the AGN variability behavior at $10-24 \mu \mathrm{m}$ and explored its relationship to that at 3-5 $\mu \mathrm{m}$. The most important results are as follows.

1. With very few exceptions, significant AGN IR variability at $\lambda \gtrsim 10 \mu \mathrm{m}$ is only found among blazars and/or FSRQs. Considering the limited number of $24 \mu \mathrm{m}$ observations, it is likely that all of these sources vary in the mid-IR. In contrast with blazars and FSRQs, we have found only one SSRQ that is probably variable at $24 \mu \mathrm{m}$. Such a difference can be explained under the radio-loud AGN unification scheme. Since SSRQs have larger inclination angles to our line of sight compared with FSRQs, realistic beaming effects greatly enhance the variability of the latter population.

2. Besides blazars and FSRQs, the vast majority of AGNs do not show variability at $24 \mu \mathrm{m}$. That is, the IR variability amplitude decreases quickly as a function of wavelength. Compared with the $W 1$ band, the $W 2$ band variability is reduced to $90 \%$, and the $24 \mu \mathrm{m}$ is less than $10 \%$. Given the fact that the typical Wl-band variation rms is $\lesssim 0.1$ mag for most quasars, the corresponding flux change at $\sim 24 \mu \mathrm{m}$ is no more than $0.01 \mathrm{mag}$. Only in very rare cases is a possible dust reverberation signal detected (e.g., PG 1535+547).

We thank the referee, Makoto Kishimoto, for his constructive report.

This work was supported by NASA grants NNX13AD82G and 1255094 .

This publication has made use of data products from the Widefield Infrared Survey Explorer, which is a joint project of the University of California, Los Angeles, and the Jet Propulsion Laboratory/California Institute of Technology, funded by the National Aeronautics and Space Administration. This publication also makes use of data products from NEOWISE, which is a project of the Jet Propulsion Laboratory/California Institute of Technology, funded by the Planetary Science Division of the National Aeronautics and Space Administration. This work is based in part on archival data obtained with the Spitzer Space Telescope, which is operated by the Jet Propulsion Laboratory, California Institute of Technology, under a contract with NASA. Support for this work was provided by an award issued by JPL/Caltech.

The CSS survey is funded by the National Aeronautics and Space Administration under grant No. NNG05GF22G issued through the Science Mission Directorate Near-Earth Objects Observations Program. The CRTS survey is supported by the U.S. National Science Foundation under grants AST-0909182 and AST-1313422. The ASAS-SN is supported by the Gordon and Betty Moore Foundation through grant GBMF5490 to the Ohio State University and NSF grant AST-1515927. Development of ASAS-SN has been supported by NSF grant AST0908816, the Mt. Cuba Astronomical Foundation, the Center for Cosmology and Astro Particle Physics at the Ohio State University, the Chinese Academy of Sciences South America Center for Astronomy (CASSACA), the Villum Foundation, and George Skestos. Data from the Steward Observatory spectropolarimetric monitoring project were used. This program is supported by Fermi Guest Investigator grants NNX08AW56G, NNX09AU10G, NNX12AO93G, and NNX15AU81G. We also acknowledge with thanks the 3C 273 observations from the AAVSO International Database contributed by observers worldwide and used in this research. 
This work is based in part on observations made with the Spitzer Space Telescope, which is operated by the Jet Propulsion Laboratory, California Institute of Technology, under a contract with NASA. The Combined Atlas of Sources with Spitzer IRS Spectra (CASSIS) is a product of the IRS instrument team, supported by NASA and JPL.

Software: SExtractor (Bertin \& Arnouts 1996), DAOPHOT package (Stetson 1987), IRAF (Tody 1986, 1993), MPFIT (Markwardt 2009), JAVELIN (Zu et al. 2013), Matplotlib (Hunter 2007).

\section{Appendix A Photometric Stability}

None of the CRTS, ASAS-SN, and WISE/NEOWISE missions have put AGN variability study as their main science objective; thus, we need to assess the noise characteristics of their photometric measurements. For our time-series analysis, a clear idea of the instrument photometric stability is of paramount importance. With careful selections of photometric standard stars in the optical and mid-IR bands, we construct the light curves of nonvariable sources and explore the systematics and noise characteristics in this appendix.

\section{A.1. Optical Data from CRTS and ASAS-SN}

From the Optical and UV Spectrophotometric Standard Stars website at ESO, ${ }^{12}$ we have selected 15 stars to study the optical photometric stability of the CRTS and ASAS-SN data. Besides GD 108, a subdwarf, all of these stars are white dwarfs. We have compiled their optical measurements from the CRTS and ASAS-SN archives and constructed the corresponding optical light curves. Since the CRTS data were taken without a photometric filter, we matched the CRTS light curve to those derived from ASAS-SN by introducing a constant offset. We also rejected photometry outliers that showed a discrepancy of $>0.5 \mathrm{mag}$ from the average values. The final light curves are plotted in Figure 16.

We calculate the mean magnitudes and rms offsets of the CRTS and ASAS-SN light curves separately and summarize the results in Table 10. Besides the uncertainties caused by standard photometry measurements, any issues with system stability can further increase the observed magnitude rms offset, and these values could be related as

$$
\mathrm{rms}_{M-\bar{M}}^{2}=\mathrm{rms}_{\Delta M}^{2}+\sigma_{\text {s.s. }}^{2}
$$

On average, we get $\sigma_{\text {s.s. }} \sim 0.018 \mathrm{mag}$ for ASAS-SN data and $\sigma_{\text {s.s. }} \sim 0.023$ mag for CRTS data.

\section{A.2. Mid-IR from WISE/NEOWISE}

With the WISE/NEOWISE catalogs, we examined the photometric stability of the $W 1$ and $W 2$ bands as a function of time. Fourteen mid-IR standard stars from the Spitzer/IRAC primary calibrators $^{13}$ and WISE (WI and W2) calibration stars ${ }^{14}$ were chosen for this purpose. The constructed mid-IR light curves can be seen in Figure 17. We summarize the rms magnitude offsets and errors of the $W 1$ and $W 2$ bands during the WISE and NEOWISE missions in Table 11.

With Equation (34), we get $\sigma_{\text {s.s. }} \sim 0.029 \mathrm{mag}$ for WISE and $0.016 \mathrm{mag}$ for NEOWISE. Since we have averaged the 10-20 observations for the mid-IR light-curve construction (Section 2.2.2), the contribution to the measurement uncertainties from the system instability is expected to be $<0.009 \mathrm{mag}$ for WISE and $<0.005 \mathrm{mag}$ for NEOWISE.

\footnotetext{
12 http://www.eso.org/sci/observing/tools/standards/spectra/stanlis.html
}

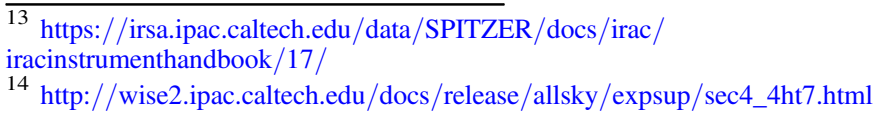



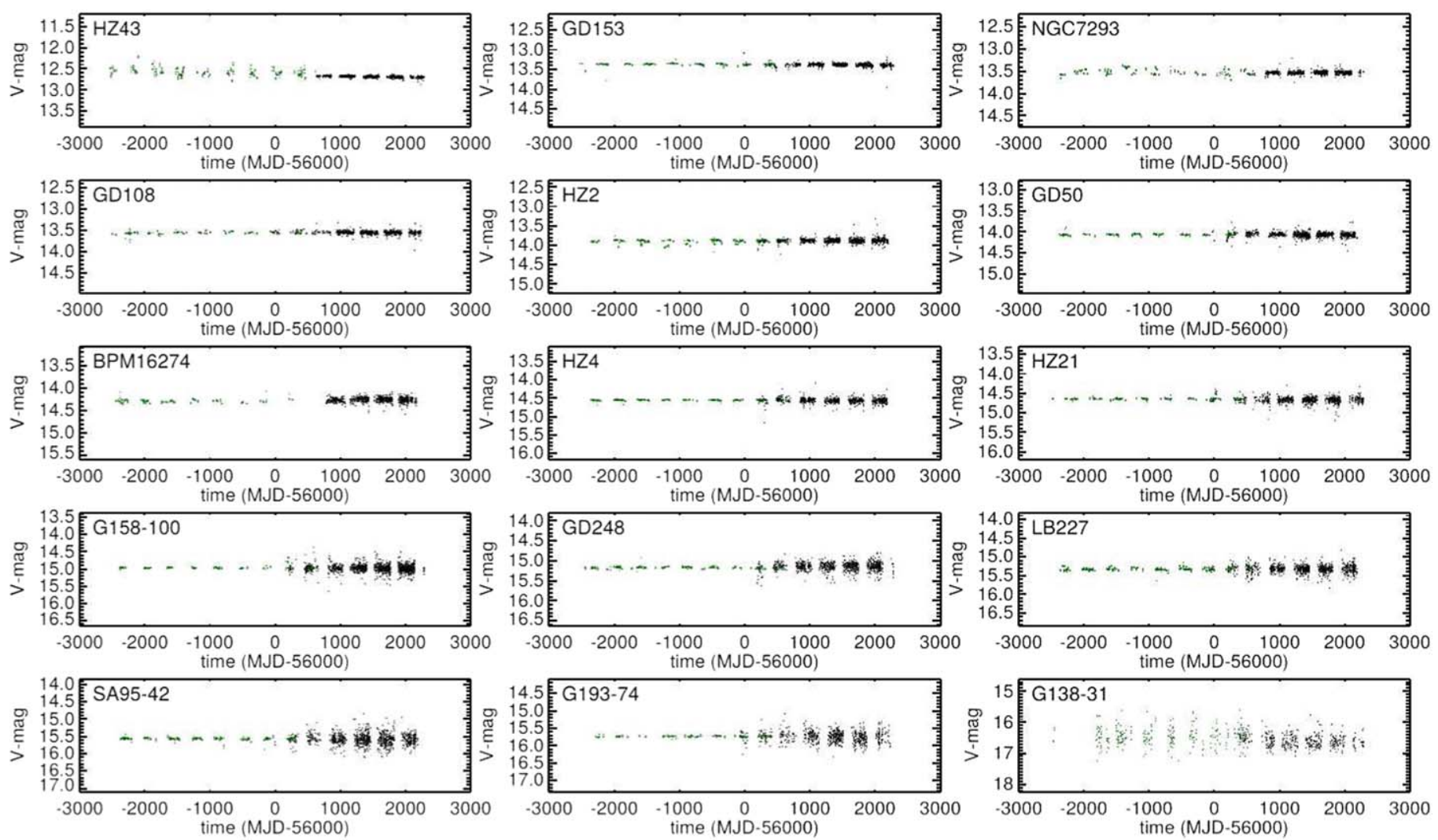

Figure 16. Optical light curves of 15 optical spectrophotometric standard stars. The CRTS data are in green, and the ASAS-SN data are in black.

Table 10

Measurements of Optical Standard Stars

\begin{tabular}{|c|c|c|c|c|c|c|c|}
\hline \multirow{2}{*}{$\begin{array}{l}\text { Name } \\
\text { (1) }\end{array}$} & \multirow{2}{*}{$\begin{array}{l}M_{V} \\
\\
(2)\end{array}$} & \multicolumn{3}{|c|}{ CRTS } & \multicolumn{3}{|c|}{ ASAS-SN } \\
\hline & & $\begin{array}{l}\bar{M} \\
(3)\end{array}$ & $\begin{array}{l}\mathrm{rms}_{M-\bar{M}} \\
\quad(4)\end{array}$ & $\begin{array}{l}\operatorname{rms}_{\Delta M} \\
\quad(5)\end{array}$ & $\begin{array}{l}\bar{M} \\
(6)\end{array}$ & $\begin{array}{r}\mathrm{rms}_{M-\bar{M}} \\
\quad(7)\end{array}$ & $\begin{array}{c}\operatorname{rms}_{\Delta M} \\
\quad(8)\end{array}$ \\
\hline GD 153 & 13.35 & 13.371 & 0.036 & 0.011 & 13.384 & 0.041 & 0.022 \\
\hline NGC 7293 & 13.51 & 13.527 & 0.065 & 0.013 & 13.534 & 0.035 & 0.030 \\
\hline GD 108 & 13.56 & 13.551 & 0.034 & 0.012 & 13.548 & 0.039 & 0.027 \\
\hline HZ 2 & 13.86 & 13.902 & 0.051 & 0.012 & 13.882 & 0.053 & 0.034 \\
\hline $\mathrm{HZ} 4$ & 14.52 & 14.555 & 0.016 & 0.017 & 14.568 & 0.066 & 0.048 \\
\hline HZ 21 & 14.68 & 14.646 & 0.022 & 0.019 & 14.670 & 0.077 & 0.054 \\
\hline G158-100 & 14.89 & 14.968 & 0.022 & 0.018 & 14.980 & 0.109 & 0.076 \\
\hline GD 248 & 15.09 & 15.170 & 0.025 & 0.020 & 15.146 & 0.102 & 0.083 \\
\hline LB 227 & 15.34 & 15.326 & 0.038 & 0.019 & 15.324 & 0.101 & 0.083 \\
\hline SA $95-42$ & 15.61 & 15.567 & 0.040 & 0.023 & 15.581 & 0.156 & 0.123 \\
\hline
\end{tabular}

Note. Col. (1): star name. Col. (2): $V$-band magnitude given in the ESO standard star list. Col. (3): mean magnitude of all CRTS measurements; an offset is included to match the CRTS light curve. Col. (4): rms value for all of the magnitude deviations relative to the mean magnitude for CRTS data. Col. (5): rms value for all magnitude errors reported in the CRTS catalog. Cols. (6)-(8): similar to cols. (3)-(5) but for ASAS-SN data. 

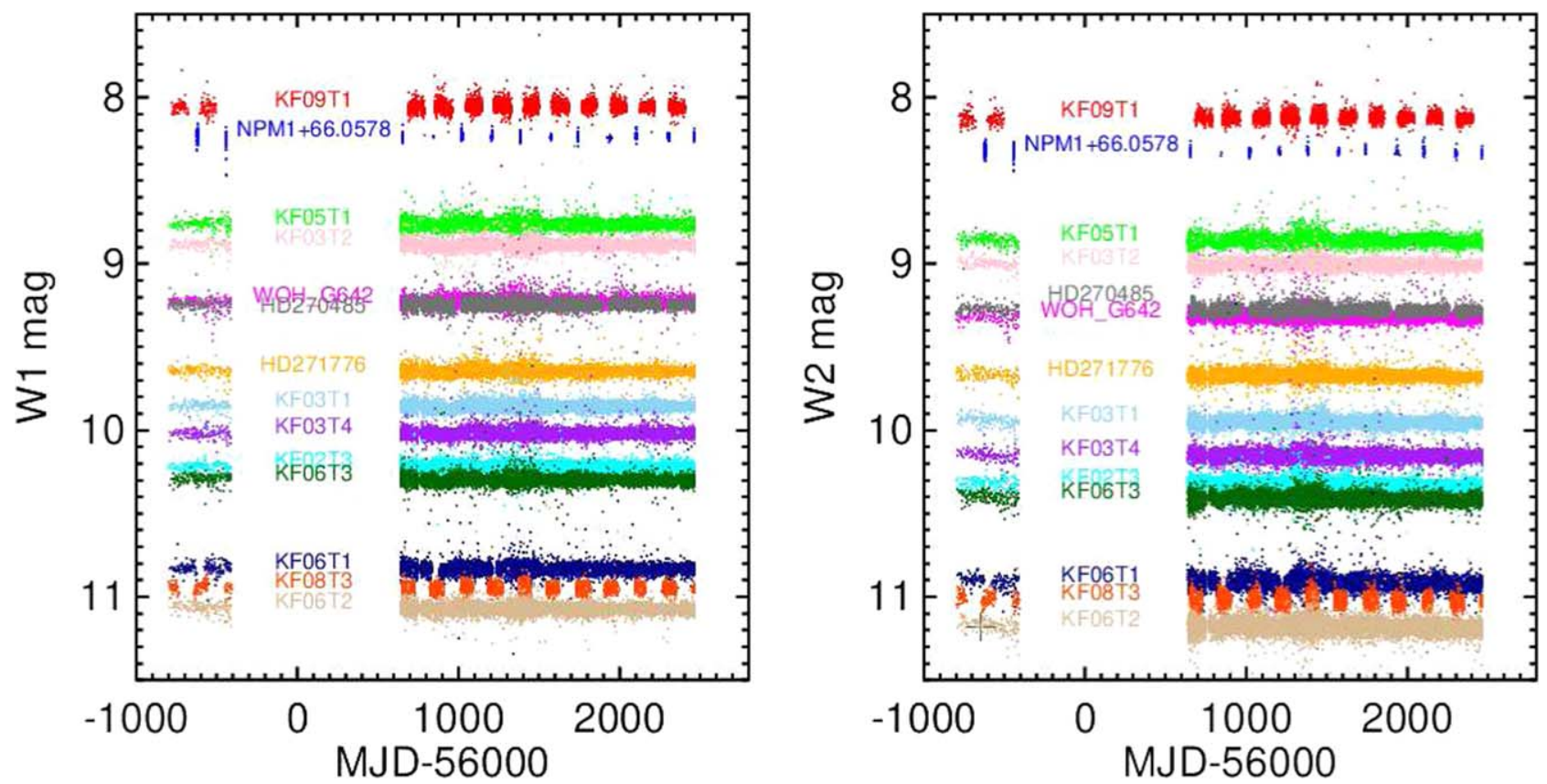

Figure 17. WISE W1 (left) and W2 (right) light curves of 14 IR calibration standard stars.

Table 11

Measurements of Mid-IR Standard Stars

\begin{tabular}{|c|c|c|c|c|c|c|c|c|c|c|}
\hline \multirow{3}{*}{$\begin{array}{l}\text { Name } \\
\text { (1) }\end{array}$} & \multicolumn{5}{|c|}{ W1 Band } & \multicolumn{5}{|c|}{ W2 Band } \\
\hline & \multirow[b]{2}{*}{$\begin{array}{l}\bar{M} \\
(2)\end{array}$} & \multicolumn{2}{|c|}{ ALLWISE } & \multicolumn{2}{|c|}{ NEOWISE } & \multirow[b]{2}{*}{$\begin{array}{l}\bar{M} \\
(7)\end{array}$} & \multicolumn{2}{|c|}{ ALLWISE } & \multicolumn{2}{|c|}{ NEOWISE } \\
\hline & & $\begin{array}{r}\mathrm{rms}_{M-\bar{M}} \\
\text { (3) }\end{array}$ & $\begin{array}{l}\mathrm{rms}_{\Delta M} \\
\quad(4)\end{array}$ & $\underset{(5)}{\operatorname{rms}_{M-\bar{M}}}$ & $\begin{array}{l}\mathrm{rms}_{\Delta M} \\
\quad(6)\end{array}$ & & $\begin{array}{l}\mathrm{rms}_{M-\bar{M}} \\
\quad(8)\end{array}$ & $\begin{array}{c}\operatorname{rms}_{\Delta M} \\
(9)\end{array}$ & $\begin{array}{l}\mathrm{rms}_{M-\bar{M}} \\
\quad(10)\end{array}$ & $\begin{array}{c}\mathrm{rms}_{\Delta M} \\
\quad(11)\end{array}$ \\
\hline KF 09T1 & 8.055 & 0.038 & 0.024 & 0.022 & 0.016 & 8.123 & 0.037 & 0.021 & 0.022 & 0.016 \\
\hline NPM $1+66.0578$ & 8.237 & 0.041 & 0.024 & 0.020 & 0.016 & 8.323 & 0.032 & 0.021 & 0.020 & 0.016 \\
\hline KF 05T1 & 8.767 & 0.035 & 0.024 & 0.019 & 0.016 & 8.863 & 0.027 & 0.022 & 0.019 & 0.016 \\
\hline KF $03 \mathrm{~T} 2$ & 8.891 & 0.029 & 0.024 & 0.020 & 0.016 & 9.007 & 0.033 & 0.022 & 0.020 & 0.016 \\
\hline HD 271776 & 9.647 & 0.032 & 0.024 & 0.023 & 0.016 & 9.671 & 0.034 & 0.022 & 0.023 & 0.016 \\
\hline WOH_G 642 & 9.229 & 0.042 & 0.024 & 0.021 & 0.016 & 9.319 & 0.036 & 0.022 & 0.021 & 0.016 \\
\hline HD 270485 & 9.248 & 0.030 & 0.025 & 0.031 & 0.016 & 9.277 & 0.052 & 0.022 & 0.031 & 0.016 \\
\hline KF 03T1 & 9.858 & 0.033 & 0.025 & 0.022 & 0.016 & 9.952 & 0.049 & 0.023 & 0.022 & 0.016 \\
\hline KF 03T4 & 10.019 & 0.034 & 0.025 & 0.021 & 0.016 & 10.155 & 0.035 & 0.023 & 0.021 & 0.016 \\
\hline KF $02 \mathrm{~T} 3$ & 10.223 & 0.037 & 0.025 & 0.028 & 0.017 & 10.332 & 0.035 & 0.025 & 0.028 & 0.017 \\
\hline KF 06T3 & 10.298 & 0.030 & 0.025 & 0.022 & 0.017 & 10.410 & 0.032 & 0.025 & 0.022 & 0.017 \\
\hline KF 06T1 & 10.832 & 0.036 & 0.026 & 0.023 & 0.018 & 10.912 & 0.039 & 0.027 & 0.023 & 0.018 \\
\hline KF $08 \mathrm{~T} 3$ & 10.946 & 0.033 & 0.027 & 0.021 & 0.018 & 11.020 & 0.039 & 0.028 & 0.021 & 0.018 \\
\hline KF 06T2 & 11.072 & 0.031 & 0.026 & 0.031 & 0.018 & 11.179 & 0.042 & 0.029 & 0.031 & 0.018 \\
\hline
\end{tabular}

Note. Col. (1): star name. Col. (2): average W1 magnitude of all WISE/NEOWISE measurements. Col. (3): rms magnitude offset relative to the mean W1 magnitude for measurements taken during the ALLWISE mission. Col. (4): rms value of all Wl magnitude errors reported in the ALLWISE Multiepoch Photometry Table. Cols. (5)-(6): similar to cols. (3)-(4) but for W1 measurements taken during the NEOWISE mission. Cols. (7)-(11): similar to cols. (2)-(6) but for W2-band measurements. 


\section{Appendix B \\ A Simple Model for Relating Time-Lags to the Torus Structures}

We As shown in Figure 18, we consider a thin dust shell with a half opening angle $\Omega_{\mathrm{TOR}}$ that surrounds the accretion disk. We also assume that a single WISE band traces the IR emission from dust at some similar distance, $R$, to the accretion disk. Placing the axis of symmetry along the $z$-axis, we have a dusty layer with $r=R, \theta=\left[\Omega_{\mathrm{TOR}}, \pi-\Omega_{\mathrm{TOR}}\right]$, and $\phi=[0$, $2 \pi$ ). The observer is located at an observing angle of $\Omega_{\mathrm{LOS}}$ to the $z$-axis and parallel to the $z-y$ plane.

Now imagine a plane that is perpendicular to the observing angle and also tangent to the dusty sphere, which can be described by the function

$$
\left[\sin \Omega_{\mathrm{LOS}}\right] y+\left[\cos \Omega_{\mathrm{LOS}}\right] z-R=0 .
$$

It takes time, $R / c$, for emission from the accretion disk to reach this plane.

For a point on the dusty sphere with coordinates $(x, y, z)=R(\sin \theta \cos \phi, \sin \theta \sin \phi, \cos \theta)$, the light travel distance would be

$$
\begin{aligned}
l= & R+\left|R\left[\sin \Omega_{\mathrm{LOS}} \sin \theta \sin \phi+\cos \Omega_{\mathrm{LOS}} \cos \theta-1\right]\right| \\
& =R\left[2-\sin \Omega_{\mathrm{LOS}} \sin \theta \sin \phi-\cos \Omega_{\mathrm{LOS}} \cos \theta\right] .
\end{aligned}
$$

An average time-lag of all elements on that sphere is given by

$$
\begin{gathered}
\langle t\rangle=\frac{\int w l d s}{c \int d s} \\
=\frac{\int_{0}^{2 \pi} \int_{\Omega_{\mathrm{TOR}}}^{\pi-\Omega_{\mathrm{TOR}}} w l R \sin \theta d \theta d \phi}{c \int_{0}^{2 \pi} \int_{\Omega_{\mathrm{TOR}}}^{\pi-\Omega_{\mathrm{TOR}}} R \sin \theta d \theta d \phi},
\end{gathered}
$$

where $w$ is the weight of each element, which is dependent on the dust optical depth in the IR band along the line of sight and the local strength of dust emission. If the emission is optically thin and homogeneously distributed, we have $w=1$, so

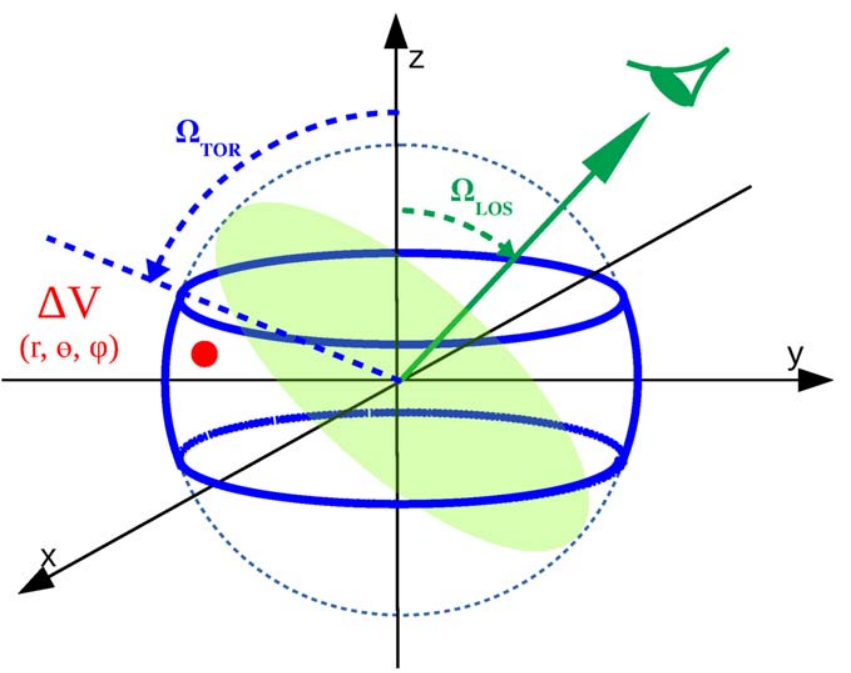

Figure 18. Geometry of a dusty shell with a radius $r$ from the origin (accretion disk) and a half opening angle $\Omega_{\mathrm{TOR}}$. The observer has a viewing angle $\Omega_{\mathrm{LOS}}$.

If the dusty torus is extremely optically thick, we can only see flux from the edge of the structure that is exposed to the observer. When the observer is looking through the torus opening angle (type $1 ; \Omega_{\mathrm{LOS}}<\Omega_{\mathrm{TOR}}$ ), the time-lag can be easily calculated to be

$$
\Delta t=\frac{R}{c}\left(1-\cos \Omega_{\mathrm{LOS}} \cos \Omega_{\mathrm{TOR}}\right)
$$

It is straightforward to prove that the time-lag in this situation will always be shorter than the optically thin case for the same $R$.

In true physical situations where the torus dust is not perfectly optically thin or thick in the mid-IR, we should have

$$
\frac{R}{c}>\Delta t>\frac{R}{c}\left(1-\cos \Omega_{\mathrm{LOS}} \cos \Omega_{\mathrm{TOR}}\right) .
$$

Knowing the fraction of type- 2 objects in the quasar population $\left(\sim 0.5-0.6\right.$; Reyes et al. 2008), we have roughly $\Omega_{\mathrm{TOR}}=\pi / 3$

$$
\langle t\rangle=\frac{\int_{0}^{2 \pi} \int_{\Omega_{\mathrm{TOR}}}^{\pi-\Omega_{\mathrm{TOR}}} R\left[2-\sin \Omega_{\mathrm{LOS}} \sin \theta \sin \phi-\cos \Omega_{\mathrm{LOS}} \cos \theta\right] R \sin \theta d \theta d \phi}{c \int_{0}^{2 \pi} \int_{\Omega_{\mathrm{TOR}}}^{\pi-\Omega_{\mathrm{TOR}}} R \sin \theta d \theta d \phi}
$$

$$
=\frac{2 R}{c} \text {. }
$$

Consequently, the average time-lag between the surrounding dusty torus shell and the accretion disk is

$$
\Delta t=\frac{R}{c}
$$

This means that the time-lag is not dependent on the viewing angle in the optically thin case. and

$$
\frac{R}{c}>\Delta t \gtrsim 0.5 \frac{R}{c} .
$$

In other words, a naïve size estimation from $c \Delta t$ could slightly underestimate the true physical size up to a factor of two. For a single ring with $\theta=\theta_{0}$, the range of time-lags, $\Delta t$, is given by

$$
\max (\Delta t)-\min (\Delta t)=\frac{2 R}{c} \sin \Omega_{\mathrm{LOS}} \sin \theta_{0} .
$$



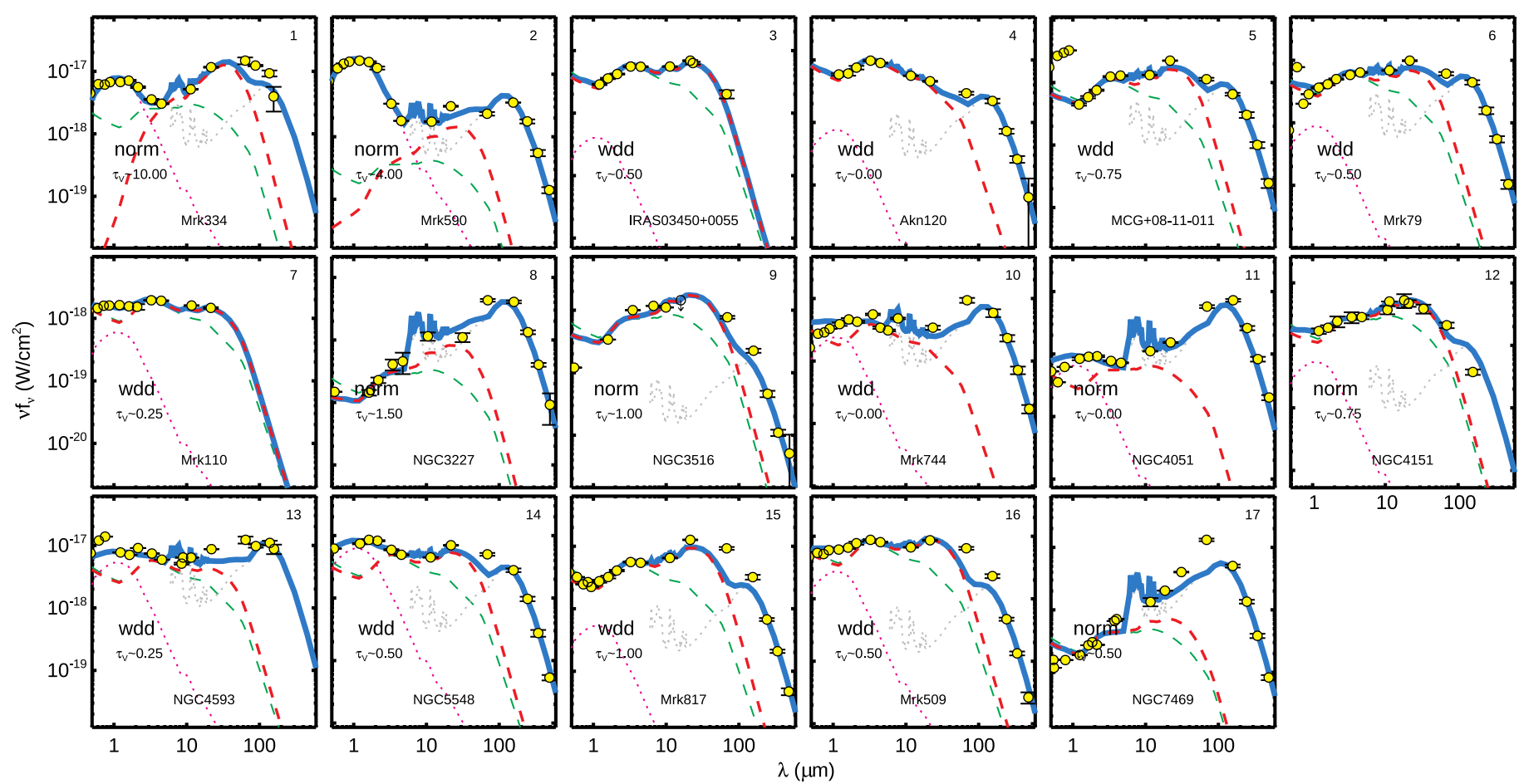

Figure 19. Best-fit results for the Seyfert 1 nuclei studied in Koshida et al. (2014). Photometric data points are shown as yellow dots. The SED model (blue solid lines) is composed of the AGN component (red dashed lines), stellar component (magenta dotted lines), and far-IR star formation component (gray dotted lines). We also plot the suggested intrinsic AGN template (green dashed lines) for each object from our SED fittings to compare with the observed SED.

For a complete shell,

$$
\max (\Delta t)-\min (\Delta t)=\frac{2 R}{c} \cos \left(\Omega_{\mathrm{TOR}}-\Omega_{\mathrm{LOS}}\right) .
$$

Finally, the standard deviation of the time-lag $\Delta t$ for a dusty shell is

$$
\sigma(\Delta t)=\frac{R}{c} \sqrt{\frac{1}{3} \cos ^{2} \Omega_{\mathrm{TOR}}+\frac{1}{2} \sin ^{2} \Omega_{\mathrm{LOS}} \sin ^{2} \Omega_{\mathrm{TOR}}} .
$$

This can introduce a smoothing effect on the integrated IR reverberation light curves, whose strength is proportional to the torus size (or time lag $\Delta t$ ).

\section{Appendix C \\ A Revisit of the Koshida et al. Sample}

\section{C.1. SED Analysis}

For consistency with the luminosity estimates for the PG sample, we examined the broadband IR SEDs of the Seyfert 1 sample presented by Koshida et al. (2014) with the decomposition model introduced in Lyu \& Rieke (2018). The total IR emission is assumed to be a linear combination of the contributions from three components: AGN-heated dust, near-IR starlight, and mid-to-farIR emission from $\mathrm{H}$ II regions within the host galaxy. To reduce the ambiguity of interpretation, we have used well-tested empirical templates to describe each component. For the AGN templates, we first used the three types of intrinsic AGN SEDs proposed in Lyu et al. (2017) to represent the intrinsic variations of the torus. We also allow obscuration by an IR optically thin extended dust distribution of large grains and the corresponding IR reprocessed emission. The latter component has been shown to be a valid explanation for the AGN polar dust emission seen by mid-IR interferometry analysis (see details in Lyu \& Rieke 2018). We used $\chi^{2}$ minimization to determine the final best-fit model. The

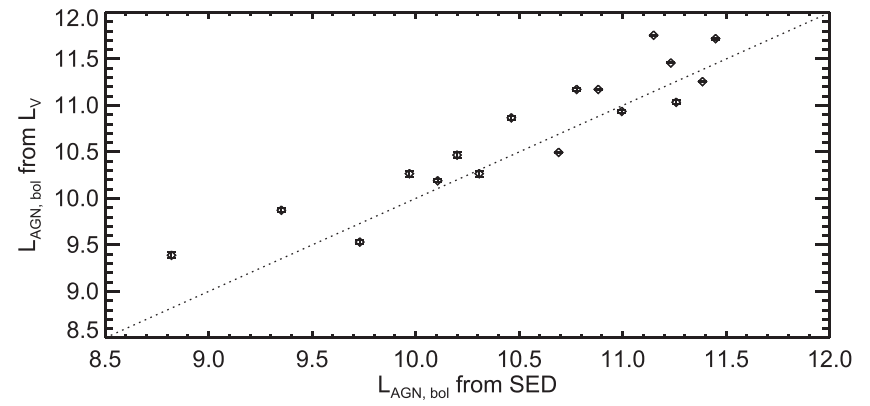

Figure 20. The AGN luminosity estimation comparison between SED fitting and $V$-band photometry from Koshida et al. (2014). The dotted line is the 1:1 relation.

dust-deficient AGN template was selected only when it improved the $\chi^{2}$ value by a factor greater than 2 compared with the normal AGN template.

The results can be seen in Figure 19. About half of the Koshida et al. (2014) sample has been identified as WDD AGNs, and the other half consists of normal AGNs. With the derived intrinsic AGN SEDs, we calculated the AGN bolometric luminosities following Section 4.2.1 and summarized the results in Table 12. As shown in Figure 20, the results are consistent with those converted from the observed $V$-band measurements provided in Koshida et al. (2014), yielding an average $L_{\mathrm{AGNbol}, \mathrm{SED}} / L_{\mathrm{AGN} \text { bol, } \mathrm{V}}=0.98 \pm 0.03$.

\section{C.2. Time-lag Measurements}

To determine if our time-lag measurements are significantly different from those determined by traditional CCF analysis, we apply the model introduced in Section 3.2 to fit the highcadence light curves of 17 Seyfert 1 nuclei in Koshida et al. (2014) and present our measurements of the $K$-band time lags 


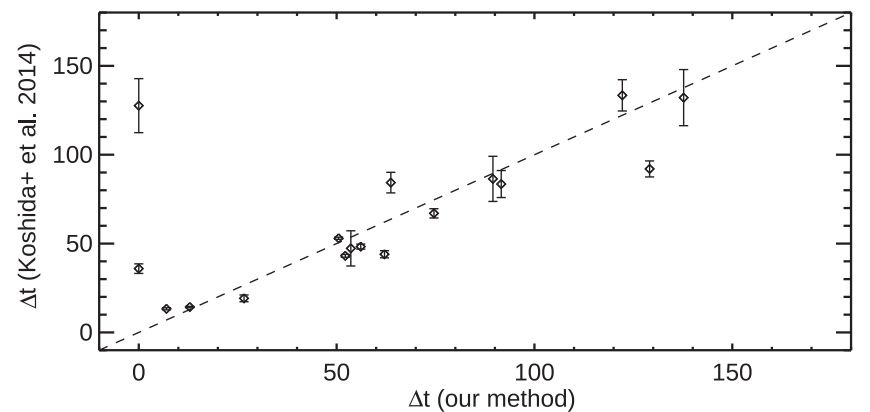

Figure 21. The $K$-band time-lag comparison between our model and the Koshida et al. (2014) method. The dotted line is the 1:1 relation.

Table 12

New Time-lag Measurements of the Koshida et al. (2014) Seyfert 1 Sample

\begin{tabular}{lccccr}
\hline \hline \multirow{2}{*}{ Name } & Type & \multicolumn{2}{c}{$\log \left(L_{\text {AGN,bol }} / L_{\odot}\right)$} & \multirow{2}{*}{$\Delta t$ day $^{-1}$} & \multirow{2}{*}{ AMP } \\
\cline { 3 - 4 } & & $(V$-band $)$ & $($ SED $)$ & & \\
\hline Mrk 334 & Norm & 11.17 & 10.88 & 137.7 & 5.1 \\
Mrk 590 & Norm & 10.46 & 10.20 & 0 & 6.2 \\
IRAS 03450+0055 & WDD & 11.46 & 11.23 & 129.1 & 6.2 \\
Akn 120 & WDD & 11.87 & 11.14 & 0 & 4.0 \\
MCG +08-11-011 & WDD & 11.76 & 11.25 & 89.5 & 9.5 \\
Mrk 79 & WDD & 10.93 & 10.99 & 74.6 & 2.1 \\
Mrk 110 & Norm & 11.17 & 10.77 & 63.7 & 2.8 \\
NGC 3227 & Norm & 9.85 & 9.35 & 7.0 & 5.3 \\
NGC 3516 & Norm & 10.26 & 10.30 & 53.6 & 6.8 \\
Mrk 744 & WDD & 9.48 & 9.73 & 26.6 & 19.9 \\
NGC 4051 & Norm & 9.30 & 8.82 & 12.9 & 6.4 \\
NGC 4151 & Norm & 10.26 & 9.97 & 52.2 & 4.2 \\
NGC 4593 & WDD & 10.17 & 10.10 & 62.1 & 6.9 \\
NGC 5548 & WDD & 10.49 & 10.69 & 50.5 & 4.9 \\
Mrk 817 & Norm & 11.26 & 11.38 & 91.6 & 6.7 \\
Mrk 590 & WDD & 11.71 & 11.44 & 122.2 & 2.4 \\
NGC 7469 & Norm & 10.87 & 10.46 & 56.1 & 5.1 \\
\hline
\end{tabular}

in Table 12. In Figure 21, we compare the results from these two approaches. Except for Mrk 590 and Akn 120, most measurements are consistent with each other, and the average difference is $<10 \%$. This suggests that $R_{K} / R_{W 1} \sim 0.6$ is not caused by the systematics from the different measurement approaches.

\section{ORCID iDs}

Jianwei Lyu (吕建伟) (10) https://orcid.org/0000-0002-6221-1829 George H. Rieke (iD https://orcid.org/0000-0003-2303-6519

\section{References}

Almeyda, T., Robinson, A., Richmond, M., Vazquez, B., \& Nikutta, R. 2017, ApJ, 843, 3

Antonucci, R. 1993, ARA\&A, 31, 473

Barvainis, R. 1987, ApJ, 320, 537

Barvainis, R. 1992, ApJ, 400, 502

Baskin, A., \& Laor, A. 2018, MNRAS, 474, 1970

Beichman, C. A., Neugebauer, G., Habing, H. J., Clegg, P. E., \& Chester, T. J. 1988, Infrared Astronomical Satellite (IRAS) Catalogs and Atlases Vol. 1 (Washington, DC: NASA)

Berriman, G., Schmidt, G. D., West, S. C., \& Stockman, H. S. 1990, ApJS, 74,869

Bertin, E., \& Arnouts, S. 1996, A\&AS, 117, 393
Blandford, R. D., \& McKee, C. F. 1982, ApJ, 255, 419

Boroson, T. A., \& Green, R. F. 1992, ApJS, 80, 109

Carey, S. J. 2010, in Proc. 2010 STScI Calibration Workshop, ed. S. Deustua \& C. Oliveira (Baltimore, MD: STScI), 37

Clavel, J., Wamsteker, W., \& Glass, I. S. 1989, ApJ, 337, 236

Cohen, M. H., Lister, M. L., Homan, D. C., et al. 2007, ApJ, 658, 232

Cutri, R. M., Nelson, B. O., Francis, P. J., et al. 2002, in ASP Conf. Ser. 284, AGN Surveys, ed. R. F. Green, E. Ye. Khachikian, \& D. B. Sanders (San Francisco, CA: ASP), 127

Czerny, B., \& Hryniewicz, K. 2011, A\&A, 525, L8

Diamond-Stanic, A. M., Rieke, G. H., \& Rigby, J. R. 2009, ApJ, 698, 623

Draine, B. T., \& Lee, H. M. 1984, ApJ, 285, 89

Drake, A. J., Djorgovski, S. G., Mahabal, A., et al. 2009, ApJ, 696, 870

Edelson, R. A., \& Malkan, M. A. 1987, ApJ, 323, 516

Elvis, M., Wilkes, B. J., McDowell, J. C., et al. 1994, ApJS, 95, 1

Engelbracht, C. W., Blaylock, M., Su, K. Y. L., et al. 2007, PASP, 119, 994

Gallagher, S. C., Schmidt, G. D., Smith, P. S., et al. 2005, ApJ, 633, 71

Glass, I. S. 2004, MNRAS, 350, 1049

Glikman, E., Helfand, D. J., \& White, R. L. 2006, ApJ, 640, 579

Gordon, K. D., Rieke, G. H., Engelbracht, C. W., et al. 2005, PASP, 117, 503

Graham, M. J., Djorgovski, S. G., Drake, A. J., et al. 2014, MNRAS, 439, 703

Graham, M. J., Djorgovski, S. G., Drake, A. J., et al. 2017, MNRAS, 470, 4112

Hernán-Caballero, A., Hatziminaoglou, E., Alonso-Herrero, A., et al. 2016, MNRAS, 463, 2064

Hodge, J. A., Becker, R. H., White, R. L., et al. 2011, AJ, 142, 3

Homan, D. C. 2012, Int. J. Modern Phys. Conf. Ser., 8, 163

Hönig, S. F., Kishimoto, M., Gandhi, P., et al. 2010, A\&A, 515, A23

Houck, J. R., Roellig, T. L., van Cleve, J., et al. 2004, ApJS, 154, 18

Hunter, J. D. 2007, CSE, 9, 90

Jiang, Y.-F., Green, P. J., Greene, J. E., et al. 2017, ApJ, 836, 186

Kasliwal, V. P., Vogeley, M. S., \& Richards, G. T. 2017, MNRAS, 470, 3027

Kaspi, S., Smith, P. S., Netzer, H., et al. 2000, ApJ, 533, 631

Kawaguchi, T., \& Mori, M. 2011, ApJ, 737, 105

Kellermann, K. I., Condon, J. J., Kimball, A. E., et al. 2016, ApJ, 831, 168

Kellermann, K. I., \& Pauliny-Toth, I. I. K. 1981, ARA\&A, 19, 373

Kellermann, K. I., Sramek, R., Schmidt, M., et al. 1989, AJ, 98, 1195

Kelly, B. C., Bechtold, J., \& Siemiginowska, A. 2009, ApJ, 698, 895

Kelly, B. C., Becker, A. C., Sobolewska, M., et al. 2014, ApJ, 788, 33

Kim, D., Im, M., Kim, J. H., et al. 2015, ApJS, 216, 17

Kishimoto, M., Antonucci, R., Blaes, O., et al. 2008, Natur, 454, 492

Kishimoto, M., Hönig, S. F., Antonucci, R., et al. 2011, A\&A, 536, A78

Kishimoto, M., Hönig, S. F., Antonucci, R., et al. 2013, ApJL, 775, L36

Kishimoto, M., Hönig, S. F., Beckert, T., \& Weigelt, G. 2007, A\&A, 476, 713

Kishimoto, M., Hönig, S. F., Tristram, K. R. W., \& Weigelt, G. 2009, A\&A, 493, L57

Kochanek, C. S., Shappee, B. J., Stanek, K. Z., et al. 2017, PASP, 129, 104502

Koshida, S., Minezaki, T., Yoshii, Y., et al. 2014, ApJ, 788, 159

Kozłowski, S., Kochanek, C. S., Udalski, A., et al. 2010, ApJ, 708, 927

Laor, A., Baldi, R. D., \& Behar, E. 2019, MNRAS, 482, 5513

Laor, A., \& Draine, B. T. 1993, ApJ, 402, 441

Law, N. M., Kulkarni, S. R., Dekany, R. G., et al. 2009, PASP, 121, 1395

Lebouteiller, V., Barry, D. J., Spoon, H. W. W., et al. 2011, ApJS, 196, 8

Leipski, C., Meisenheimer, K., Walter, F., et al. 2013, ApJ, 772, 103

Lira, P., Arévalo, P., Uttley, P., McHardy, I., \& Breedt, E. 2011, MNRAS, 415, 1290

Lyu, J., \& Rieke, G. H. 2017, ApJ, 841, 76

Lyu, J., \& Rieke, G. H. 2018, ApJ, 866, 92

Lyu, J., Rieke, G. H., \& Shi, Y. 2017, ApJ, 835, 257

MacLeod, C. L., Ivezić, Ž, Kochanek, C. S., et al. 2010, ApJ, 721, 1014

Mainzer, A., Bauer, J., Cutri, R. M., et al. 2014, ApJ, 792, 30

Mandal, A. K., Rakshit, S., Kurian, K. S., et al. 2018, MNRAS, 475, 5330

Mao, P., Urry, C. M., Massaro, F., et al. 2016, ApJS, 224, 26

Markwardt, C. B. 2009, in ASP Conf. Ser. 411, Astronomical Data Analysis Software and Systems XVIII, ed. D. Durand, D. A. Bohlender, \& P. Dowler (San Francisco, CA: ASP), 251

Meléndez, M., Kraemer, S. B., Armentrout, B. K., et al. 2008, ApJ, 682, 94

Mor, R., Netzer, H., \& Elitzur, M. 2009, ApJ, 705, 298

Murphy, T., Mauch, T., Green, A., et al. 2007, MNRAS, 382, 382

Mushotzky, R. F., Edelson, R., Baumgartner, W., et al. 2011, ApJL, 743, L12

Nenkova, M., Sirocky, M. M., Ivezić, Ž, \& Elitzur, M. 2008, ApJ, 685, 147

Netzer, H. 2015, ARA\&A, 53, 365

Netzer, H., Shemmer, O., Maiolino, R., et al. 2004, ApJ, 614, 558

Neugebauer, G., \& Matthews, K. 1999, AJ, 118, 35

Oknyanskij, V. L., \& Horne, K. 2001, in ASP Conf. Ser. 224, Probing the Physics of Active Galactic Nuclei, ed. B. M. Peterson, R. W. Pogge, \& R. S. Polidan (San Francisco, CA: ASP), 149 
Peterson, B. M., Ferrarese, L., Gilbert, K. M., et al. 2004, ApJ, 613, 682

Pozo Nuñez, F., Haas, M., Chini, R., et al. 2014, A\&A, 561, L8

Pozo Nuñez, F., Ramolla, M., Westhues, C., et al. 2015, A\&A, 576, A73

Ramolla, M., Haas, M., Westhues, C., et al. 2018, A\&A, 620, A137

Reyes, R., Zakamska, N. L., Strauss, M. A., et al. 2008, AJ, 136, 2373

Rieke, G. H. 1972, ApJL, 176, L61

Rieke, G. H., \& Kinman, T. D. 1974, ApJL, 192, L115

Rigby, J. R., Diamond-Stanic, A. M., \& Aniano, G. 2009, ApJ, 700, 1878

Risaliti, G., \& Elvis, M. 2004, ASSL, 308, 187

Runnoe, J. C., Brotherton, M. S., \& Shang, Z. 2012, MNRAS, 426, 2677

Schartmann, M., Wada, K., Prieto, M. A., Burkert, A., \& Tristram, K. R. W. 2014, MNRAS, 445, 3878

Schmidt, G. D., Stockman, H. S., \& Smith, P. S. 1992, ApJL, 398, L57

Schmidt, M., \& Green, R. F. 1983, ApJ, 269, 352

Shappee, B. J., Prieto, J. L., Grupe, D., et al. 2014, ApJ, 788, 48

Shi, Y., Rieke, G. H., Hines, D. C., Gordon, K. D., \& Egami, E. 2007, ApJ, 655,781

Shi, Y., Rieke, G. H., Ogle, P. M., Su, K. Y. L., \& Balog, Z. 2014, ApJS, 214,23
Sloan, G. C., Herter, T. L., Charmandaris, V., et al. 2015, AJ, 149, 11 Smith, P. S. 2016, Galax, 4, 27

Smith, P. S., Schmidt, G. D., Allen, R. G., et al. 1997, ApJ, 488, 202

Soldi, S., Türler, M., Paltani, S., et al. 2008, A\&A, 486, 411

Stalevski, M., Fritz, J., Baes, M., Nakos, T., \& Popović, L. Č. 2012, MNRAS, 420, 2756

Stalevski, M., Ricci, C., Ueda, Y., et al. 2016, MNRAS, 458, 2288

Stetson, P. B. 1987, PASP, 99, 191

Suganuma, M., Yoshii, Y., Kobayashi, Y., et al. 2006, ApJ, 639, 46

Tody, D. 1986, Proc. SPIE, 627, 733

Tody, D. 1993, in ASP Conf. Ser. 52, Astronomical Data Analysis Software and Systems II, ed. R. J. Hanisch, R. J. V. Brissenden, \& J. Barnes (San Francisco, CA: ASP), 173

Urry, C. M., \& Padovani, P. 1995, PASP, 107, 803

Vazquez, B., Galianni, P., Richmond, M., et al. 2015, ApJ, 801, 127

Wright, E. L., Eisenhardt, P. R. M., Mainzer, A. K., et al. 2010, AJ, 140, 1868

Xie, Y., Li, A., \& Hao, L. 2017, ApJS, 228, 6

Zu, Y., Kochanek, C. S., Kozłowski, S., \& Udalski, A. 2013, ApJ, 765, 106

Zu, Y., Kochanek, C. S., \& Peterson, B. M. 2011, ApJ, 735, 80 